\title{
Atomistic Modeling of Gas Adsorption in Nanocarbons
}

\author{
G. Zollo and F. Gala \\ Department of Fundamental and Applied Science for Engineering-Physics Section, University of Rome "La Sapienza", \\ via A. Scarpa 14-16, 00161 Rome, Italy
}

Correspondence should be addressed to G. Zollo, giuseppe.zollo@uniroma1.it

Received 11 July 2012; Revised 27 September 2012; Accepted 28 September 2012

Academic Editor: Jinquan Wei

Copyright (C) 2012 G. Zollo and F. Gala. This is an open access article distributed under the Creative Commons Attribution License, which permits unrestricted use, distribution, and reproduction in any medium, provided the original work is properly cited.

\begin{abstract}
Carbon nanostructures are currently under investigation as possible ideal media for gas storage and mesoporous materials for gas sensors. The recent scientific literature concerning gas adsorption in nanocarbons, however, is affected by a significant variation in the experimental data, mainly due to the different characteristics of the investigated samples arising from the variety of the synthesis techniques used and their reproducibility. Atomistic simulations have turned out to be sometimes crucial to study the properties of these systems in order to support the experiments, to indicate the physical limits inherent in the investigated structures, and to suggest possible new routes for application purposes. In consideration of the extent of the theme, we have chosen to treat in this paper the results obtained within some of the most popular atomistic theoretical frameworks without any purpose of completeness. A significant part of this paper is dedicated to the hydrogen adsorption on C-based nanostructures for its obvious importance and the exceptional efforts devoted to it by the scientific community.
\end{abstract}

\section{Introduction}

The discovery of novel carbon nanostructures (CNSs) has caused many expectations for their potential impact in gas adsorption, storage, and sensing thanks to their large surface/volume ratio. Gas adsorption is particularly focused on hydrogen for clean energy sources and "small-scale" devices for fuel cells, involving either the hydrocarbon reforming or the hydrogen storage, are currently under study.

The amount of hydrogen storage in solid substrates for commercial use has been estimated to about $9 \mathrm{wt} \%$ for the year 2015 by the US Department of Energy (DOE) [1] but none of the major storage media has reached this value so far.

Gas storage is important also for many other technological applications: helium and nitrogen, for example, have many applications in metallurgic industry.

Nanostructured media are also required for high sensitivity monitoring of chemical species in many fields, from medical to environmental applications. Monitoring of nitrogen dioxide and carbon mono- and dioxide, for instance, is important for the environment while the detection of ammonia $\left(\mathrm{NH}_{3}\right)[2,3]$ and hydrogen sulphide $\left(\mathrm{H}_{2} \mathrm{~S}\right)[4]$ is compulsory in industrial, medical, and living environments.
Many experiments made on gas adsorption in CNSs have evidenced, however, controversial results with partial understanding of the processes involved [5].

The adsorption processes in CNSs can be, in fact, quite tricky because chemisorption and physisorption phenomena may coexist and, moreover, weak interactions are highly sensitive to temperature, pressure, humidity, and so forth, that may vary between different experiments [6].

Another source of uncertainty is that CNS samples are often impure as uncontrolled phenomena and contamination may occur during synthesis [7] resulting in a variety of carbon structures.

All these aspects are strong motivations for reliable atomistic modelling because the understanding of the adsorption/desorption processes is intimately related to the character and the strength of the atomistic interactions. The methods chosen to model such systems, however, may vary greatly depending on the level of accuracy, on the number of particles treated, and on the specific system considered.

The paper is organised as follows: in the next section we give an overview on the recent literature on gas adsorption CNSs approached by the main atomistic theoretical schemes that are introduced in Section 3; in the following three sections, results on gas physisorption and chemisorption in 
NCSs by atomistic modelling are detailed and revised critically with some emphasis on hydrogen and methane due to their importance in technology. Finally some concluding remarks emerging from the general scenario are drawn for the different cases treated.

\section{Overview of Gas Adsorption in Nanocarbons}

Carbon materials exhibit quite different adsorption properties depending on the valence states. Moreover, stable carbon phases may coexist in amorphous carbon where "graphitelike" or "diamond-like" short range order may occur. The other metastable carbon allotropes, such as graphene, fullerenes, carbon nanotubes, carbon nanohorns, and so forth, constitute the backbone of a novel carbon-based chemistry and nanotechnology and exhibit different adsorption properties; in the following of this paragraph, the recent literature on atomistic simulations of gas adsorption is briefly introduced for various allotropes.

2.1. Graphene and Activated Carbons. From graphene, that may be considered a CNS by itself $[8,9]$, several other CNSs can be processed such as armchair semiconducting or zigzag metallic graphene nanoribbons (GNRs), obtained by standard lithography [10], or graphite nanofibers (GNFs). Nanostructured graphite, either hydrogenated or not, can be synthesized by ball milling in a controlled atmosphere, and also activated carbons, consisting of a multitude of stacks of disordered graphene planes of various sizes, are obtained from graphene by steam or chemical agents processing.

Systems for hydrogen storage in graphene-based NSs have been studied concerning both physisorption and chemisorption $[11,12]$ revealing that doping or defects affect the storage capacity as found, for instance, in Li doped graphene [13].

Thanks to their metallic behavior, graphene layers have been widely studied also for gas sensing applications of various gas species $\left(\mathrm{NO}_{2}, \mathrm{H}_{2} \mathrm{O}, \mathrm{NH}_{3}, \mathrm{O}_{2}, \mathrm{CO}, \mathrm{N}_{2}\right.$ and $\left.\mathrm{B}_{2}\right)$ exploiting the charge carrier density change induced by the adsorption [14-16]; some examples of graphene-based "nanodevices" for $\mathrm{pH}$ sensors [17] and biosensors [18] can also be found in the literature.

2.2. Fullerenes. Fullerenes and related structures are usually considered as ideal adsorbents. $\mathrm{C}_{60}$ (bucky-ball) can stably host atoms of the appropriate size, either inside or outside its spherical structure. Hexagonal lattices of $\mathrm{C}_{60}$ molecules can be deposited on a substrate in monolayered or multilayered films while, at low temperatures, cubic $\mathrm{C}_{60}$ lattices (fullerite) are favored; since these fullerene lattices have large lattice constants (hundreds of nanometers), they are fairly appealing open structures for gas storage [19, 20]; thus different adsorption sites in, f.i., hexagonal $\mathrm{C}_{60}$ monolayers have been studied.

Charged fullerenes have been explored for helium adsorption [21] or as $\mathrm{H}_{2}$ storage media as well [22]; moreover it has been shown that "bucky-balls" can also easily bind other gas molecules thanks to their polar properties.
Doping of fullerenes may improve the adsorption of molecular hydrogen and many examples can be found involving light elements, such as fluorine, nitrogen, boron [23], alkali [24-26] and transition metals (TMs) [27-30] and silicon [31].

2.3. Carbon Nanotubes. Single walled carbon nanotubes (SWCNTs) [32, 33] are single graphene sheets folded to form a cylindrical shape in various ways (chirality) resulting in semiconducting or metallic behavior [7].

Gas adsorption occurs in carbon nanotube (CNT) arrays both inside and outside the tubes and hydrogen storage has been intensively studied using classical models [3437], quantum mechanics [38-40], and hybrid models [41] showing that $\mathrm{H}_{2}$ molecules are weakly bound on carbon nanotubes even though $\mathrm{H}$ chemisorption may also occur [42]. Similarly to fullerenes, doping of CNTs with metal species such as $\mathrm{Ti}$ [28], Li, Pt, and Pd [43-45] can improve the adsorption.

Functionalized CNTs are predicted to be suitable for sensing of hydrocarbons [46-51] that can be employed also as hydrogen sources in reforming processes [52].

Defects insertion, structural deformation, or doping are also employed to improve the binding of low adsorption gaseous species on bare CNTs [53]. B or N doped CNTs exhibit nice adsorption features for $\mathrm{H}_{2} \mathrm{O}$ and $\mathrm{CO}$ molecules [54] while TMs doped zigzag and armchair SWCNT have been studied for the detection of $\mathrm{N}_{2}, \mathrm{O}_{2}, \mathrm{H}_{2} \mathrm{O}, \mathrm{CO}, \mathrm{NO}$, $\mathrm{NH}_{3}, \mathrm{NO}_{2}, \mathrm{CO}_{2}$, and $\mathrm{H}_{2} \mathrm{~S}[44,48,55]$. Concerning sensing, however, metal doped SWCNTs are still problematic because their transport properties are weakly affected by the adsorbed molecules [56]. CNT bundles have been studied also for the storage of noble gases such as $\mathrm{He}, \mathrm{Ne}, \mathrm{Ar}$ and $\mathrm{Xe}$ and $\mathrm{N}_{2}$ $[36,42,47,57,58]$.

2.4. Other CNSs. Single walled carbon nanohorns (SWC$\mathrm{NHs}$ ) are conical-shaped graphene sheets that tend to form spherical aggregates with "internanohorn" and "intrananohorn" accessible pores; hydrogen and nitrogen adsorption in such structures have been studied both experimentally and theoretically $[59,60]$.

During the synthesis it may happen that one or several fullerenes get stacked in the internal cavity of a nanotube [61]. Such "peapod" structures are ideal gas "nanocontainers" with enhanced binding properties [62].

\section{Theoretical Methods}

Various atomistic simulation schemes are currently employed to model gas adsorption in CNSs. Of course, chemisorption and bonding events must be approached by quantum theory and CNSs as gas sensors, which require an accurate description of the chemical interactions and the electronic properties, are approached by quantum chemistry techniques $[63,64]$ or ab-initio calculations based on the Density Functional Theory (DFT) $[65,66]$. These schemes are also used to study the equilibrium configurations of physisorbed molecules in CNSs. 
Collective studies on gas adsorption are usually studied with the Metropolis scheme $[67,68]$ in various statistical ensambles.

Other models based on the continuum theory of fluids are also used to model the gas adsorption experiments in carbon porous materials [69-72].

In the next section we introduce briefly the above-listed, not exhaustively, theoretical schemes evidencing their limits of validity and accuracy levels.

3.1. Density Functional Theory ab-Initio Calculations. DFT $a b$-initio calculations $[73,74]$ are efficient tools to study atomistic systems and processes $[75,76]$. According to DFT, the total energy of a system of ions and valence electrons is a functional of the electron density $n(\mathbf{r})$ :

$$
E[n(\mathbf{r})]=F_{\mathrm{HK}}[n(\mathbf{r})]+\int v_{\mathrm{ext}}(\mathbf{r}) n(\mathbf{r}) d \mathbf{r},
$$

where $v_{\text {ext }}(\mathbf{r})$ is the ionic potential. The universal HohenbergKohn functional is $F_{\mathrm{HK}}[n(\mathbf{r})]=T[n(\mathbf{r})]+V_{e_{-e}}[n(\mathbf{r})]$ where $T[n(\mathbf{r})]$ and $V_{e-e}[n(\mathbf{r})]$ are, respectively, the electron kinetic energy and the electron-electron interaction energy; $V_{e-e}[n(\mathbf{r})]$ contains the Coulomb and exchange-correlation energy $V_{e-e}[n(\mathbf{r})]=V_{\mathrm{H}}[n(\mathbf{r})]+V_{\mathrm{XC}}[n(\mathbf{r})]$. The total energy is variational with respect to the electron density and the ground state is obtained self-consistently [75-77].

The key factors affecting the accuracy of the DFT calculations are the pseudopotentials, used to replace the ionic potential (for computational reasons) [78-83], and the scheme adopted to approximate the exchange-correlation potential $V_{\mathrm{XC}}[n(\mathbf{r})]$ that is unknown a prior; the most popular schemes are the local density (LDA) $[73,77]$ and the generalized gradient (GGA) approximations $[84,85]$ and some of them are very accurate such as PBE, B3LYP, and so forth. [65, 84-87]. Generally speaking, LDA and GGA are robust for chemisorption but inaccurate for long-range interactions even though recent studies have shown that the LDA results are surprisingly accurate in many cases [88].

\subsection{Hartree-Fock Based Quantum Chemistry Techniques.}

Various strategies are used to include the electron correlation in Hartree-Fock (HF) based calculations [63, 64, 89-92]; in the Configuration Interaction (CI) scheme, the HF groundstate wavefunction is replaced by a linear combination of ground and excited states obtained by populating virtual molecular orbitals (MOs). CI is very accurate but limited to very small systems for computational reasons. Various CI schemes are used, namely, CIS, CISD amd SF-CISD including, respectively, single, single and double (CIS) and spin flips excited states [63, 64, 90, 92].

In the Möller-Plesset method (MP) the correlation potential $V$ is treated as a perturbation of the HF Hamiltonian $\mathrm{H}=\mathrm{H}^{(0)}+\lambda V$ and, for a system of $n$ electrons, $N$ nuclei and $m$ occupied states, it is formally defined as

$$
V=\sum_{i=1}^{m} \sum_{j>i}^{m} \frac{1}{r_{i j}}-\sum_{i=1}^{m} \sum_{j>i}^{m}\left(J_{i j}-\frac{K_{i j}}{2}\right)
$$

with $J_{i j}, K_{i j}$ being the usual HF Coulomb and exchange integrals.
The exact wavefunction is obtained by solving the secular equation where both wavefunctions and the eigenvalues are expanded in Taylor series of the perturbation parameter $\lambda$; the $q$-order of the wavefunction expansion in terms of a complete set of the HF eigenfunctions is denoted as $\mathrm{MP}_{q}$. MP2 is enough efficient but the correlation energy can be severely underestimated while MP4 is quite accurate, but limited to small systems due to computational limits.

Beyond MPq theory, multiconfiguration states are used instead of single determinants (MCSCF: multiconfiguration self-consistent field) with various "multireference" perturbation schemes such as CASTP2D [63, 64, 90, 92].

The Coupled Cluster (CC) theory [63, 93] is virtually equivalent to a full-CI approach because the wavefunction is represented as

$$
\Psi=e^{T} \Psi_{\mathrm{HF}},
$$

where $T=T_{1}+T_{2}+T_{3}+T_{4}+\cdots$ is the "cluster operator" that formally includes all the possible excited states, $T_{i}$ being the state with $i$ excitations of the HF ground state [90]. Among the different CC schemes encountered, one of the most popular is the $\operatorname{CCSD}(\mathrm{T})$ that includes also a singles/triples coupling term [90].

3.3. Monte-Carlo Sampling Techniques in the Grand-Canonical Ensamble. The Metropolis algorithm [67] allows the Monte Carlo sampling of a $N$-particles statistical ensamble such as the Grand Canonical one that is suitable to study gas adsorption. Many particles are required in this scheme and thus reliable classical atomistic interaction potentials must be used [68]. A physical quantity is measured by calculating it statistically over the ensamble that is generated by using acceptance rules that depend on the energy and the particles number. Hence, the pressure dependence of the equilibrium gas density in CNSs can be calculated. The above described Grand Canonical Monte Carlo (GCMC) method is suitable for large scale gas adsorption studies provided that chemical events, such as bonding, reactions, and so forth, are excluded; the key factor affecting the reliability of GCMC simulation is the accuracy of the interaction potential and still nowadays, the simple Lennard-Jones (LJ) potential (and the ones derived from it) is a popular choice $[36,42,94]$.

Quantum effects are encompassed mainly through the Path Integral Monte Carlo (PIMC) approach where the quantum system is mimicked by a classical polymeric ring system whose equilibrium properties return the statistical properties of the quantum system $[37,95,96]$.

3.4. DFT Nonuniform Fluid Models. In the spirit of DFT, a variational method to find the ground state particles density in fluids was developed with particular emphasis for fluids close to surfaces $[69,70]$. For such systems, the intrinsic free energy functional that must be minimized (e.g., by solving the Euler-Lagrange equations) is made of two terms:

$$
F[\rho(\mathbf{r})]=F_{\mathrm{HS}}[\rho(\mathbf{r})]+\frac{1}{2} \iint u\left(\mathbf{r}, \mathbf{r}^{\prime}\right) \rho(\mathbf{r}) \rho\left(\mathbf{r}^{\prime}\right) d \mathbf{r} d \mathbf{r}^{\prime},
$$

where $F_{\mathrm{HS}}[\rho(\mathbf{r})]$ is the universal "hard-sphere" free energy functional that contains the repulsive energy, and $u\left(\mathbf{r}, \mathbf{r}^{\prime}\right)$ is 
the attractive part of the pairwise interaction potential. As $F_{\mathrm{HS}}[\rho(\mathbf{r})]$ is not known a priori, the Local Density (LDA) or the Smoothed Density Approximations (SDA) can be employed $[71,72]$. In the "non-local density functional theory" (NLDFT), SDA is adopted, the density being smoothed by an appropriate weight function, in order to reproduce the Percus-Yevick description of a homogeneous hard sphere fluid [97]. With this approach, the structural properties and the adsorption isotherms of gases are calculated, and the pore size distribution of the adsorbant can be extrapolated.

\section{Physical and Chemical Adsorption of Gaseous Species in CNSs}

In the previous sections we have emphasized that atomistic modeling of gas adsorption in CNSs should be treated differently depending on the specific phenomena involved, either physical adsorption or chemical bonding.

Therefore, we will treat separately the gas adsorption in CNSs depending whether either physical adsorption phenomena or chemical bonds are involved and, as a consequence, the next two sections will be focused on the physisorption (Section 5) and chemisorption (Section 6) phenomena, respectively.

Sometimes, however, the classification of the studied phenomena in terms of physical or chemical adsorption is quite difficult due to the occurrence of strong polar interactions or weak charge transfer that make uncertain the classification of the case under study; in these cases, the calculation of energetic quantities, such as the activation energy or the adsorption enthalpy, may help to get a clearer scenario because it is expected that physical adsorption exhibits lower adsorption enthalpy values than the ones involved in chemical bonds.

\section{Gas Physical Adsorption in CNSs}

A great deal of the scientific literature over the past twenty years has been devoted to hydrogen physical adsorption in carbon nanomaterials of different allotropic forms due to the potential impact of nanotechnology to solve this challenging problem that is still preventing the hydrogen economy from the success.

Thus we have chosen to dedicate the next subsection to hydrogen storage and to treat the other gaseous species in the following subsections.

5.1. Hydrogen Physical Adsorption. In order to evaluate the hydrogen storage performance of CNSs, one should always refer to the DOE target as the minimum extractable loading for addressing the commercial storage needs.

The typical parameters used to measure the storage are the gravimetric excess (excess hydrogen adsorption):

$$
E_{a}=\frac{m_{\mathrm{H}_{2}}-m_{\mathrm{H}_{2}}^{0}}{m_{\mathrm{H}_{2}}-m_{\mathrm{H}_{2}}^{0}+m_{\mathrm{ads}}} .
$$

$\left(m_{\mathrm{H}_{2}}^{0}, m_{\mathrm{H}_{2}}\right.$, and $m_{\mathrm{ads}}$ being, respectively, the free and the precipitated molecular hydrogen mass and the mass of the

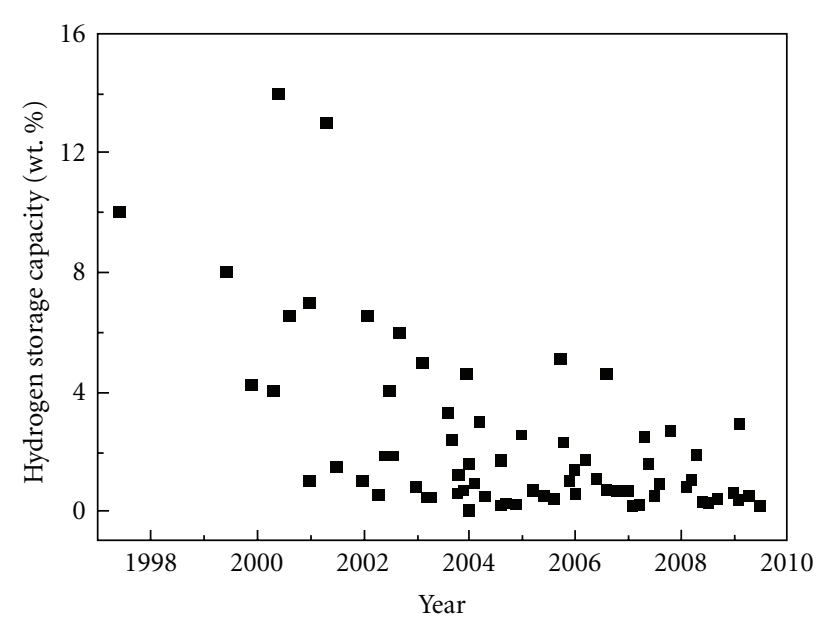

FIGURE 1: CNT hydrogen storage capacities from the literature versus the year of publication. From [40].

adsorbent nanostructured material) and the analogous volumetric excess.

CNSs can be considered as ideal hydrogen adsorption media due to the high surface/volume ratio favouring physisorption with fast adsorption/desorption thermodynamic cycles. The atomistic modelling of such phenomena in nanotubes or fullerenes benefit from their well-known atomic arrangements and thus the possible discrepancies between theory and experiment arise from impurities, samples dishomogeneity, and the limits inherent in the theoretical approach adopted.

On the contrary, complex CNSs, such as activated carbon (AC) or microporous carbon, are particularly challenging because require a great deal of effort to build reliable atomistic models. Thus the literature on atomistic modelling of hydrogen adsorption in CNSs is discussed with reference to the various allotropes considered.

5.1.1. Carbon Nanotubes. Early experiments on CNTs, dating back to the end of the ' $90 \mathrm{~s}$, indicated these CNSs as ideal candidates to fulfill the DOE requirements [98]. Since then, many controversial theoretical and experimental results appeared; recent review papers [99-102] have discussed the experimental data spread (see Figure 1), especially affecting the early measurements, suggesting that it originates from experimental errors and from the samples inhomogeneity and impurity. Nowadays, new purification strategies have been introduced evidencing that hydrogen storage in CNT media may be problematic.

$\mathrm{H}_{2}$ physical adsorption in SWCNT or MWCNT systems has been mainly studied by GCMC and molecular dynamics simulations using simple LJ-derived potentials which have been proven to give realistic results. Apart from early, unconfirmed results [34] supporting the exceptional uptake performance reported in coeval measurements [98], atomistic modelling have evidenced a complicated scenario: $\mathrm{H}_{2}$ uptake can occur either on the external (exohedral) or on the internal surface (endohedral) of a SWCNT where atomic hydrogen is unstable and only molecular hydrogen can exist [39]. 
However the endohedral storage is limited by steric hindrance phenomena that may cause the breakdown of the tube if it is excessive.

The LJ parameters of the carbon-gas interactions are usually obtained from the well known Lorentz-Berthelot rules $\sigma_{\mathrm{gC}}=\left(\sigma_{\mathrm{gg}}+\sigma_{\mathrm{CC}}\right) / 2 ; \varepsilon_{\mathrm{gC}}=\sqrt{\varepsilon_{\mathrm{gg}} \cdot \varepsilon_{\mathrm{CC}}}$ where $\sigma_{\mathrm{gg}}, \varepsilon_{\mathrm{gg}}$ are the LJ gas/gas parameters and $\sigma_{\mathrm{CC}}, \varepsilon_{\mathrm{CC}}$ are the LJ carbon/carbon parameters.

Stan and coworkers $[36,42,58,94]$ have integrated the LJ potential over an ideal CNT surface for different gaseous species that casts in the following potential of a molecule in the vicinity of an ideal CNT:

$$
\begin{aligned}
V(r, R) & =3 \pi \theta \varepsilon \sigma^{2} \\
& \times\left[\frac{21}{32}\left(\frac{\sigma}{R}\right)^{10} f_{11}(x) M_{11}(x)-\left(\frac{\sigma}{R}\right)^{4} f_{5}(x) M_{5}(x)\right],
\end{aligned}
$$

where $r$ is the distance from the cylinder axis, $\theta=0.38 \AA^{-2}$ is the surface density of $\mathrm{C}$ atoms, and $R$ is the radius of the ideal cylinder, $x=r / R(x=R / r)$ if $r<R(R<r)$ and

$$
\begin{aligned}
& M_{n}(x)=\int_{0}^{\pi} \frac{d \phi}{\left(1+x^{2}-2 x \cos \phi\right)^{n / 2}} ; \\
& f_{n}(x)= \begin{cases}0 & r<R \\
\left(\frac{R}{r}\right)^{n} & r>R .\end{cases}
\end{aligned}
$$

This model has been used to calculate the uptake of different gases in CNT bundles showing that, provided that the adsorbate molecule is small enough (as in the hydrogen case) and that the tubes are properly arranged in a honeycomb structure, the amount of hydrogen in the interstitial regions is comparable to the one inside the tubes.

Anyway, the approximations included in this model were quite severe (for instance the gas-gas interactions have been neglected) and these results have been revised by GCMC simulations [103] with the Silvera-Goldman potential [104] for $\mathrm{H}_{2}-\mathrm{H}_{2}$ and the LJ potential for $\mathrm{C}-\mathrm{H}_{2}$; it was shown that the adsorption isotherms decrease as the rope diameter increases because the specific area uptake in the interstitial and endohedral sites is nearly independent on the rope diameter (see Figure 2). These results agree with recent experiments and show that the DOE requirements are not satisfied at room temperature in the $1-10 \mathrm{MPa}$ pressure range, even for an isolated SWCNT. PIMC simulations for $(9,9)$ and $(18,18)$ SWCNT arrays, implemented with the Silvera-Goldman and the Crowell-Brown [105] potentials respectively, for $\mathrm{H}_{2}-\mathrm{H}_{2}$ and $\mathrm{C}-\mathrm{H}_{2}$, have shown that quantum effects lower the GCMC results independently on the CNT chirality [106] and confirm that the previous optimistic experimental results on bare CNTs $[98,107]$ cannot be explained by physisorption.

GCMC simulations have been used also to study how hydrogen physisorption in CNT media is affected by oxidation or by hydrogen chemisorption [108] showing that oxidation should favor the endohedral physical adsorption thus increasing both the volumetric and the gravimetric densities

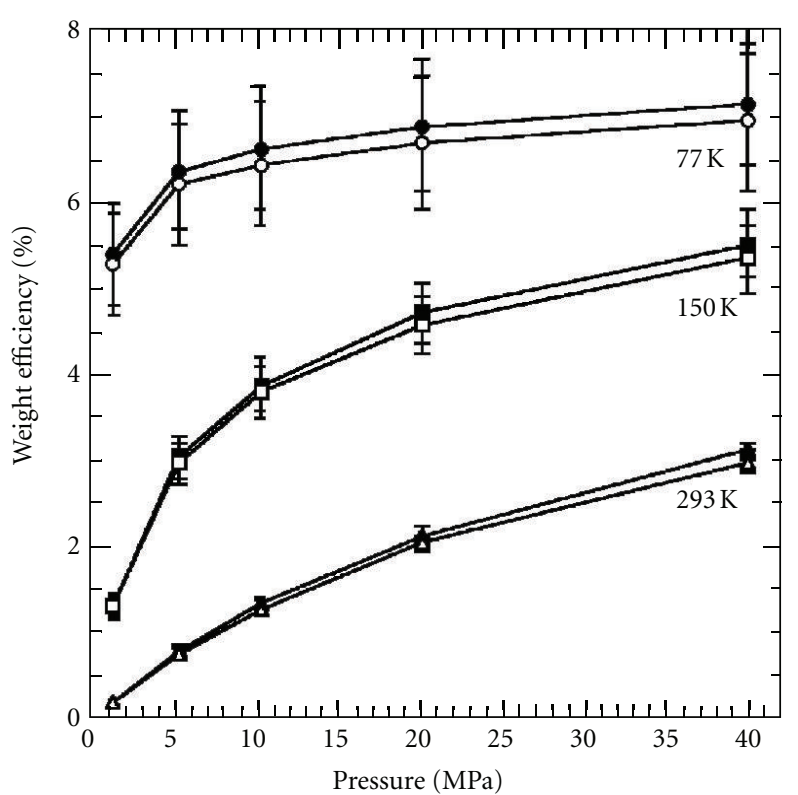

(a)

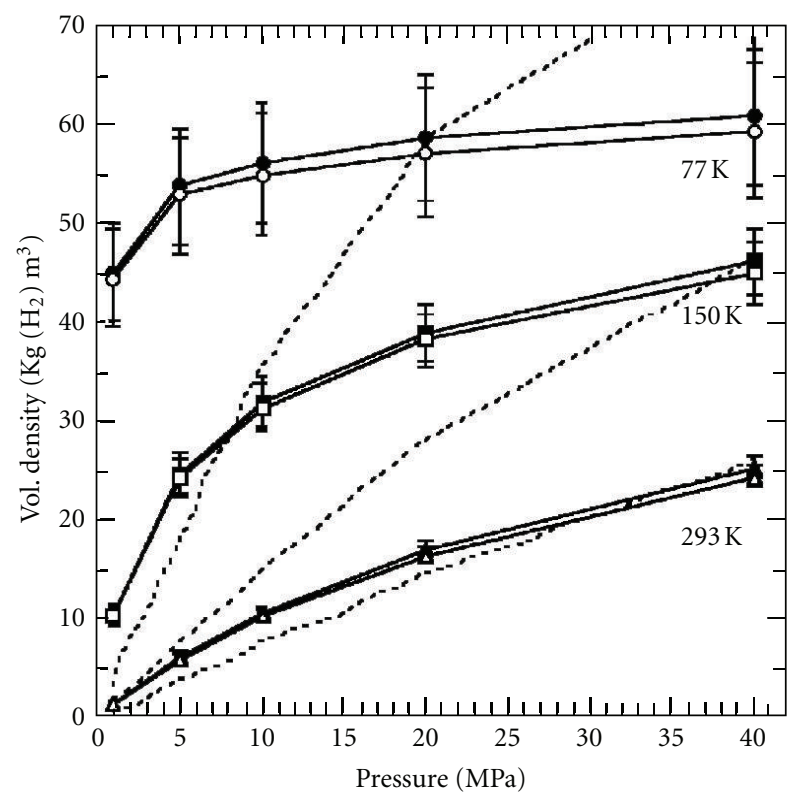

(b)

FIGURE 2: GCMC adsorption isotherms for the $(15,0)$ CNT. (a) gravimetric density; (b) volumetric density for perfect (full symbols) and $10 \%$ hydrogenated (open symbols) CNTs compared to compressed gas data. From [108].

(see Figure 3). The theoretical limits of the hydrogen physical adsorption in SWCNT systems have been discussed by Bhatia and Myers [109] who recast the problem as a delivery one involving storage and release.

The delivery is defined from the adsorption/desorption Langmuir isotherms at different pressures as

$$
D\left(K, P_{1}, P_{2}\right)=\frac{K P_{1} n_{m}}{1+K P_{1}}-\frac{K P_{2} n_{m}}{1+K P_{2}} .
$$




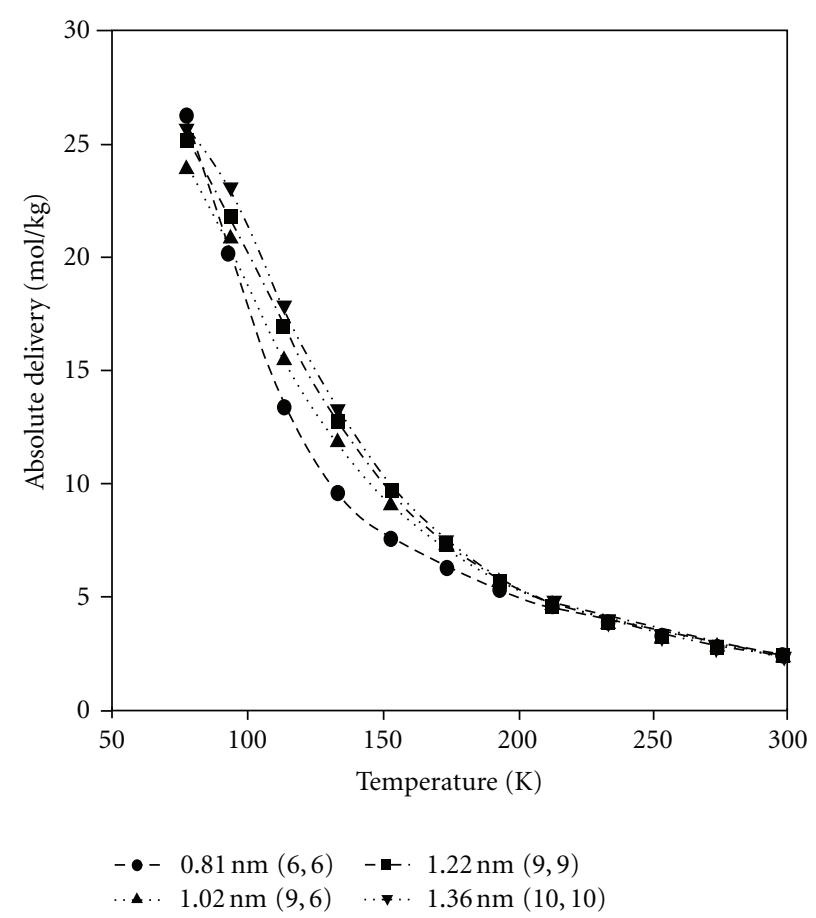

(a)

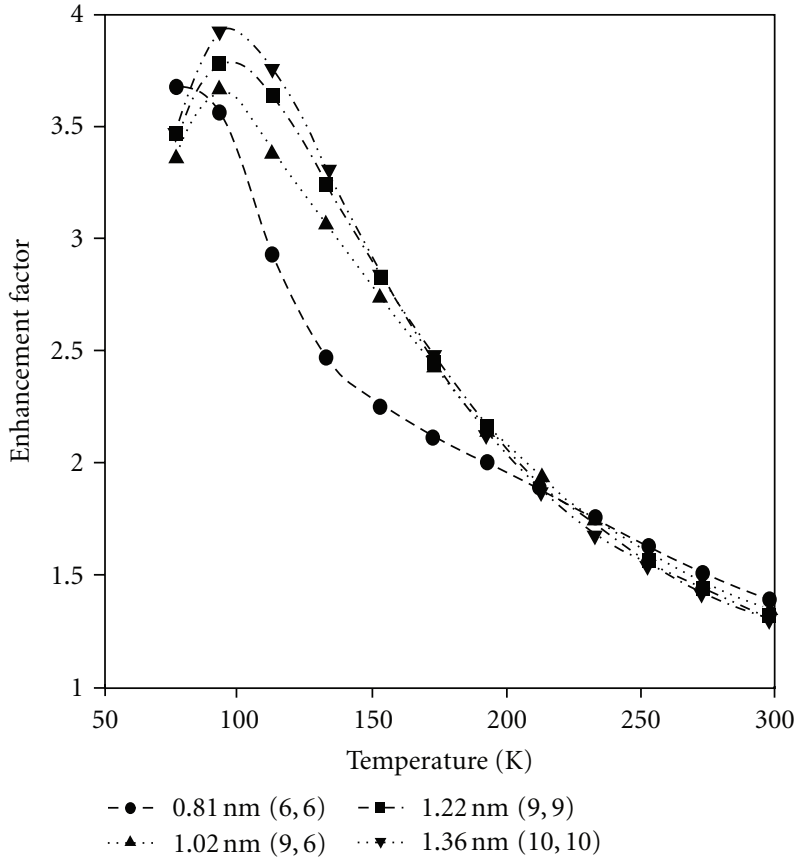

(b)

FIGURE 3: Hydrogen absolute delivery (a) and enhancement factor (b) for CNT arrays with VdW gap of 0.9 nm. From [109].

with $K=1 / P_{0} e^{\Delta S_{0} / R} e^{-\Delta H_{0} / R T} \cdot P_{1}, P_{2}, n_{m}$ are the charge and the discharge pressures and the adsorption capacity and $\Delta \mathrm{H}_{0}, \Delta S_{0}$ are the average heat of adsorption and the entropy change. Using GCMC simulations and thermodynamic arguments, the theoretical maximum delivery has been estimated lower than $4.6 \mathrm{wt} \%$, even for optimal temperature (see Figure 3) given the adsorption heat $\Delta H_{0} \approx 6 \mathrm{~kJ} / \mathrm{mol}$. In this context, the authors evidenced with persuasive arguments that the $\mathrm{H}_{2}$ heat of physisorption on the SWCNT "side-wall" (related to the LJ energy parameter $\varepsilon_{\mathrm{C}-\mathrm{H}_{2}} / k_{B} \approx 31 \mathrm{~K}$ ) makes pure CNTs unfit to satisfy the DOE requirements.

Before drawing conclusive statements, however, it should be emphasized that the LJ energy parameter $\varepsilon_{\mathrm{C}-\mathrm{H}_{2}}$ used in the simulations discussed so far did not include any curvature correction. To correct this discrepancy, the LJ parameters for endohedral and exohedral adsorption have been calculated using quantum chemistry methods: f.i. Guan and coworkers [110] have used MP2 evidencing that the curvature of a $(10,10) \mathrm{CNT}$ makes the endohedral adsorption stronger than the exohedral one.

The difference between the endohedral and exohedral $\mathrm{H}_{2}$ adsorption has been evaluated in the frame of NLDFT for a CNT square lattice arrays showing that the outer adsorption, that depends on the Van der Waals gap (i.e., the "intertubular" distance in a bundle of nanotubes) can be improved [41].

The binding energy of physisorbed $\mathrm{H}_{2}$, calculated by accurate DFT in both zigzag and armchair CNTs, is in the range $0.049 \sim 0.113 \mathrm{eV}$ due to dipolar interactions [48]; these values are slightly improved in nanotube bundles where the adsorption energy for the interstitial and groove sites is larger. Dag and coworkers [111] have tried to clarify the nature and the strength of $\mathrm{H}_{2}$ adsorption on armchair CNTs by using a hybrid model accounting for GGA-DFT short-range interactions and Van der Waals long-range forces [112]. The equilibrium configuration was found at a distance $d_{0}=0.31 \mathrm{~nm}$ with a binding energy of $0.057 \mathrm{eV}$ (almost independently on the tube curvature) that, despite implying the revision of previous results, does not change the whole scenario. Indeed, theoretical calculations have shown that, in order to have good delivery properties with efficient charging/discharging cycles, a system with an adsorption heat of about $15 \mathrm{~kJ} / \mathrm{mol}$ should be considered [109, 113].

Therefore many authors have suggested that CNT adsorbing properties could have been improved by doping with different species, mostly alkali and transition metals. In a Li doped SWCNT, lithium atoms donate the $2 \mathrm{~s}$ valence electrons to the lowest CNT conduction band so that the semiconducting SWCNT becomes metallic. The equilibrium distance from the Li impurity of the physisorbed hydrogen molecule is $d_{0}=0.34 \mathrm{~nm}$ or $d_{0}=0.21 \mathrm{~nm}$ for Li, respectively, internally or externally bonded on the tube [111]. Generally speaking, if the interaction potential and the configuration of the doping alkali metal species are modeled reliably, the hydrogen adsorption results to be enhanced and SWCNT could possibly approach the DOE threshold as shown in Figure 4 [114] where GCMC simulations of Li doped CNT arrays are reported.

High capacity hydrogen storage has been reported in Bdoped or defective nanotubes with Ca impurities [115]; in this case the empty $\mathrm{Ca} 3 d$-orbitals form hybrids with the $\mathrm{H}_{2} \sigma$-orbitals thus enhancing the hydrogen uptake up to 


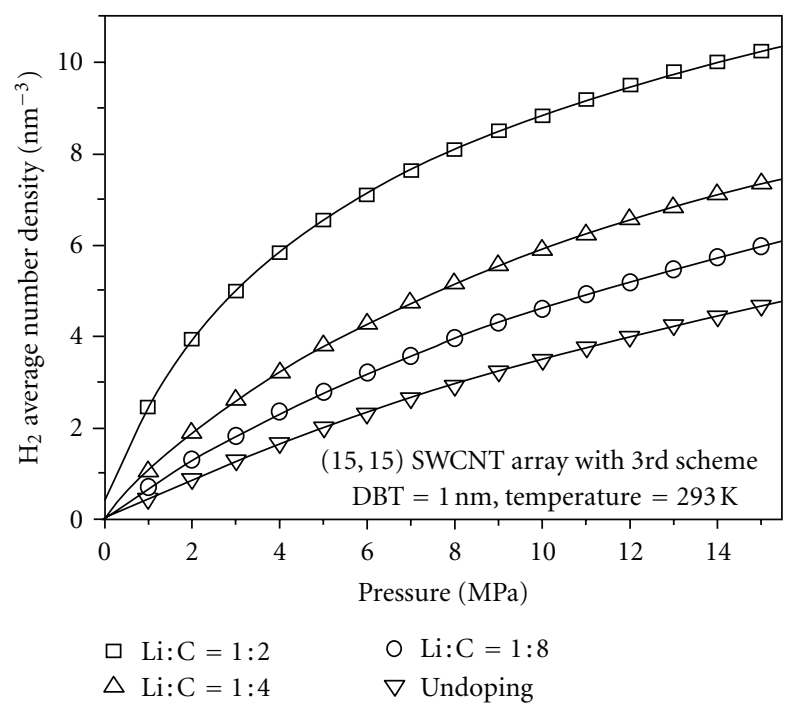

Figure 4: Influence of doping ratio on hydrogen uptake in a $(15,15)$ SWCNT array. From [114].

$5 \mathrm{wt} \%$. In this case, moreover, Ca impurities do not clusterize and remain dispersed on the tube side-wall.

More uncertain is the benefit of this strategy, however, if one considers the whole amount of adsorbed gas and the delivery properties of real samples due to their inherent inhomogeneity and complexity.

Chen and coworkers [43] tried to improve the uptake in a peapod structure obtained by encapsulating a fullerene molecule inside a Li-doped SWCNT; in this case a complex charge transfer process occurs favoring Li charging and its strong bonding to the CNT surface that results in a noticeable increase of $\mathrm{H}_{2}$ binding.

5.1.2. Activated and Microporous Carbons. In the case of activated (AC) and microporous carbon (MPC) as gas storage media, a severe bottleneck for theoretical predictions is the definition of reliable atomistic models for such disordered materials. Also these materials, however, suffer from the limiting factor of the $\mathrm{C}-\mathrm{H}_{2}$ interaction properties that make unlikely large amount of $\mathrm{H}_{2}$ storage by physisorption.

Several potential functions, such as the Mattera, the Taseli [116], and the Steele 10-4 potential [117] have been employed to treat the graphene- $\mathrm{H}_{2}$ interactions in the contexts of the "slit-pore" model where pores are delimited by graphene planes [109]; most of these studies predict similar values of the adsorption heat and the excess gravimetric percentage that is below the DOE requirements at the operating pressure and temperature. In contrast, about $23 \mathrm{wt} \%$ has been obtained by extrapolation at very high pressure conditions where it has been claimed that the hydrogen density exceeds the liquid hydrogen one [118]; anyway this idea has been confuted by molecular dynamics simulations at $77 \mathrm{~K}$ showing also that oxygenation, differently from the CNT case, does not improve the uptake [119].

Recently, well-founded atomistic models of ACs and MPCs have been obtained using the Hybrid Reverse Monte
Carlo (HRMC) scheme $[120,121]$ starting from an initial realistic "slit-like" pore configuration obtained from experimental data on the pore wall thickness and size distribution (PSD).

The HRMC algorithm has been applied on the basis of the following acceptance criterion:

$$
\mathrm{P}_{\mathrm{acc}}=\min \left\{1, \exp \left(-\left(\frac{\Delta \chi^{2}}{2}+\frac{\Delta E^{2}}{k_{B} T}\right)\right)\right\},
$$

with

$$
\chi^{2}=\sum_{i=1}^{N} \frac{\left(\mathrm{g}_{s}\left(r_{i}\right)-\mathrm{g}_{\exp }\left(r_{i}\right)\right)^{2}}{\sigma_{i}^{2}}
$$

where $N, \mathrm{~g}_{s}(r), \mathrm{g}_{\exp }(r), \sigma_{i}$ are, respectively, the number of experimental points, the simulated and the experimental radial distribution functions and, lastly, the errors inherent the experimental data (treated as adjustable parameters).

The AC atomistic model is finally obtained (as shown in Figure 5) by simulated annealing in multiple canonical ensambles with gradually decreasing the temperature and parameters $\sigma_{i}$ in order to minimize the energy and $\chi^{2}$ contextually [120]; an environmentally dependent interaction potential (EDIP) [122] or a reactive empirical bond order potential [123] can be used to this aim. On this basis, GCMC simulations with the Feynman-Hibbs $(\mathrm{FH})$ correction for the quantum dispersion effect $[96,124]$ have been performed at cryogenic temperatures [125]; the FH interaction potential is

$$
\begin{aligned}
U_{\mathrm{FH}}^{X-\mathrm{H}_{2}}(r)= & U_{\mathrm{LJ}}^{X-\mathrm{H}_{2}}(r) \\
& +\left(\frac{\beta \hbar^{2}}{24 \mu}\right)\left(\frac{2}{r} \frac{\partial U_{L J}^{X-\mathrm{H}_{2}}(r)}{\partial r}+\frac{\partial^{2} U_{L J}^{X-\mathrm{H}_{2}}(r)}{\partial r^{2}}\right),
\end{aligned}
$$

where $X=\mathrm{C}, \mathrm{H}_{2}, \mu=m_{X} m_{\mathrm{H}_{2}} /\left(m_{X}+m_{\mathrm{H}_{2}}\right), U_{\mathrm{LJ}}^{X-\mathrm{H}_{2}}(r)$ is the classical LJ potential and the $\mathrm{C}-\mathrm{H}_{2}$ parameters are defined using the Lorentz-Berthelot rule.

The energy parameter of a curved surface has been obtained by correcting the one of a flat surface with the factors $\mathrm{C}_{\varepsilon}=1.134, \mathrm{C}_{\varepsilon}{ }^{2}$ for the surface-fluid and the surfacesurface interactions, respectively. Then the effective FH interaction potential of $\mathrm{H}_{2}$ with an immobile planar carbon wall is calculated and used in a GCMC context where the $\mathrm{H}_{2}-\mathrm{H}_{2}$ interactions have been treated with the Levesque parameters [126]; the C-C interactions have been treated by either the Frankland-Brenner [127] or the Wang et al. [128] parameters obtaining good results while the Steele parameters [129] underestimate the adsorption. On this basis, reliable RT isotherms for ACs and MPCs have been obtained using new LJ parameters with an enhanced well depth (about $\varepsilon_{\mathrm{C}-\mathrm{C}}^{\text {flat }}=$ $37.26 \mathrm{~K}$ ) to correct for the increased surface polarizability occurring when $\mathrm{H}_{2}$ molecules approach the carbon surface.

5.1.3. Other Carbonaceous Structures. Other carbon nanomaterials, such as nanostructured graphite, GNFs, fullerenes, nanohorns, and so forth, are frequently found in the 


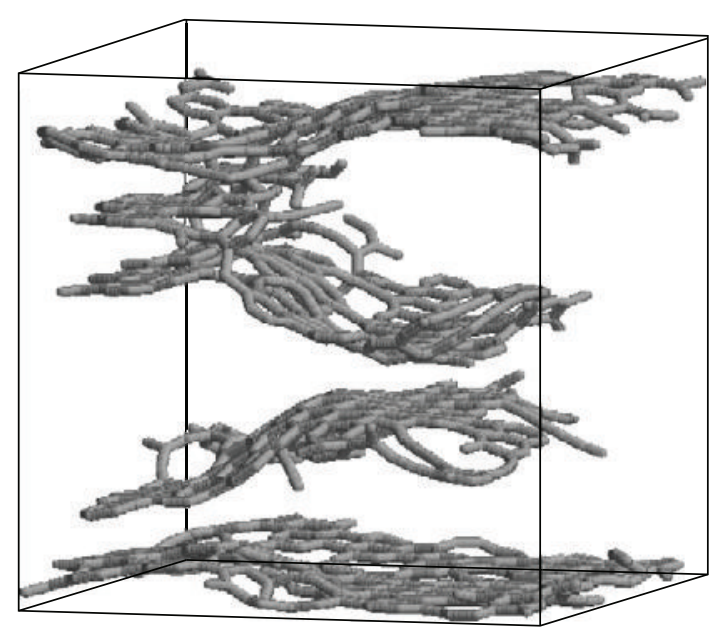

(a)

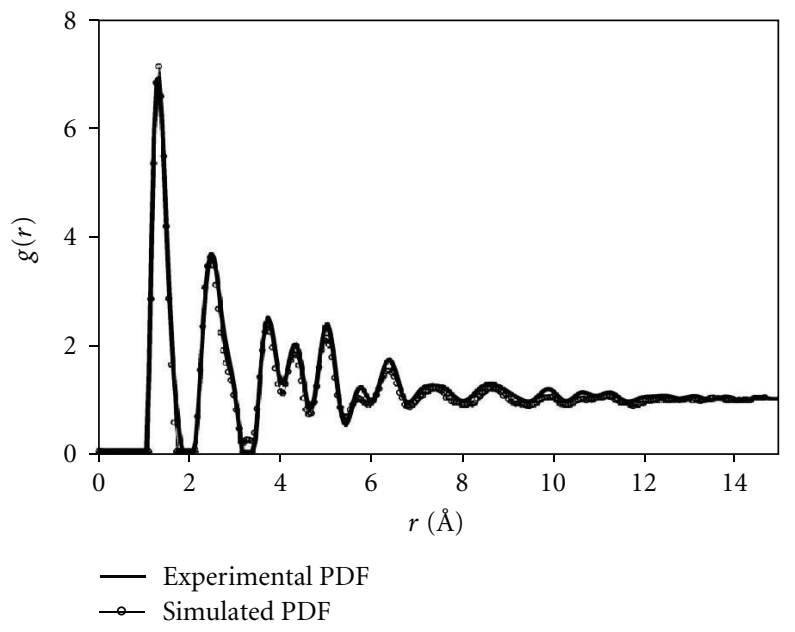

(b)

FIGURE 5: Active carbon reliable configuration (1166 carbon atoms) (a). Comparison between the experimental and simulated pair distribution functions (PDFs) (b). From [125].

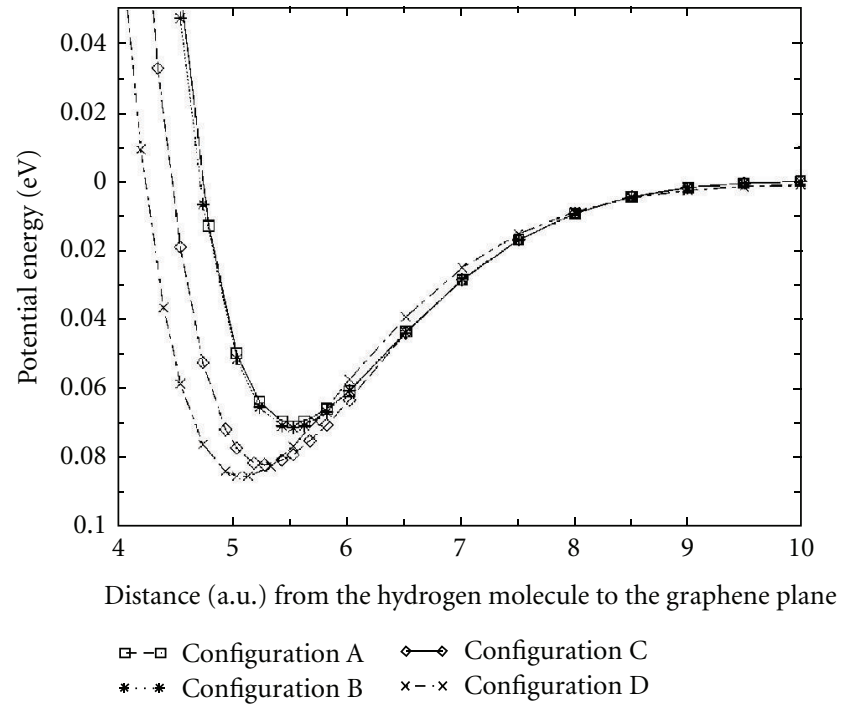

Figure 6: $\mathrm{H}_{2}$ potential energy in the vicinity of a graphene layer. The $\mathrm{H}_{2}$ axis is perpendicular (A, B, C) or parallel (D) to the graphene layer. Positions are above a carbon atom (A), a C-C bond centre (B), a hexagon centre (C), (D) From [11].

literature as potential materials for hydrogen adsorption, sometimes combined with alkali metal hydrides where lithium carbide forms after few adsorption/desorption cicles [130].

The interaction of an $\mathrm{H}_{2}$ molecule with a graphene sheet has been studied by LDA-DFT calculations [11] and the energy curves, obtained by varying the molecule orientation, the adsorption site, and the distance, show the typical van der Waals behaviour for physisorption (see Figure 6). Thus hydrogen uptake in GNFs has been simulated with conventional LJ potentials showing that significant adsorption, in any case below $1.5 \mathrm{wt} \%$ at $\mathrm{P}=10 \mathrm{MPa}$ and RT [131], occurs only if the interplanar distance is larger than $0.7 \mathrm{~nm}$. The usage of more accurate potential parameters, fitted on MP2 ab-initio calculations of vibrational energy or on experimental results [116], has demonstrated that, at cryogenic temperature and ambient pressure, the adsorption capacity of GNFs is about $2 \mathrm{wt} \%$. However, MP2 results are affected by long range errors and reliable potential well and equilibrium distance can be obtained only with a large basis set. On the basis of the above predictions, the experimental results reporting adsorption excess data of $10-15 \mathrm{wt} \%$ at $\mathrm{RT}$ and $\mathrm{P}=8 \mathrm{MPa}[132,133]$ are most probably due to chemisorbed contaminants such as oxygen or residual particles of metal catalysts used during the synthesis; this circumstance has evidenced the potential positive role played by contaminants in storage thus driving the researchers to study metal doping also in these systems. Therefore some authors have suggested doping with alkali metals, such as $\mathrm{Li}$ and $\mathrm{K}$, to increase the uptake. Indeed, Zhu and coworkers [134] have found that charge transfer, occurring from the metal atoms to the graphene layer, enhances the hydrogen adsorption at low temperature while it is significantly weakened at higher temperature. In this case Li is slightly more effective than $\mathrm{K}$ because of the higher charge transfer from $\mathrm{Li}$ to graphene ( $0.5 e$ and $0.2 e$ for $\mathrm{Li}$ and $\mathrm{K}$ respectively) with an $\mathrm{H}_{2}$ binding energy almost doubled with respect to graphene. Because the transferred charge remains localized near the metal atom, the uptake enhancement does not hold if $\mathrm{H}_{2}$ and $\mathrm{Li}$ stay on the opposite sides with respect to the graphene layer [13].

Ca doping of zig-zag GNRs, approached by GGA-DFT, has evidenced an $\mathrm{H}_{2}$ gravimetric capacity of $5 \%$ at $0 \mathrm{~K}$, with reduced clustering of the impurities; clustering can be suppressed also in armchair GNRs by B-Ca codoping [135]. $\mathrm{B}$ codoping has been also explored in Li doped graphene to suppress the metallic clustering and to fully exploit the enhanced interaction for $\mathrm{Li}$ atoms with $\mathrm{H}_{2}$ molecules due to van der Waals forces and hybridization [136]. Other attempts to improve storage in graphene include the usage of graphene 


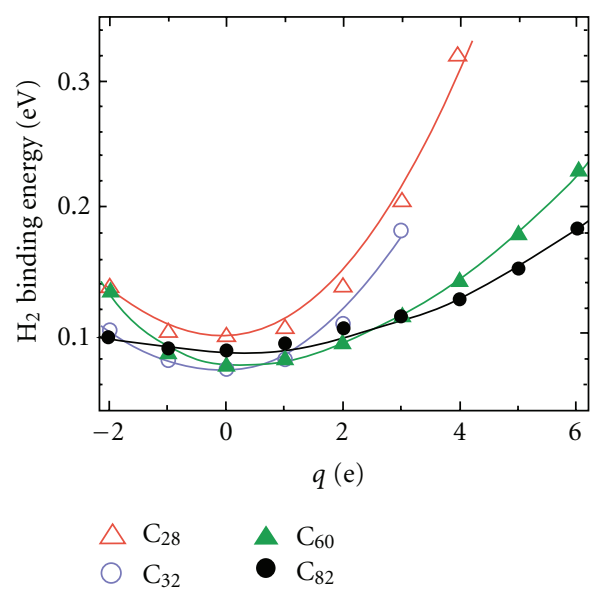

(a)

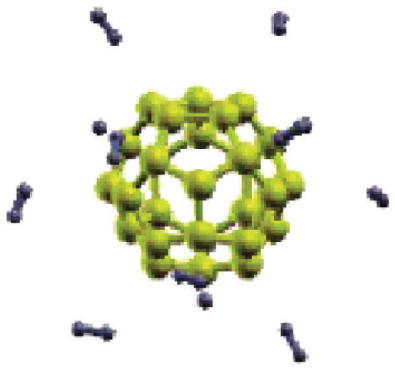

(b)

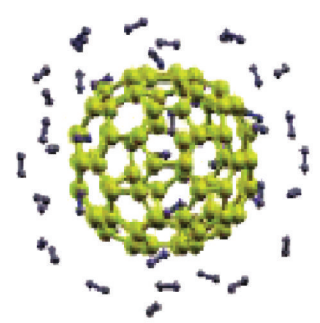

(c)

Figure 7: The binding energy of molecular hydrogen on charged or neutral fullerenes (a). Optimized hydrogen-fullerene complexes of $12 \mathrm{H}_{2}-\mathrm{C}_{28}{ }^{3+}$ (b) and $43 \mathrm{H}_{2}-\mathrm{C}_{82}{ }^{6+}$ (c) with a hydrogen uptake of $6.67 \mathrm{wt} \%$ and $8.04 \mathrm{wt} \%$, respectively. From [22].

layers deposited on metallic substrates [137] showing that $\mathrm{Ni}$ and Pt subtrates behave differently, the first one increasing the covalent bonding on graphene. It should be considered, however, that oxygen adsorption is a competing process that strongly suppresses the hydrogen adsorption in metal doped graphene [138] thus making unlikely the usage of such sysyems for hydrogen storage.

Similarly to other CNSs, fullerenes show low binding energy values (few meV) for molecular hydrogen resulting in poor uptake. Charged fullerenes could be used to improve the uptake performance and $a b$-initio calculations of charged fullerenes $\mathrm{C}_{n}^{q}(-2 \leq q \leq 6,21 \leq n \leq 82)$ have been performed accordingly [26].

As reported in Figure 7(a), the binding energy of a hydrogen molecule adsorbed at the fullerene surface can be increased between two and five times depending on the fullerene charge state whose polarity affects also the $\mathrm{H}_{2}$ orientation. An uptake of $8.04 \mathrm{wt} \%$ has been predicted at best. Figure 8 shows both the electric field generated by the charged fullerene and the hydrogen molecule charge density map obtained under such an electric field, giving, at least classically, a clear insight of the mechanism responsible of the $\mathrm{H}_{2}$ storage.

Charged fullerenes can be produced by encapsulating a metal atom inside the fullerene cage: for instance, by entrapping a La atom inside a fullerene, three electrons are transferred to $C_{n}$. Anyway, in this case, the electric field outside the carbon molecule still does not differ significantly from the neutral case due to charge localization phenomena [22].

Enhanced adsorption on fullerenes can be obtained also with transition metals (TMs) [29]: according to the DewarChatt-Duncanson model [139], the interaction is caused by a charge transfer from the $\mathrm{H}_{2}$ highest occupied molecular orbital (HOMO) to the metal empty $d$-states followed by a back donation from a metal $d$-orbital to the $\mathrm{H}_{2}$ lowest unoccupied orbital (LUMO). $\mathrm{C}_{60}$ decorated with $\mathrm{Ti}$ have been investigated extensively showing a hydrogen adsorption up to $7.5 \mathrm{wt} \%$ depending on doping site: if Ti occupies a hollow site, it strongly binds to the cage and no charge transfer to the hydrogen $\sigma^{*}$ molecular orbitals occurs thus causing hydrogen physisorption; on the contrary, if Ti atoms occupy other sites, at least one $\mathrm{H}_{2}$ molecule dissociates and is bonded to the $\mathrm{Ti}$ atom while the other hydrogen molecules are physisorbed nearby the impurity.

However, Sun and coworkers [140] have found that Ti, similarly to other TMs, tend to agglomerate after the first desorption cycle, thus reducing the hydrogen physisorption and storage. The same authors have demonstrated also that $\mathrm{Li}_{12} \mathrm{C}_{60}$ molecules can bind up to 60 hydrogen molecules resulting in a theoretical gravimetric density of $13 \mathrm{wt} \%$ with a nearly constant binding energy [25]. This is due to the large electron affinity of $\mathrm{C}_{60}$ (about $2.66 \mathrm{eV}$ ) causing the capture of $\mathrm{Li}$ valence electrons that strengthen the bond; then the positively charged Li impurity causes the polarization of the $\mathrm{H}_{2}$ molecules resulting in an increased interaction. Moreover it was also demonstrated that $\mathrm{Li}_{12} \mathrm{C}_{60}$ clustering affects only moderately the hydrogen binding properties.

Alkaly metals doping of $\mathrm{C}_{60}$ have been studied also by $a b$ initio B3LYP/3-21G $(d, p)$ calculations [24]: being positively charged with respect to fullerenes, these impurities can bind up to six ( $\mathrm{Li}$ ) or eight ( $\mathrm{Na}$ and $\mathrm{K}$ ) $\mathrm{H}_{2}$ molecules. By increasing the number of $\mathrm{Na}$ atoms, the average binding energy remains almost constant because each hexagonal ring of the fullerene cage behaves independently showing a highly localized reactivity at the individual rings. $\mathrm{Na}_{8} \mathrm{C}_{60}$ is found to be energetically stable with a theoretical hydrogen gravimetric ratio of $9.5 \mathrm{wt} \%$. DFT calculations of $\mathrm{C}_{60}$ doping with alkali metals of the second group ( $\mathrm{Ca}, \mathrm{Sr}$ ) have evidenced that a strong electric field arises depending on the significant chemical activity of $d$-orbitals in these species [141], unlike Be and Mg: the fullerene $\pi^{*}$-orbital, that is partially occupied by the electrons of the alkali metal $s$-orbital, hybridize with the alkali metal $d$-states thus resulting in net charge transfer that causes the $\mathrm{H}_{2}$ polarization giving a theoretical hydrogen uptake of $8.4 \mathrm{wt} \%$. In Figure 9 spin resolved PDOS (projected DOS, i.e., projected density of states) of a single 


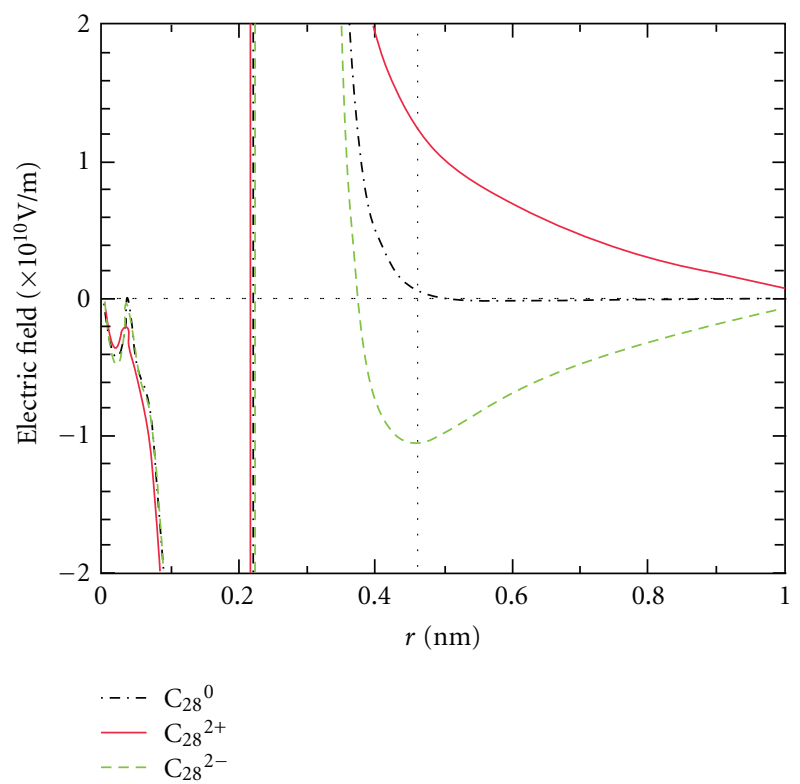

(a)

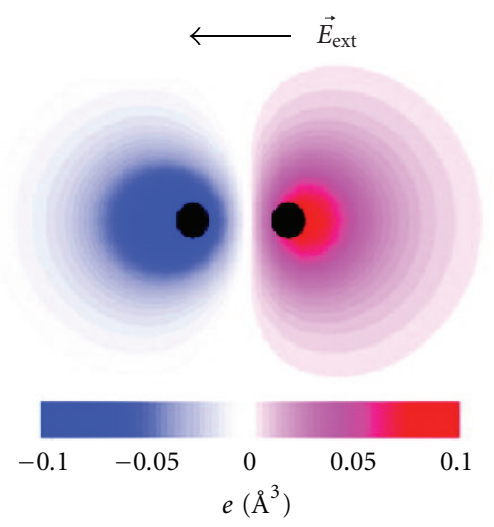

(b)

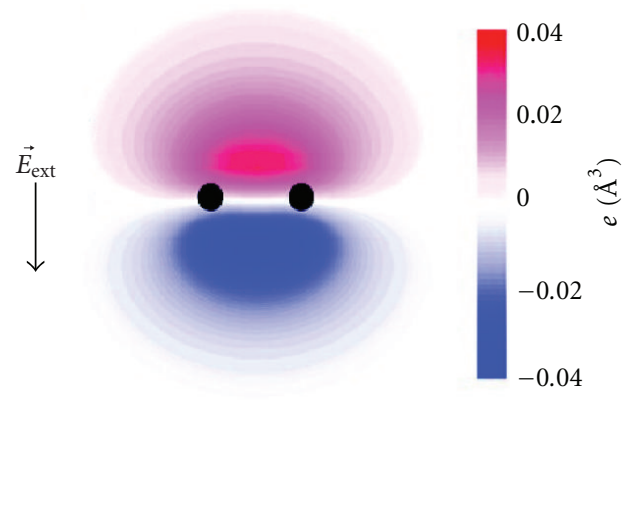

(c)

FIGURE 8: Electricfield associated with neutral and charged fullerenes, $\mathrm{C}_{28}{ }^{q}(q=-2,0,+2)$ at the center of a hydrogen molecule located on top of hexagonal ring (a). Hydrogen charge variations induced by an electric field of $2 \times 10^{10} \mathrm{~V} / \mathrm{m}$ parallel (b) and perpendicular (c) to the molecule axis. From [22].

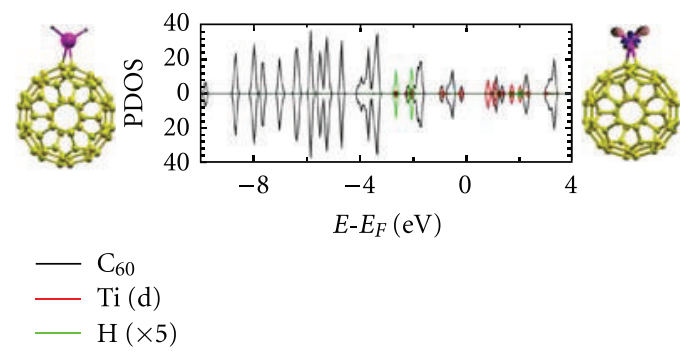

(a)

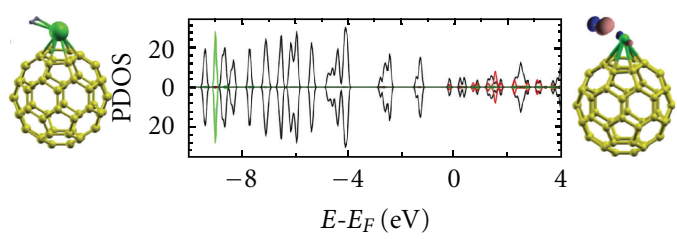

$-\mathrm{C}_{60}$
$-\mathrm{Ca}$
$-\mathrm{H}(\times 5)$

(b)

FIgURE 9: Spin-resolved PDOS (in arbitrary units) of a single hydrogen molecule adsorbed on Ti-coated (a) and Ca-coated (b) fullerenes. For Ti, only the $d$ levels are plotted (the hydrogen states are magnified). The corresponding iso-surfaces of the charge density differences are shown in the right panel. From [26] copyright 2008 by the American Physical Society. 


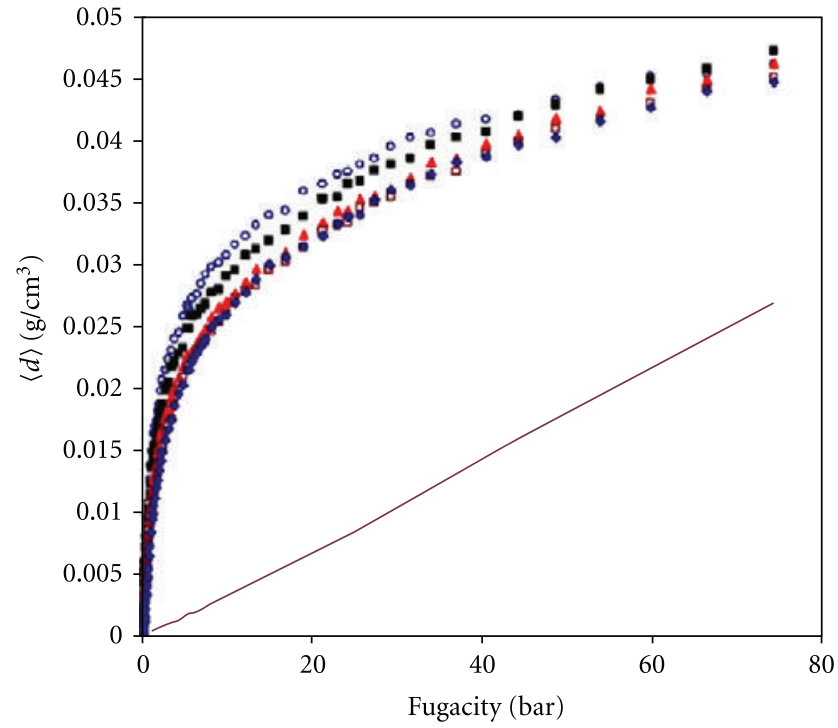

(a) $\mathrm{V}=14.9 \mathrm{~nm}^{3}$.

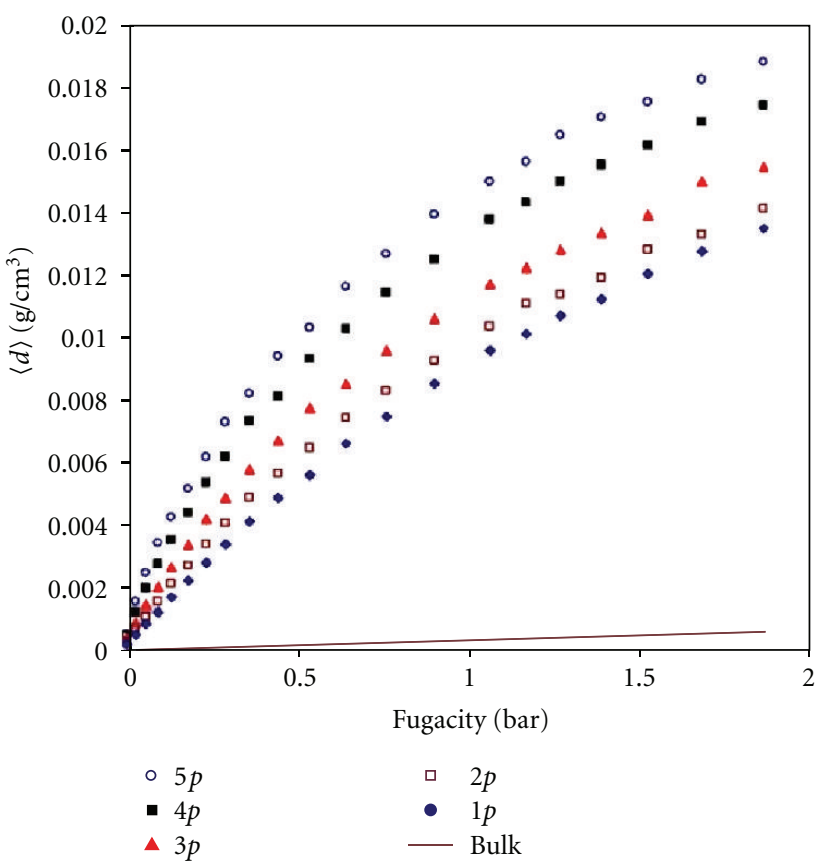

(c) $\mathrm{V}=14.9 \mathrm{~nm}^{3}$.

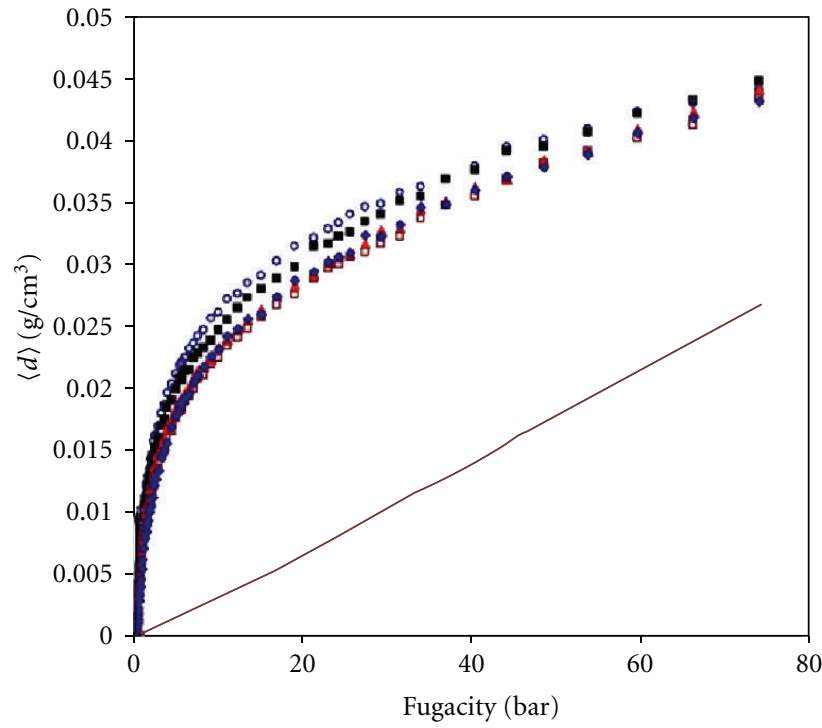

(b) $\mathrm{V}=29.9 \mathrm{~nm}^{3}$.

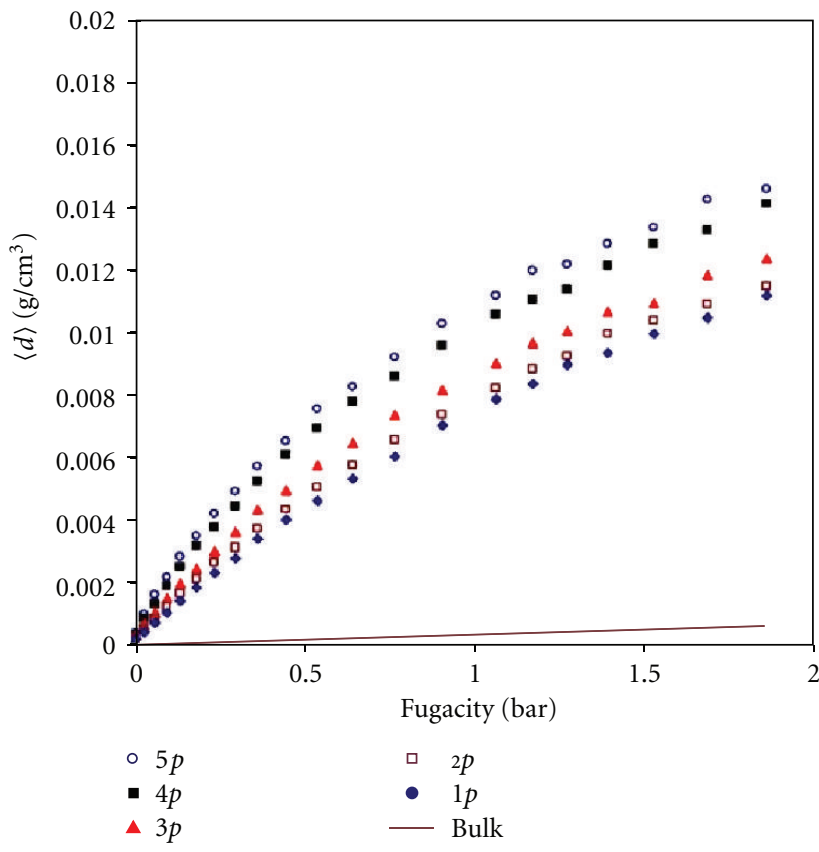

(d) $\mathrm{V}=29.9 \mathrm{~nm}^{3}$.

Figure 10: GCMC calculated isotherms for five cone structures of different sizes: $14.9 \mathrm{~nm}^{3}$ (a and c) and $29.9 \mathrm{~nm}^{3}$ (b and d). Plots (c) and (d) show the low fugacity ( $0-2$ bar) details of (a) and (b) respectively. GCMC results for the bulk hydrogen at $77 \mathrm{~K}$ are shown as lines. From [143].

hydrogen molecule on Ca-coated fullerene evidences that the hydrogen $\sigma$-orbital, located far below the Fermi level, remains unchanged; also the charge density variations, induced by the hydrogen adsorption, suggest that polarization of the $\mathrm{H}_{2}$ occurs near the $\mathrm{Ca}$ atom.

Carbon nanocones (CNCs) have been investigated as possible alternatives to CNTs for hydrogen storage [142]. The adsorption isotherms at $77 \mathrm{~K}$ in CNC structures with different apex angles have been calculated by GCMC simulations [143] where $\mathrm{C}-\mathrm{H}_{2}$ interactions are treated with second order Feynman-Hibbs LJ potentials showing that molecular hydrogen can be confined in the apex region inside the cone, in agreement with recent findings from neutron spectroscopy of $\mathrm{H}_{2}$ in $\mathrm{CNHs}$ [59]. The hydrogen density obtained is reported in Figure 10 as a function of the fugacity. The density behaves differently for the high/low pressure regimes. In any case, the theoretical data demonstrate that the hydrogen uptake is larger in a CNC than in a CNT and this behavior is attributed mainly to the high interaction region close to the apex. Quite recently Ca-decorated carbyne 


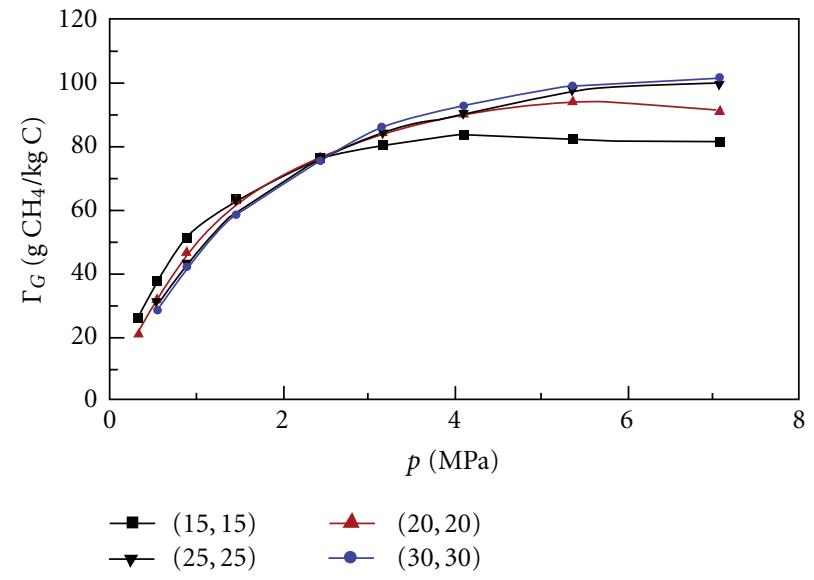

(a)

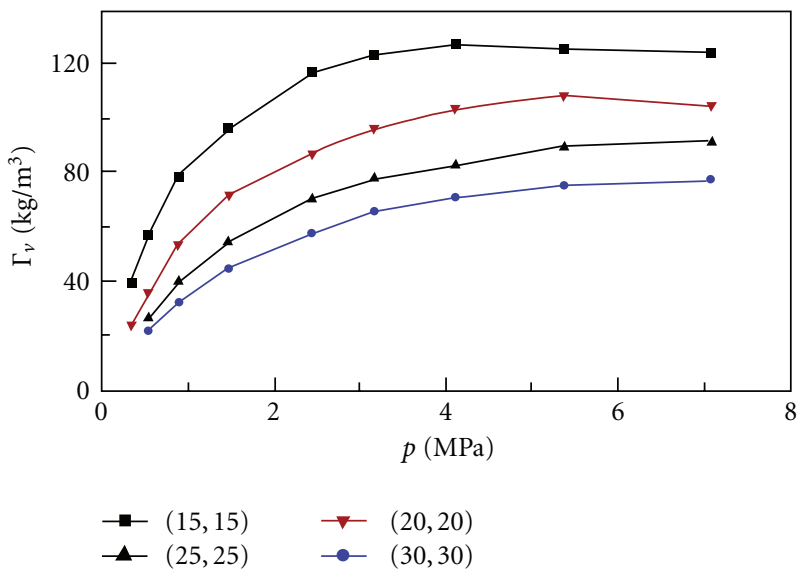

(b)

Figure 11: Endohedral excess adsorption of methane in SWNT arrays. From [145].

networks have been considered in the framework of ab-initio DFT calculations suggesting that this system could benefit of a surface area four times larger than graphene. Each $\mathrm{Ca}$ decorated site has been predicted to adsorb up to 6 hydrogen molecule with a binding energy of $0.2 \mathrm{eV}$ and no clustering was observed in the model [144].

\subsection{Physical Adsorption of Gaseous Species Other than Hydro-} gen. Other gaseous species are considered for adsorption CNSs; among them, noble gases are important case studies because they are used in adsorption experiments at low temperature to measure the CNSs pore size distribution; On the contrary, condensation phenomena occur in these systems that, being studied in the context of the low temperature physics, are beyond the aims of the present review and will be omitted. However modelling of porosimetry experiments concerning carbon microporous and nanoporous media where physical adsorption phenomena do not cause condensation will be explicitly treated.

In the following, moreover, a special emphasis will be devoted to methane adsorption that is attracting a growing interest for alternative automotive energy sources. Methane, in fact, can be efficiently stored in CNSs because of its high physisorption binding energy making it attractive for storage at RT and moderate pressure.

5.2.1. Methane Adsorption. Methane uptake in CNT bundles has been studied by Stan and co-workers for rigid tubes following the same approach adopted for hydrogen (Section 5.1.1) [36, 42]. LJ parameters and Lorentz-Berthelot rules have been employed to calculate the ideal uptake curves $\sigma_{\mathrm{gg}}=\sigma_{\mathrm{gg}}\left(\varepsilon_{\mathrm{gg}}\right)$ (for endohedral and interstitial sites) at low coverage for a threshold gas density and fixed chemical potential and temperature; in spite of the deep potential energy wall $\left(\varepsilon_{\mathrm{gg}}=145 \mathrm{~K}\right)$ of methane in CNSs, low uptake values at moderate pressure were predicted mainly due to the methane molecular size.

GCMC simulations have been performed to calculate the adsorption excess for both the endohedral and interstitial sites of CNTs for different pressure values and van der Waals gaps between the tubes [117, 145, 146] (see Figures 11 and 12). The decreasing behavior of the interstice excess adsorption reveals that the outer uptake saturates while the gas density increases linearly for compression. The usable capacity ratio (UCR), that measures the available fuel upon adsorption/desorption cycles with respect to the available fuel in a storage vessel, is calculated for different loading pressures and van der Waals gaps (see Figure 13).

For each CNT type and loading pressure, it can be found a peculiar arrangement of the CNT array that maximizes the UCR with respect to the volumetric capacity of the compressed natural gas (CNG) (about $200 \mathrm{~V} / \mathrm{V}$ at $20 \mathrm{MPa}$ ) showing that the CNG value is obtained at a much lower pressure in these structures [145]. The potential advantage of carbon tubular "worm-like" structures (CNW: carbon nano worm) over CNTs for methane storage was evidenced by calculating the "Langmuir-type" adsorption isotherms of these structures compared to $(10,10)$ armchair CNTs [147].

As expected, the measured isosteric heat of adsorption is maximum for the most corrugated wormlike tube examined, in accordance with the large methane adsorption excess measured.

Using basically the same method, the isosteric heat of methane adsorption at zero loading in various CNT arrays has been calculated focusing on different uptake sites such as interstitial, surface, groove, "intratubular", and so forth [148]. If allowed, the interstitial adsorption site is the most favorable followed by intratubular, groove, and surface sites.

Hydrogen and methane mixtures (hythane) are also considered for adsorption in CNT arrays, slitlike carbon nanopores, and mesoporous carbons. This is aimed, for instance, to separate hydrogen, and methane in synthetic gas obtained from steam reforming of natural gas or for storage clean fuels on vehicles [149-151].

It has been demonstrated that hythane storage in slitlike pores and CNTs can achieve the volumetric stored energy threshold of $5.4 \mathrm{MJ} / \mathrm{cm}^{3}$ for alternative automotive fuel systems established by the US Freedom Car Partnership. 

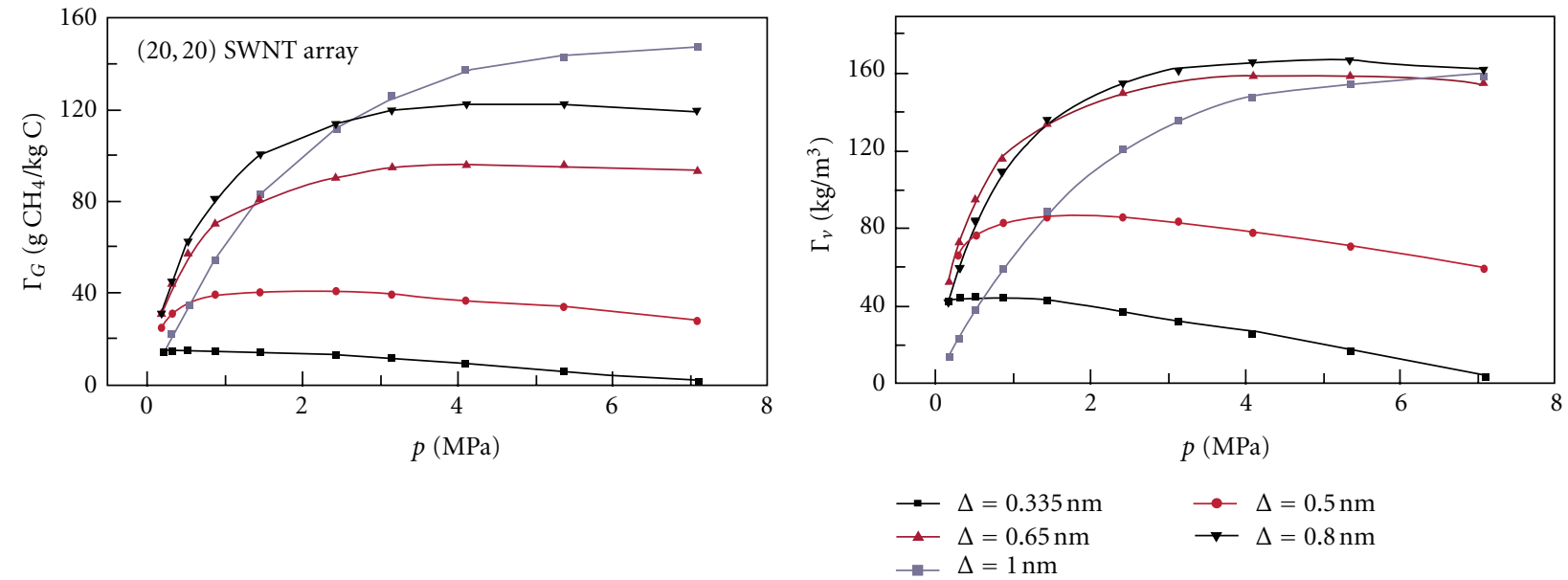

(a)

(b)

FIGURE 12: Adsorption excess of methane in the interstice of the $(20,20)$ SWNT arrays. From [145].

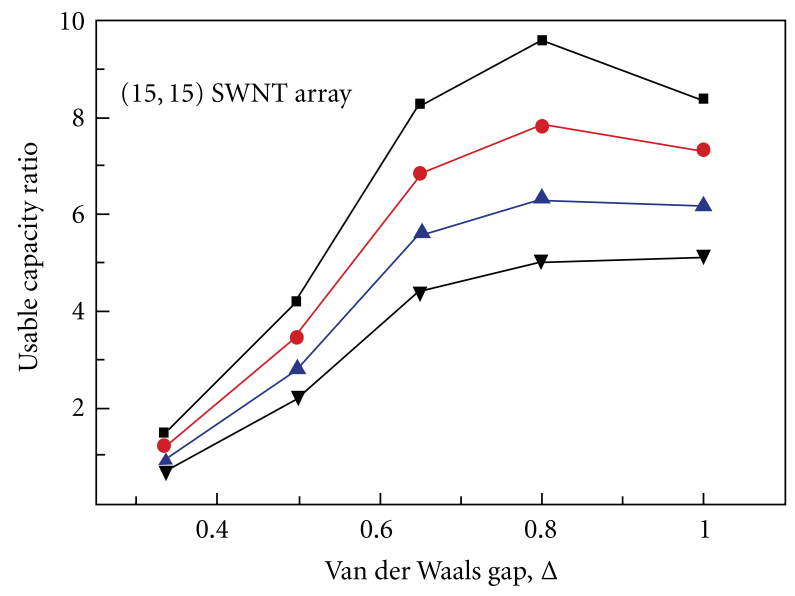

(a)

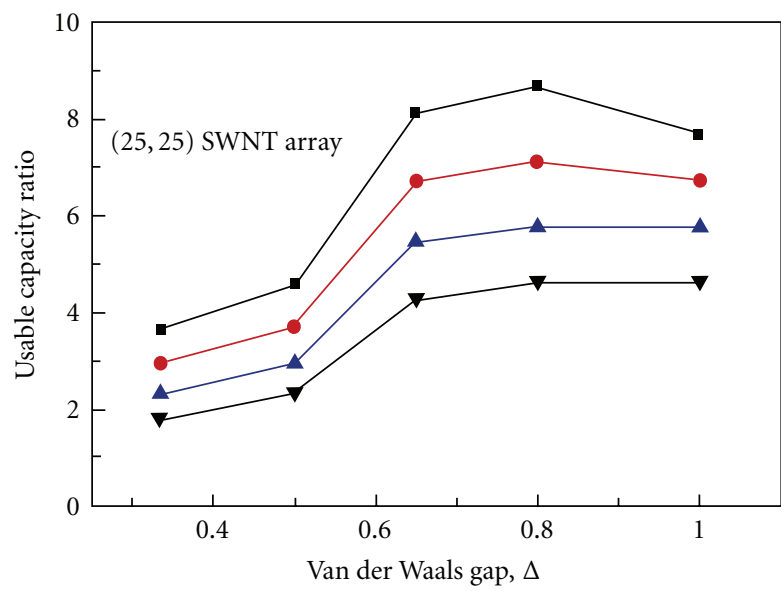

$-p=2.43 \mathrm{MPa} \rightarrow p=4.11 \mathrm{MPa}$
$\longrightarrow p=3.15 \mathrm{MPa} \rightarrow p=5.37 \mathrm{MPa}$

(c)

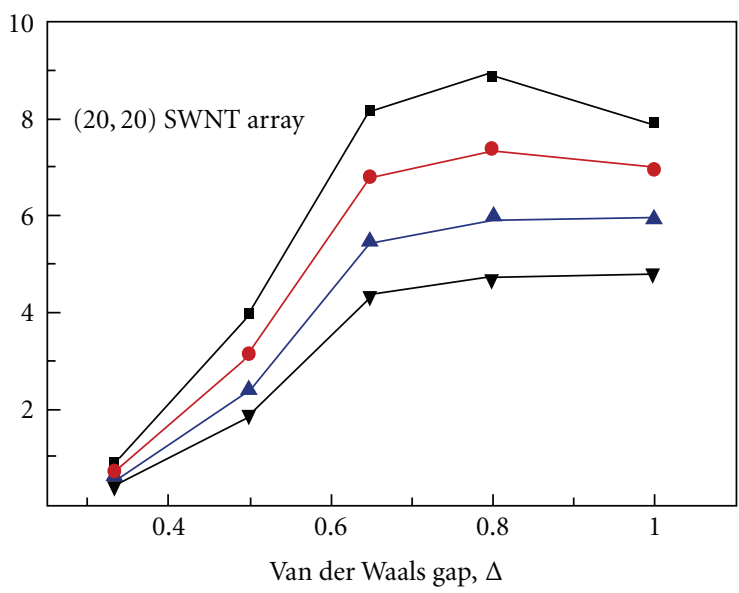

(b)

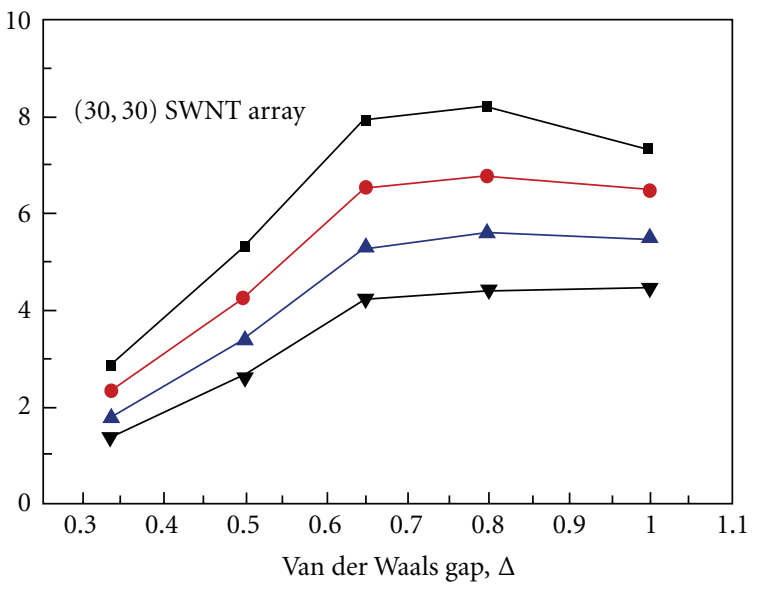

$-p=2.43 \mathrm{MPa} \rightarrow p=4.11 \mathrm{MPa}$
$-p=3.15 \mathrm{MPa} \rightarrow p=5.37 \mathrm{MPa}$

(d)

FIGURE 13: UCR for different SWCNT arrays with different VdW gaps at four pressure values. From [141]. 


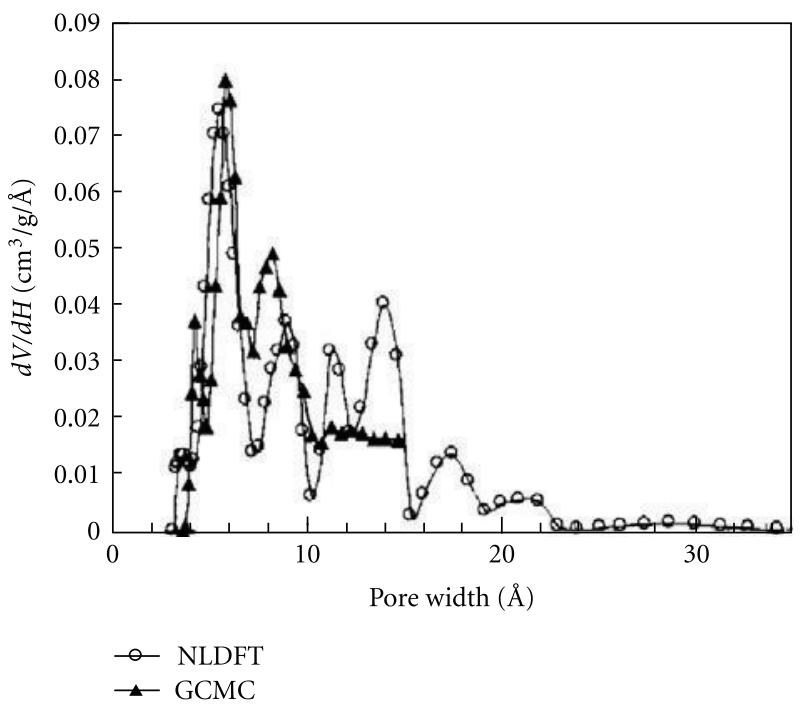

(a)

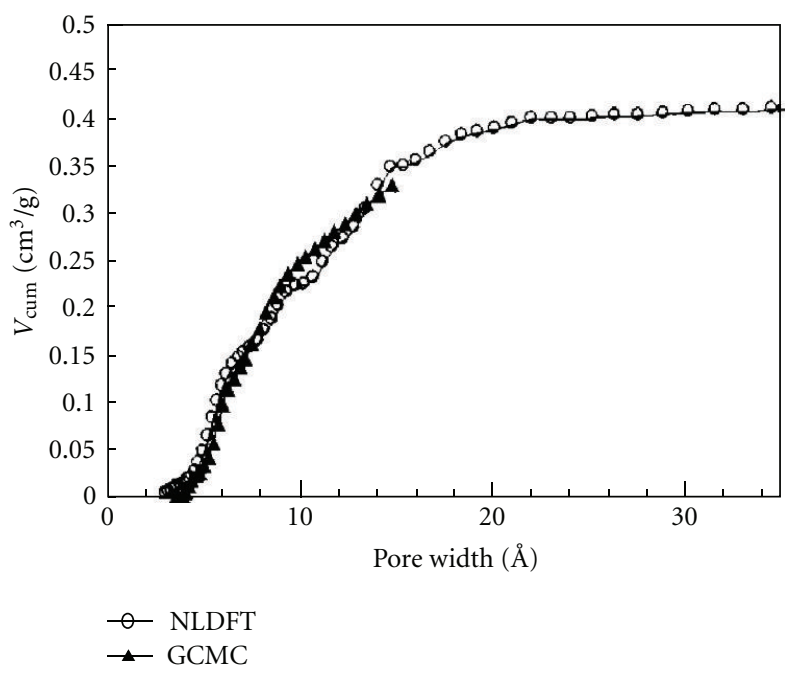

(c)

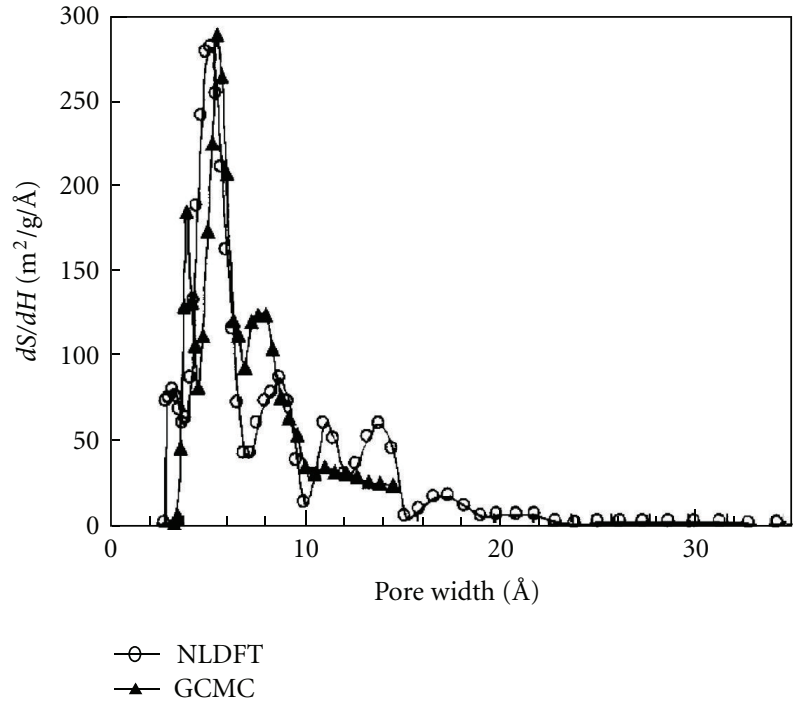

(b)

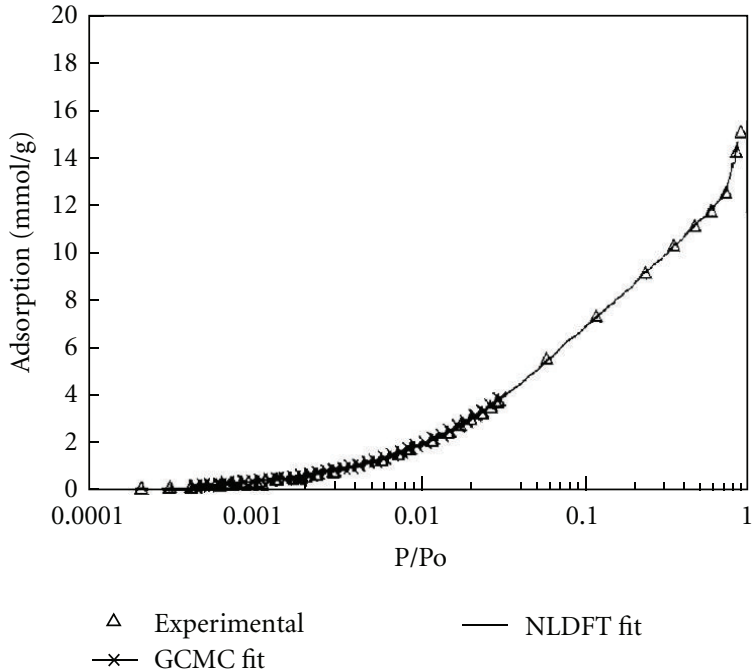

(d)

FIGURE 14: Differential pore volume (a), surface area distributions (b), cumulative pore volume distribution (c) of carbon B calculated from high-pressure $\mathrm{CO}_{2}$ adsorption isotherm at $273 \mathrm{~K}$ using NLDFT and three center GCMC models. Fit to the experimental isotherm (d) (experimental isotherm: points; theoretical fit: lines). From [154].

Moreover GCMC simulations using the Feynman-Hibbs quantum effective potential have evidenced important selectivity properties of CNT matrices. For instance, arrays of CNTs with diameter between 1.2-2.4 nm have large volumetric energy storage with respect to compression and evidence methane separation properties at RT and low pressure.

Methane storage in CMK-1 nanoporous structures has also been investigated by GCMC in combination with porosimetry. The isosteric heat values measured are within a broad range because of the heterogeneous nature of these materials [151].

5.2.2. Physical Adsorption of Other Gaseous Species. CNSs have been repeatedly proposed also for sensing exploiting gas chemisorption. However, sometimes the scenario is more complicated and, instead of chemisorption, strong physisorption is observed if accurate quantum chemistry methods are employed to describe the system. Moreover a significant part of the literature concerning physisorption of various gas species has been aimed to support porosimetry, especially concerning AC, MPC, and other disordered porous structures. Porosimetry has been often studied in connection with the storage problem to get reliable adsorption volume measurements. For instance, the adsorption isotherms for nitrogen, argon, carbon dioxide, and so forth, have been fitted by using GCMC or NLDFT using several interaction potentials, sometimes quantum corrected [152-155], in order to infer reliable pore size distributions from the experiments (see Figure 14) [154].

Nitrogen physical adsorption in CNT arrays has been studied at subcritical ( $77 \mathrm{~K}$ and $100 \mathrm{~K}$ ) and supercritical $(300 \mathrm{~K})$ temperatures showing that type II isotherms at 


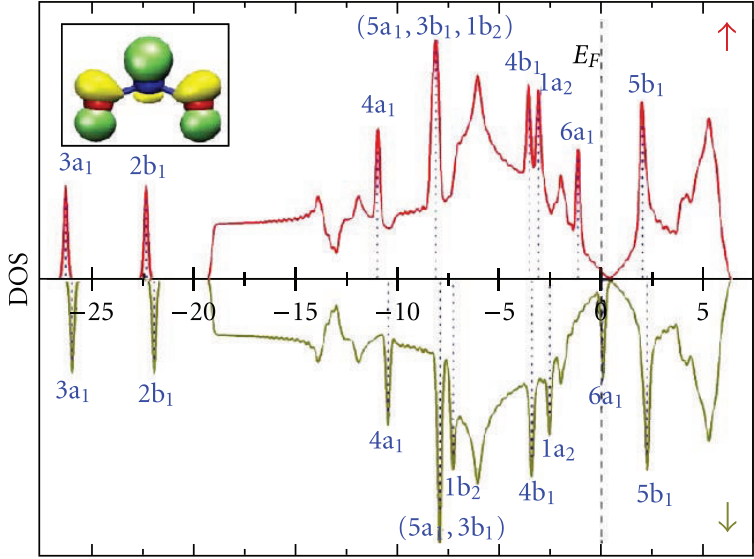

Energy (eV)

Figure 15: $\mathrm{NO}_{2}$ on graphene ( $\mathrm{N}$ atom is blue; $\mathrm{O}$ atoms are red). $\mathrm{HOMO} / \mathrm{LUMO}$ iso-density surfaces (a). Main panel: spin-polarized DOS. From [156]; copyright 2008 by the American Physical Society.

subcritical temperatures can be explained by taking into account the outer surface adsorption sites of the CNT bundles [57].

The rest of this subsection is dedicated to the physisorption of gaseous species, different from $\mathrm{H}_{2}$ and $\mathrm{CH}_{4}$, in graphene NSs and CNTs.

(1) Graphene. Thanks to its high conductivity, graphene is considered ideal for sensing purposes also because adsorbed species cause an enhanced response of this two-dimensional structure. Indeed charge carrier concentration can be varied by adsorption of various gases, even though the adsorbate identification may be problematic and accurate atomistic modelling is mandatory.

The graphene charge carrier concentration may be changed by charge transfer that depends on the HOMO and LUMO energy levels of the adsorbate with respect to the graphene Fermi energy. If the HOMO energy is above the graphene Fermi level, a negative charge is transferred from the molecule to the graphene whereas the opposite occurs if the energy of the LUMO is below the Fermi level. In addition, charge transfer is also partially determined by the mixing of the HOMO and LUMO with the graphene orbitals. In general charge transfer occur through bonding phenomena but sometimes a more complicated mixture of weak chemisorption and strong physisorption is evidenced.

$P$-type doping of graphene, for example, can be achieved by $\mathrm{NO}_{2}$ (or its dimer $\mathrm{N}_{2} \mathrm{O}_{4}$ ) adsorption [15], at large distances (between $0.34 \mathrm{~nm}$ and $0.39 \mathrm{~nm}$ ) with one distinction: the open shell monomer electron affinity is larger than the dimer one suggesting that paramagnetic molecules may act as strong dopants. This hypothesis has been checked by ab-initio calculations on several species, such as $\mathrm{H}_{2} \mathrm{O}$, $\mathrm{NH}_{3}, \mathrm{CO}, \mathrm{NO}_{2}$ and $\mathrm{NO}$ [156], evidencing that the charge transfer depends on the adsorbate orientation, it is nearly independent on the adsorption site and that paramagnetic molecules may not behave as strong dopants: indeed, while $\mathrm{NO}_{2}$ adsorption exhibits both chemisorption and

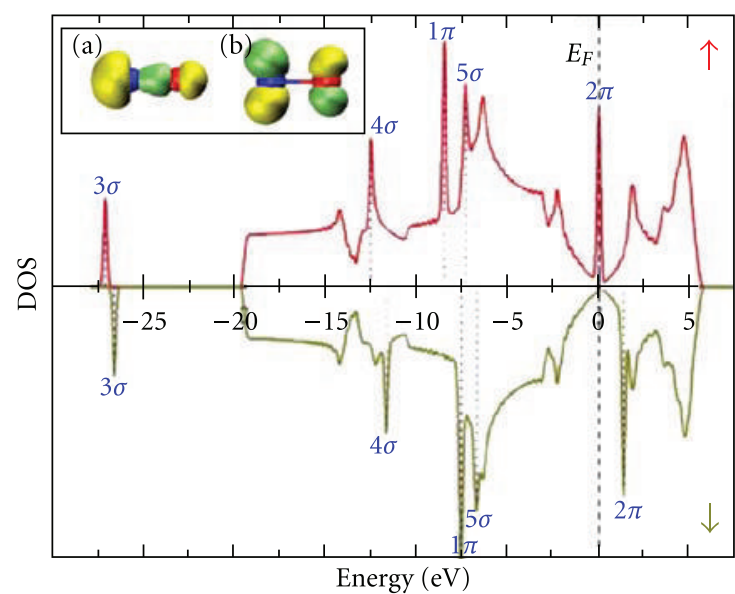

FIgure 16: $\mathrm{NO}$ on graphene ( $\mathrm{N}$ atom is blue; $\mathrm{O}$ atom is red). Inset: $5 \sigma$ (a) and HOMO/LUMO (b) iso-density surfaces. Main panel: spin-polarized DOS. From [156]; copyright 2008 by the American Physical Society.

physisorption characters with relatively strong doping $(-0.1 e)$ at large equilibrium distance $(0.361 \mathrm{~nm})$, no doping occurs for NO with negligible charge transfer $(<0.02 e$ at $0.376 \mathrm{~nm}$ distance). The different behavior of $\mathrm{NO}$ and $\mathrm{NO}_{2}$ on graphene can be understood looking at the spin-polarized DOS reported in Figures 15 and 16. The $\mathrm{NO}_{2}$ LUMO (6a1, $\uparrow$ ) is $0.3 \mathrm{eV}$ below the graphene Fermi energy and therefore charge is transferred from graphene to the molecule. In the NO case, on the contrary, the HOMO is only $0.1 \mathrm{eV}$ below the Fermi energy, is degenerate $\left(2 \pi_{x}, 2 \pi_{y}\right)$, and coincides with the LUMO. Thus the charge transfer is weak and the leading phenomenon is the mixing between the NO HOMO/LUMO and the graphene orbitals; as the hybridization above the Fermi energy prevails, the orbital mixing leads to charge transfer to graphene.

The stable configuration of triplet $\mathrm{O}_{2}$ on graphite has been modeled by accurate quantum chemistry techniques and high level DFT calculations $[157,158]$ evidencing that the choice of the exchange-correlation is crucial (LDA and $\mathrm{PBE}$ are inappropriate) and that spin unpolarized schemes are mandatory. A consensus was reached concerning the physisorption binding energy of $0.1 \mathrm{eV}$ at a distance of $0.31 \mathrm{~nm}$ and negligible charge transfer.

GNRs have been also functionalized with polar groups ( $\mathrm{COOH}, \mathrm{NH}_{2}, \mathrm{NO}_{2}, \mathrm{H}_{2} \mathrm{PO}_{3}$ ) evidencing enhanced adsorption for $\mathrm{CO}_{2}$ and $\mathrm{CH}_{4}$ physisorption $\mathrm{CO}_{2}$ binding is by far preferred over $\mathrm{CH}_{4}$ in hydrogen passivate GNRs [159].

A comparative study on diatomic halogen molecules on graphene has evidenced the crucial role played by the van der Waals interactions (that is more marked for species with large atomic radii) and the inadequacy of standard GGADFT [160].

(2) Carbon Nanotubes. The detection of physisorbed molecules on a SWCNT wall is an open problem of great technological interest and ab-initio calculations have been employed to this aim for several gaseous species such as 
$\mathrm{H}_{2} \mathrm{O}, \mathrm{NH}_{3}, \mathrm{CO}_{2}, \mathrm{CH}_{4}, \mathrm{NO}_{2}$, and $\mathrm{O}_{2}$ [48]. Most of the molecules studied are charge donors with small charge transfer $(0.010 e \sim 0.035 e$ per molecule $)$ and exhibit a weak binding energy $\left(E_{b} \leq 0.2 \mathrm{eV}\right)$ with no substantial electron density overlap between the adsorbate and the nanotube. On the contrary, acceptors such as $\mathrm{O}_{2}$ and $\mathrm{NO}_{2}$ exhibit a significant charge transfer, often accompanied by large adsorption energy, thus indicating that chemical and physical adsorption characters coexist.

Aromatic compounds interacting with CNSs show a similar uncertain nature of the bonding and their weak intermolecular forces, including van der Waals interactions, are often referred to as $\pi$-stacking interactions as they originate from the $\pi$-states of the interacting systems [50, 51]. Strictly speaking, $\pi$ molecular orbitals can be found only in planar systems such as graphene but for a CNT this concept still holds if one considers the $\pi$ bonds between the $p$-type orbitals, referred to as POAV ( $\pi$ Orthogonal Axis Vector), that are nearly orthogonal to the three $\sigma$ bonds between a carbon and its three neighbors. There are different metastable adsorption configurations of benzene on a CNT (see Figure 17), the most stable one in narrow CNTs being with the aromatic group above the middle of a $\mathrm{C}-\mathrm{C}$ bond (bridge position) that is different from the one on graphene (top position). Therefore the most favorable adsorption geometry should evolve from bridge to top as the nanotube diameter increases. In any case, the electronic structure calculation performed on these systems evidenced that the DOS is a superposition of the ones of the isolated benzene and the CNT, consistently with the fact that the $\pi$-stacking is accompanied by a very small binding energy. Consequently, the adsorption of benzene on a CNT is more appropriately classified as physisorption, although van der Waals interactions are not involved and a possible explanation is related to the misalignment of the POAV of neighboring carbon atoms. The adsorption of benzene derived molecules with different dipole moment and electron affinity properties, such as aniline $\left(\mathrm{C}_{6} \mathrm{H}_{5}-\mathrm{NH}_{2}\right)$, toluene $\left(\mathrm{C}_{6} \mathrm{H}_{5}-\mathrm{CH}_{3}\right)$, and nitrobenzene $\left(\mathrm{C}_{6} \mathrm{H}_{5}-\mathrm{NO}_{2}\right)$, on semiconducting $(8,0)$ SWCNT have been compared to the ones of benzene and of the "closedshell" functional groups $\mathrm{NH}_{3}, \mathrm{CH}_{4}$ and $\mathrm{CH}_{3} \mathrm{NO}_{2}$ [161]. The general trend found is that compounds with closed shells are always physisorbed with minor changes of the CNT electronic structure while both physisorption and chemisorption are possible for compounds with open shells. Moreover the adsorption is promoted by either the functional groups or the benzene rings depending on the configuration: for perpendicular configuration, the functional groups prevail while for the parallel configuration the interaction occurs through the $\pi$ electrons. Thus, in the first case the adsorption energies are at least $150 \mathrm{meV}$ smaller. The equilibrium distances are smaller than the $\mathrm{C}_{6} \mathrm{H}_{6}$ equilibrium distance and larger than the relevant functional groups ones, with the exception of toluene.

Similarly to the other CNSs, doping has been proposed to improve the physisorption of some molecular species on CNTs; B- and N-doped carbon nanotubes experience a large conductivity when exposed to $\mathrm{CO}$ or $\mathrm{H}_{2} \mathrm{O}$ [54]; more specifically, $\mathrm{CO}$ molecules are physisorbed onto $\mathrm{N}$-doped

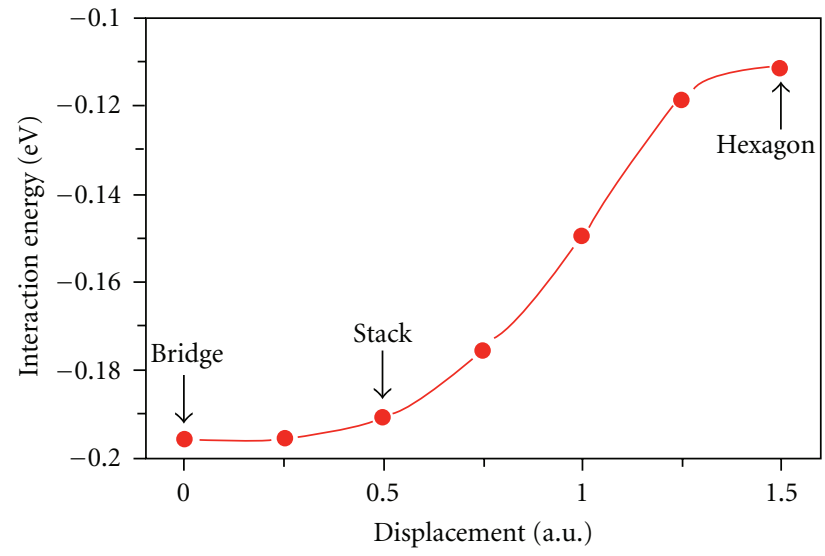

FIGURE 17: Energy curve of different benzene adsorption sites at the CNT side-wall (distance of $0.32 \mathrm{~nm}$ ). From [50]; copyright 2005 by the American Physical Society.

CNT because no charge transfer occurs while in the B-doped case chemisorption takes place (see below).

As for graphene, accurate quantum chemistry methods and high level DFT calculations have been performed to study $\mathrm{O}_{2}$ physisorption at the CNT "side-wall" $[157,158]$. Also in this case the calculation scheme may affect the results and the choice of the exchange-correlation functional is crucial. Using MP2 and other accurate quantum chemistry methods (DFT-B3LYP, DFT-PBE), it has been shown that $\mathrm{O}_{2}$ in a triplet state is physisorbed at a CNT, independently on the chiral vectors considered, at a distance of nearly $0.32 \mathrm{~nm}$ with no charge transfer and low binding energy (about $2.4 \mathrm{kcal} / \mathrm{mol}$ ).

\section{Gas Chemisorption in CNSs}

In this section we treat the systems where the adsorbatesubstrate interaction can be unambiguously ascribed to chemisorption with predominant bonding phenomena.

As in the previous section, we treat separately the case of the hydrogen chemical adsorption on some of the most recurrent carbon nanostructured adsorption media due to its potential importance in new technology and energy sources.

6.1. Hydrogen Chemisorption. Generally speaking, hydrogen chemisorption in carbon nanomaterials is not interesting for storage purposes because of the large binding energy involved that would make the experimental conditions for the adsorption/desorption cycles of little practical use. However, in storage experiments a significant amount of physisorbed hydrogen molecules could be involved in bonding phenomena when the hydrogen molecules get close to the carbons tanks to their thermal energy. Therefore hydrogen chemisorption must be considered explicitly.

6.1.1. Graphene. Chemisorption of atomic hydrogen on graphene leads to the appearance of a magnetic moment $[162,163]$ with a local lattice distortion nearby the adsorption site. The phenomenon gives rise to a strong Stoner ferromagnetism [164] with a magnetic moment of $1 \mu_{B}$ 
TABLE 1: Hydrogen adsorption on graphene: data selected from recent atomistic simulations.

\begin{tabular}{|c|c|c|c|c|c|c|c|}
\hline \multicolumn{2}{|c|}{ Adsorption } & \multicolumn{2}{|c|}{ Doping/site } & \multirow{2}{*}{$\begin{array}{l}E_{b}(\mathrm{eV}) \\
-0.086\end{array}$} & \multirow{2}{*}{$\frac{d_{0}(\mathrm{~nm})}{0.268}$} & \multirow{2}{*}{$\frac{\text { DFT model }}{\text { LDA }}$} & \multirow{2}{*}{$\frac{\text { Reference }}{[11]}$} \\
\hline $\mathrm{H}_{2}$ & $\mathrm{P}$ & & $\mathrm{H}$ & & & & \\
\hline $\mathrm{H}$ & $\mathrm{C}$ & & $\mathrm{T}$ & -0.200 & & LDA & {$[12]$} \\
\hline $\mathrm{H}$ & $\mathrm{C}$ & & $\mathrm{T}$ & -0.670 & 0.113 & GGA & {$[166]$} \\
\hline \multirow{2}{*}{$\mathrm{H}_{2}$} & \multirow{2}{*}{$\mathrm{P}$} & $\mathrm{Li}$ & $\mathrm{H}$ & -0.988 & 0.213 & \multirow{2}{*}{ B3YLP } & \multirow{2}{*}[134]{} \\
\hline & & $\mathrm{K}$ & $\mathrm{H}$ & -0.701 & 0.304 & & \\
\hline \multirow{2}{*}{$\mathrm{H}_{2}$} & \multirow{2}{*}{$\mathrm{P}$} & & $\mathrm{H}$ & -0.092 & 0.270 & \multirow{2}{*}{ LDA } & \multirow{2}{*}{ [13] } \\
\hline & & $\mathrm{Li}$ & $\mathrm{H}$ & -0.184 & 0.259 & & \\
\hline $\mathrm{H}$ & $\mathrm{C}$ & & $\mathrm{T}$ & -1.441 & 0.110 & GGA & {$[162]$} \\
\hline \multirow{2}{*}{$\mathrm{H}_{2}$} & \multirow{2}{*}{$\mathrm{P}$} & $\mathrm{Ca}$ & Edge & -0.2 & \multirow{2}{*}{ - } & \multirow{2}{*}{ GGA } & \multirow{2}{*}[135]{} \\
\hline & & $\mathrm{Ca} / \mathrm{B}$ & Edge & -0.2 & & & \\
\hline $\mathrm{H}_{2}$ & $\mathrm{P}$ & $\mathrm{Li} / \mathrm{B}$ & $\mathrm{H}$ & -0.2 & - & GGA & {$[136]$} \\
\hline
\end{tabular}

In the "Adsorption" column, $\mathrm{C}$ and $\mathrm{P}$ stand for chemisorption and physisorption, respectively (a chemisorbed hydrogen molecule involves its dissociation). In the "Doping/site" column, H, T, and B indicate adsorption on a Hollow, Top, and Bridge site, respectively. Blank spaces in the column of binding energies and/or equilibrium distances indicate the missing of the corresponding values in the original paper. Further details are referred in the cited articles.
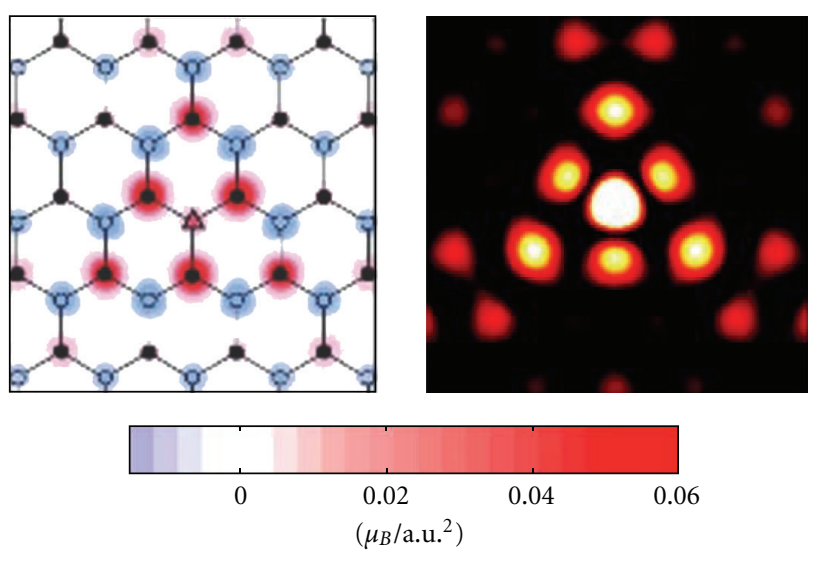

(a)

(b)

FIGURE 18: Spin-density projection on the graphene plane around the hydrogen chemisorption site (triangle) (a); carbon atoms in the $\alpha$ sublattice $(\circ)$ and the $\beta$ sublattice $(\bullet)$ are distinguished. Simulated STM image (b). Adapted from [163]; copyright 2007 by the American Physical Society.

per hydrogen atom, as evidenced by the spin-density in Figure 18. According to the Stoner picture, magnetic ordering is driven by the exchange energy between the $p_{z}$-orbitals of ther adsorption sites and either ferromagnetism or antiferromagnetism occurs if the $\mathrm{H}$ derived bound states are located at equivalent or different lattice sites. The energy difference between different adsorption sites, namely top, bridge and hollow, is small and hydrogen diffusion occurs even at low temperature; as a consequence, two $\mathrm{H}$ atoms may easily recombine and form molecular hydrogen that is immediately desorbed from the graphene $[165,166]$.

On the other hand, full hydrogen coverage of both sides of an isolated graphene layer form a stable structure where each carbon undergoes a hybridization transition from $\mathrm{sp}^{2}$ to $\mathrm{sp}^{3}$. The situation is different at intermediate coverage and strongly depends on the overall magnetization as indicated by the linear dependence of the secondary $\mathrm{H}$ adsorption binding energy on the "site-integrated" magnetization [167]. Therefore, at least at low temperature, it would be possible to control the adsorption dynamics of $\mathrm{H}$ atoms by tuning the substrate magnetization. In Table 1 we report some selected data concerning the properties of hydrogen adsorption on graphene.

6.1.2. Fullerenes. Novel fullerene organo-metallic molecules have been deeply studied for hydrogen storage. To this aim, light elements, either in interstitial ( $\mathrm{Li}$ and $\mathrm{F}$ ) or in substitutional sites $(\mathrm{N}, \mathrm{B}$ and $\mathrm{Be})$, have been investigated as doping species of $\mathrm{C}_{36}$ and $\mathrm{C}_{60}$ by means of LDA and GGA $a b$ initio total energy calculations [23]. Fullerenes doped with B and $\mathrm{Be}$ at substitutional sites exhibits large hydrogen binding energies ( 0.40 and $0.65 \mathrm{eV}$, respectively) due to the strong interaction between the $\mathrm{B}(\mathrm{Be}) p_{z}$-orbital and the hydrogen $\sigma$ molecular orbital (MO).

The orbital interaction, evidenced in Figure 19, causes the splitting of the $\mathrm{H}_{2} \sigma$ MO bonding state below the Fermi level, whereas the $\mathrm{B} p_{z}$-state, that is normally located in the range $1-3 \mathrm{eV}$ above the Fermi energy for B-doped fullerenes, shifts to higher energy values. Similar phenomena occur also for $\mathrm{C}_{35} \mathrm{Be}-\mathrm{H}_{2}$.

The charge transfer analysis, performed along the direction orthogonal to the hydrogen axis (see Figure 20), shows that, in the case of B, only few electrons are involved in the formation of a "three-center" bond, in contrast with the Be case; therefore the hydrogen adsorption energy $E_{a}$ for Be is larger and nearly insensitive to the number of adsorbed molecules than the one for B (see Figure 21) confirming that highly localized $p_{z}$-orbitals are needed for not dissociative adsorption. In the B case, moreover, hydrogen desorption may occur more easily. In spite of the advantages of Be over B, however, a controlled Be doping is difficult to obtain also because of its toxicity while B-doped fullerenes has been already synthesized. In particular, first principles molecular dynamics simulations have revealed that $\mathrm{C}_{54} \mathrm{~B}_{6}$ hydrogenation is unstable and that the reaction path (see Figure 22) causes the desorption to occur within the 


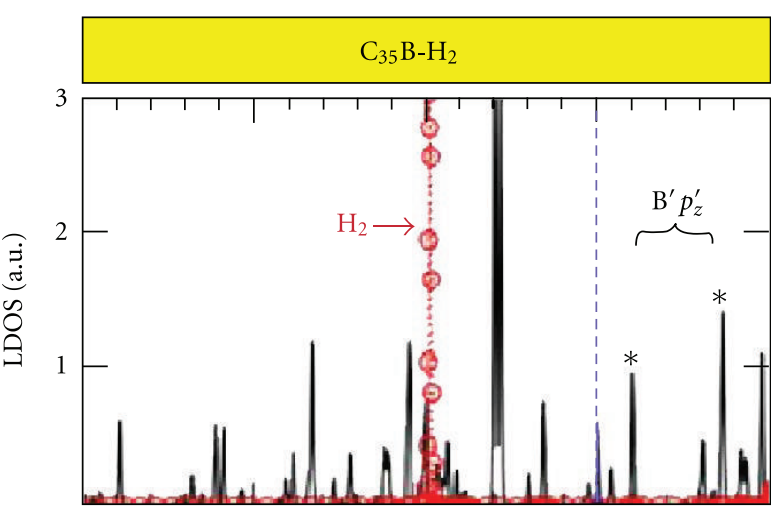

(a) Nonbonding

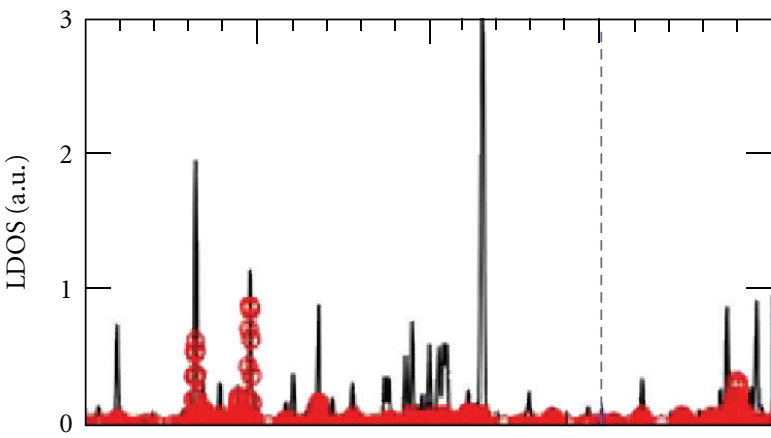

(c) Bonding

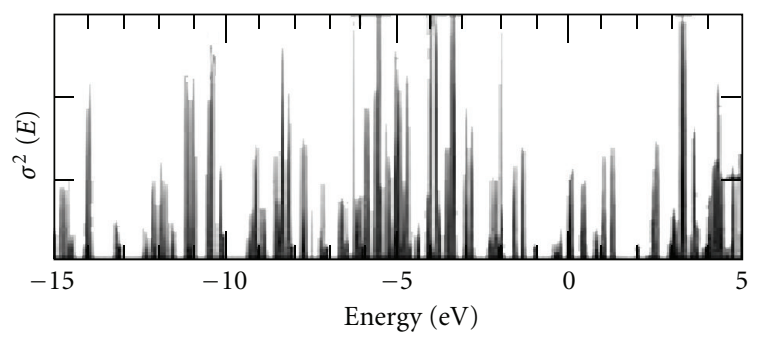

(e) Carbon

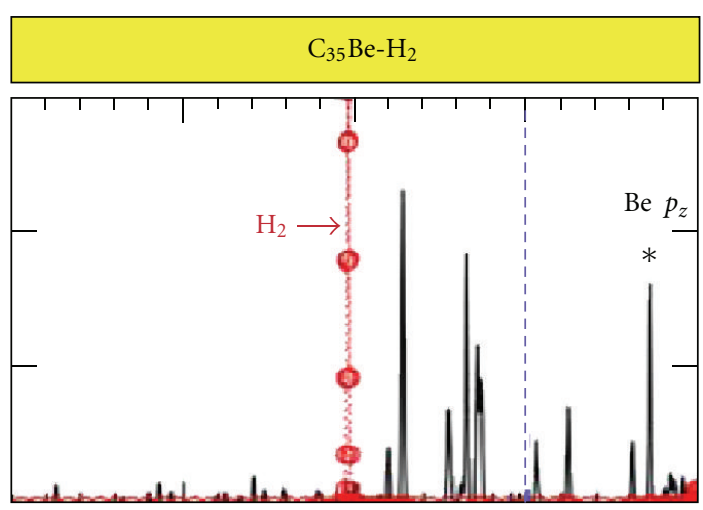

(b) Nonbonding

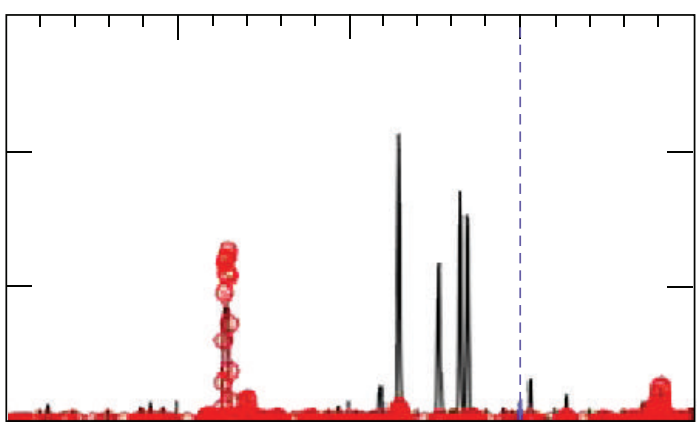

(d) Bonding

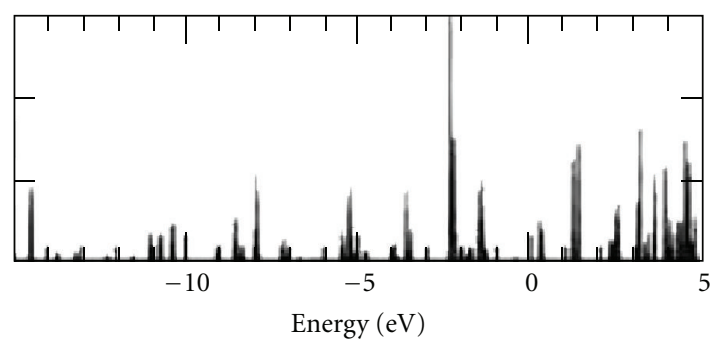

(f) Carbon

FIgure 19: The calculated local density of states (LDOS) for $\mathrm{C}_{35} \mathrm{~B}(\mathrm{Be})-\mathrm{H}_{2}(\mathrm{a})-(\mathrm{d})$. The $\mathrm{B}(\mathrm{Be})$ and the $\mathrm{H}_{2}$ LDOS are solid lines and open circles, respectively. Squared differences between the nonbonding and bonding states due to $\mathrm{H}_{2}$ sorption (e) and (f). From [23]; copyright 2006 by the American Physical Society.

picosecond timescale [168]. Among the other doping species investigated, $\mathrm{Si}$ is interesting because industrial $\mathrm{C}_{60}$ synthesis is performed on silicon surface [31]. $\mathrm{H}_{2}$ adsorption on the Si site occurs at a distance of $d_{0}=0.256 \mathrm{~nm}$ with $0.15 \mathrm{eV}$ binding energy that indicates an intermediate state between physical and chemical adsorption. A similar situation is found also in $\mathrm{Ni}$-doped fullerenes [27] where the $\mathrm{Ni}$ valence states are depleted by about half an electron resulting in large van der Waals interactions with a gravimetric ratio of $6.8 \mathrm{wt} \%$. From Table 2, where are reported some selected results concerning hydrogen adsorption on fullerenes, it emerges that atomistic simulations predict $\mathrm{Si}, \mathrm{Li}, \mathrm{Ca}$, and $\mathrm{Sr}$ as the doping species that could enhance most of the hydrogen uptake in "fullerene-like" CNSs.

6.1.3. Carbon Nanotubes. Some of the most notable results found in the literature concerning hydrogen adsorption on a
CNT are collected in Table 3 including data on both chemical and physical adsorption. Following experimental evidences, hydrogen chemisorption has been treated by DFT total energy calculations studying two energetically favored sites where atomic hydrogen is chemisorbed [39]. Both of them are accompanied by an $\mathrm{sp}^{2}$ to $\mathrm{sp}^{3}$ hybridization transition, the most stable being characterized by the hydrogen atoms alternating outside and inside on the tube "side-wall" (zigzag type). Hydrogen half-full coverage of CNTs has been investigated with high accurate quantum chemistry models showing that this configuration is more stable with respect to the full coverage case and suggesting that the deformations induced by the adsorption of $\mathrm{H}$ atoms can affect the stability of CNTs [38].

However, many experimental studies have shown that the chemisorbed hydrogen storage capacity on pure CNT media is less than $0.01 \mathrm{wt} \%$ at room temperature, resulting 


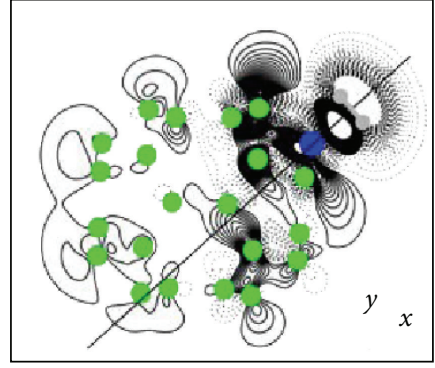

(a)

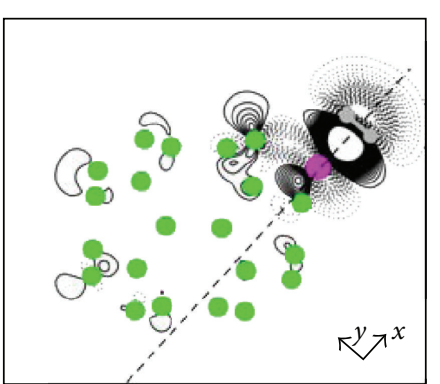

(b)

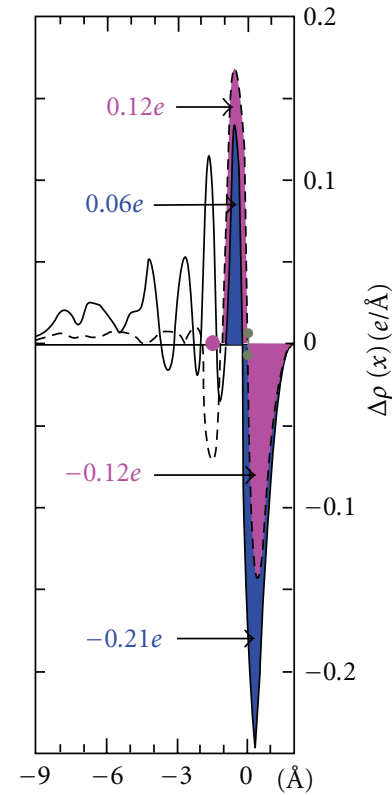

(c)

FIGURE 20: Differential planar electron density for B (a) and Be (b), respectively: solid and dotted contours indicate electron accumulation and depletion, respectively. (c) Differential planar electron density along the $x$-axis for $\mathrm{B}$ and $\mathrm{Be}$. The positions of the $\mathrm{H}_{2}$ and $\mathrm{B}(\mathrm{Be})$ are indicated. From [23]; copyright 2006 by the American Physical Society.

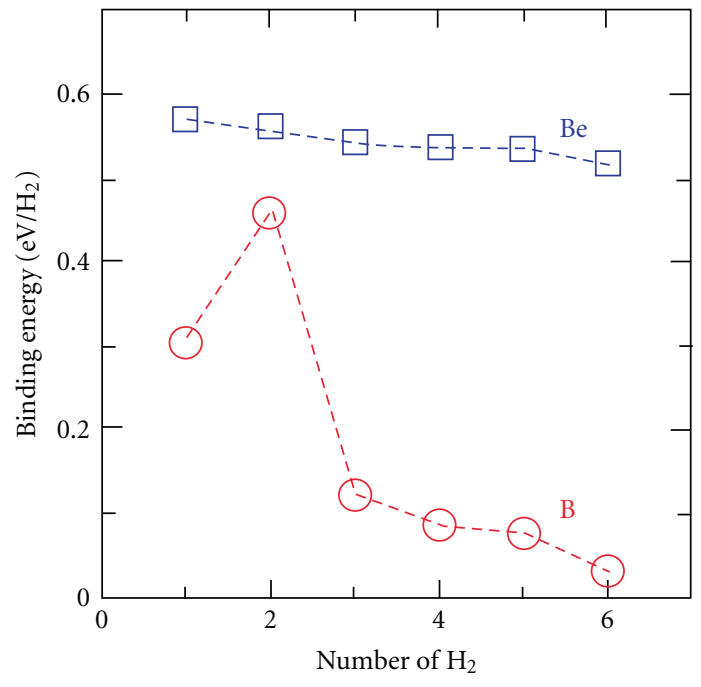

(a)

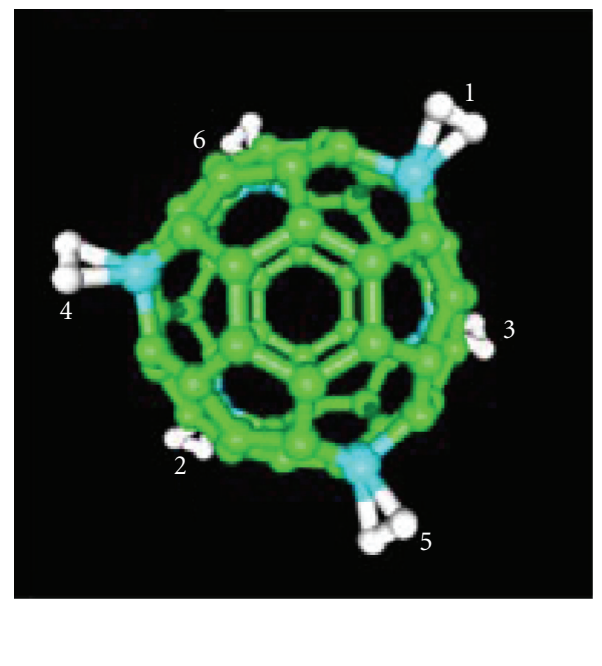

(b) $\mathrm{C}_{54} \mathrm{~B}_{6}-6 \mathrm{H}_{2}$

Figure 21: Binding energy for each added $\mathrm{H}_{2}$ as a function of number of adsorbed $\mathrm{H}_{2}$ molecules (a) and $\mathrm{C}_{54} \mathrm{~B}_{6}$ with $6 \mathrm{H}_{2}$ molecules (b). From [23]; copyright 2006 by the American Physical Society.

impractical for storage applications. As for fullerenes, CNT doping with metallic impurities can improve the situation, as evidenced using $\mathrm{Ti}$ [28]. Unpolarized spin density calculations have shown that, while an $\mathrm{H}_{2}$ molecule approaches a Ti coated zigzag CNT, the energy decreases in two steps, the first one due to a charge overlap resulting in an increased attraction between $\mathrm{H}_{2}$ and $\mathrm{Ti}$ and the second one related to the $\mathrm{H}_{2}$ molecule dissociation with a final binding energy of $0.83 \mathrm{eV}$. This scenario is quite different from the case of $\mathrm{Ti}$ decorated fullerenes (where $\mathrm{H}_{2}$ is simply physisorbed) because of the different coordination numbers of $\mathrm{Ti}$ in the two cases: in the CNT case, indeed, the larger Ti charge is responsible for the $\mathrm{H}_{2}$ dissociation and the subsequent chemisorption. The first $\mathrm{H}_{2}$ chemisorption event is followed by the physisorption of three other hydrogen molecules on the same Ti site. Alternatively four hydrogen molecules can 


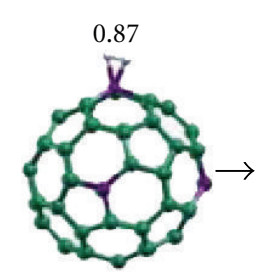

(a)

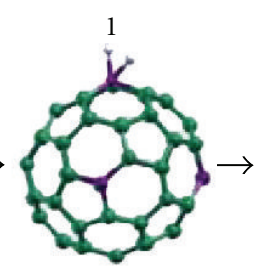

(b)

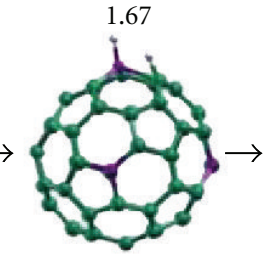

(c)

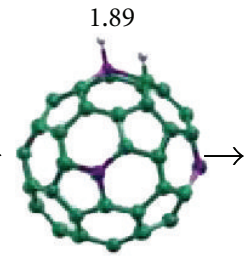

(d)

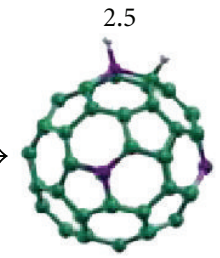

(e)

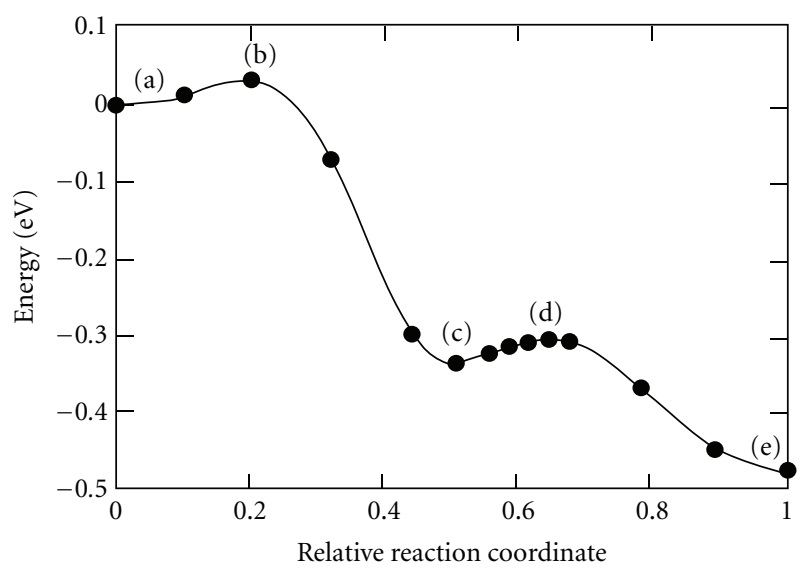

(f)

Figure 22: Minimum-energy path of dissociation of a $\mathrm{H}_{2}$ molecule obtained from the CI-NEB calculation (a)-(e). Numbers are the distance between the hydrogen atoms in angstroms. (f) The calculated minimum energy path of the dissociation of the $\mathrm{H}_{2}$ molecule. Two activation energy barriers at 32 and $28 \mathrm{meV}$ are found, respectively, from left to right. From [168] copyright 2008 by the American Physical Society.

be simply physisorbed at a Ti decorated site in a low energy $(0.1 \mathrm{eV}$ lower than the previous one) and high symmetry configuration. In this case, the bonding mechanism is quite similar to the Dewar, Chatt and Duncanson model because it implies the donation of $1 e$ to the $4 \mathrm{H}_{2} \sigma^{*}$ antibonding molecular orbital (hybridized with the Ti $d$-state) followed by the transfer of $0.4 e$ to an empty Ti $d$-orbital. The above scenario is schematically drawn in Figure 23.

Because Pt surfaces can adsorb gaseous molecules reversibly, DFT calculations of molecular hydrogen on Ptdoped armchair CNTs have been performed $[44,111]$ showing that chemisorption is accompanied by an oxidative addition to Pt involving its $5 d$-orbital. However Pt clustering may occur that favors molecular hydrogen dissociation and reversible atomic hydrogen chemisorption [111].

Pd decoration of SWCNT behaves similarly [45] with a storage capacity of about $3 \mathrm{wt} \%$. The most stable configuration exhibits both the physical and chemical adsorption characters with five hydrogen molecules adsorbed onto two adjacent $\mathrm{Pd}$ atoms through a partial hybridization between the $\mathrm{H}_{2} s$-orbitals and the $\mathrm{Pd} d$-orbitals.

Recent ab-initio molecular dynamics simulations of nitrogen decorated SWCNTs [169] have evidenced that hydrogen chemisorption occurs at $77 \mathrm{~K}$ and is stable at $300 \mathrm{~K}$ while physisorption is enhanced at both the temperatures. These results, obtained within a DFT-LDA scheme, have also evidenced that $0 \mathrm{~K}$ ground state properties of such systems should be revised at higher temperature where desorption or enhanced chemisorption may occur affecting storage. The scenario emerging from the above discussion and the results resumed in Table 3 is that TMs may enhance physisorption at the expense of having chemisorption on the CNT walls.

6.2. Gas Chemisorption for Sensing. As mentioned in the Introduction, gas chemical adsorption in CNSs has been studied focusing on gas sensing. Of course, the computational techniques required are quantum chemistry techniques, DFT calculations, and similar. Due to the amount of the literature found, we have just treated nanostructured graphene and CNTs.

6.2.1. Graphene-Based NSs. Graphene charge carrier concentration can be strongly modified by gas chemisorption. Therefore, the electronic and magnetic properties of GNRs can be modified by edge functionalization or substitutional doping. However GNRs with well controlled saturated edges without dangling bonds (DBs) are far to be produced; these defects usually enhance the covalent bonding of chemical groups and molecules thus playing a critical role in the feasibility of using such carbon-based nanostructures as gas sensors. Semiconducting armchair GNRs (AGNRs) are preferred with respect to zig-zag GNRs (ZGNRs) since gas molecule adsorption is expected to induce little modifications on the electronic properties of metallic ZGNRs. 
TABLE 2: Hydrogen adsorption on fullerenes: data selected from recent atomistic simulations.

\begin{tabular}{|c|c|c|c|c|c|c|c|}
\hline \multicolumn{2}{|c|}{ Adsorption } & \multicolumn{2}{|c|}{ Molecule/dopant } & \multirow{2}{*}{$\begin{array}{c}E_{b}(\mathrm{eV}) \\
-0.18\end{array}$} & \multirow{2}{*}{$\begin{array}{c}d_{0}(\mathrm{~nm}) \\
0.207\end{array}$} & \multirow{2}{*}{$\begin{array}{c}\text { DFT model } \\
\text { GGA }\end{array}$} & \multirow{2}{*}{$\begin{array}{c}\text { Reference } \\
{[25]} \\
\end{array}$} \\
\hline $\mathrm{H}_{2}$ & $\mathrm{P}$ & $\mathrm{C}_{60}$ & $\mathrm{Li}$ & & & & \\
\hline \multirow{6}{*}{$\mathrm{H}_{2}$} & $\mathrm{P}$ & \multirow{6}{*}{$\mathrm{C}_{36}$} & & -0.07 & & \multirow{6}{*}{ LDA } & \\
\hline & $\mathrm{P}$ & & $\mathrm{Li}$ & -0.07 & & & \\
\hline & $\mathrm{P}$ & & $\mathrm{F}$ & -0.04 & & & \\
\hline & $\mathrm{P}$ & & $\mathrm{N}$ & -0.06 & & & {$[23]$} \\
\hline & $\mathrm{C}$ & & B & -0.39 & & & \\
\hline & $\mathrm{C}$ & & $\mathrm{Be}$ & -0.65 & & & \\
\hline \multirow{3}{*}{$\mathrm{H}_{2}$} & \multirow{3}{*}{$\mathrm{P}$} & \multirow{3}{*}{$\mathrm{C}_{60}$} & $\mathrm{Li}$ & -0.115 & 0.220 & \multirow{3}{*}{ B3LYP } & \multirow{3}{*}[24]{} \\
\hline & & & $\mathrm{Na}$ & -0.074 & 0.258 & & \\
\hline & & & $\mathrm{K}$ & -0.0520 & 0.308 & & \\
\hline $\mathrm{H}_{2}$ & $\mathrm{C}$ & $\mathrm{C}_{60}$ & $\mathrm{~B}$ & -0.46 & 0.137 & LDA & {$[168]$} \\
\hline \multirow{2}{*}{$\mathrm{H}_{2}$} & \multirow{2}{*}{$\mathrm{P}$} & \multirow{2}{*}{$\mathrm{C}_{60}$} & $\mathrm{Ca}$ & $-0.222 /-0.413$ & & GGA/LDA & \multirow{2}{*}[26]{} \\
\hline & & & $\mathrm{Sr}$ & -0.200 & & GGA & \\
\hline $\mathrm{H}_{2}$ & $\mathrm{C} / \mathrm{P}$ & $\mathrm{C}_{60}$ & $\mathrm{Si}$ & -0.150 & 0.256 & B3LYP & [31] \\
\hline
\end{tabular}

In the "Adsorption" column C and P stand for chemisorption and physisorption, respectively (a chemisorbed hydrogen molecule involves its dissociation). Blank spaces in the column of binding energies and/or equilibrium distances indicate the missing of the corresponding values in the original paper. Further details are referred in the cited articles.

TABLE 3: Hydrogen adsorption on a CNT: data selected from recent atomistic simulations.

\begin{tabular}{|c|c|c|c|c|c|c|c|c|}
\hline \multicolumn{2}{|c|}{ Adsorption } & \multicolumn{2}{|c|}{ SWCNT/dopant } & \multirow[t]{2}{*}{ Site } & \multirow{2}{*}{$\begin{array}{c}E_{b}(\mathrm{eV}) \\
-0.043\end{array}$} & \multirow{2}{*}{$\begin{array}{c}d_{0}(\mathrm{~nm}) \\
0.29\end{array}$} & \multirow{2}{*}{$\begin{array}{c}\text { DFT model } \\
\text { LDA }\end{array}$} & \multirow{2}{*}{$\begin{array}{c}\text { Reference } \\
{[38]}\end{array}$} \\
\hline $\mathrm{H}_{2}$ & $\mathrm{P}$ & $(5,5)$ & - & & & & & \\
\hline $\mathrm{H}$ & $\mathrm{C}$ & $(5,5)$ & - & Full coverage & $-2.10 /-1.75$ & 0.112 & LDA/GGA & {$[40]$} \\
\hline $\mathrm{H}$ & $\mathrm{C}$ & $(5,5)$ & - & Caps coverage & -2.94 & - & AM1 & {$[39]$} \\
\hline \multirow{3}{*}{$\mathrm{H}_{2}$} & & $(10,0)$ & & & -0.113 & 0.281 & \multirow{3}{*}{ LDA } & \multirow{3}{*}[48]{} \\
\hline & $\mathrm{P}$ & $(5,5)$ & - & & -0.084 & 0.319 & & \\
\hline & & $(17,0)$ & & & -0.049 & 0.255 & & \\
\hline \multirow{3}{*}{$\mathrm{H}_{2}$} & $\mathrm{P}$ & & - & & -0.057 & 0.310 & \multirow{3}{*}{ GGA + VdW } & \multirow{3}{*}[111]{} \\
\hline & $\mathrm{P}$ & $(8,0)$ & $\mathrm{Li}$ & & - & 0.210 & & \\
\hline & $\mathrm{C}$ & & $\mathrm{Pt}$ & & -1.18 & 0.157 & & \\
\hline $\mathrm{H}_{2}$ & $\mathrm{C}$ & $(8,0)$ & $\mathrm{Ti}$ & & -0.830 & 0.173 & GGA & {$[28]$} \\
\hline $\mathrm{H}_{2}$ & $\mathrm{C}$ & $(5,5)$ & $\mathrm{Pt}$ & & -0.491 & 0.166 & GGA & {$[44]$} \\
\hline \multirow{2}{*}{$\mathrm{H}_{2}$} & \multirow{2}{*}{$\mathrm{P}$} & \multirow{2}{*}{$(10,10)$} & $\mathrm{Li}$ & & -0.180 & 0.210 & \multirow{2}{*}{ GGA } & \multirow{2}{*}[43]{} \\
\hline & & & Li-peapod & & -0.217 & 0.208 & & \\
\hline $\mathrm{H}_{2}$ & $\mathrm{C} / \mathrm{P}$ & $(8,0)$ & $\mathrm{Pd}$ & & -0.800 & - & GGA & {$[45]$} \\
\hline
\end{tabular}

In the "Adsorption" column C and P stand for chemisorption and physisorption, respectively (a chemisorbed hydrogen molecule involves its dissociation). Blank spaces in the column of binding energies and/or equilibrium distances indicate the missing of the corresponding values in the original paper. Further details are referred in the cited articles.

Adsorption of many gas molecules $\left(\mathrm{CO}, \mathrm{NO}, \mathrm{NO}_{2}, \mathrm{O}_{2}\right.$, $\mathrm{CO}_{2}$, and $\mathrm{NH}_{3}$ ) has been studied by spin-polarized GGADFT total energy calculations [170]: among the different gaseous species considered, only $\mathrm{NH}_{3}$ has been found to greatly enhance the AGNRs conductance after chemical adsorption; in this case a semiconducting/metallic transition occurs thus suggesting that, in principle, a "GNR-based" junction can be used to detect $\mathrm{NH}_{3}$ (see Figure 24) by I$V$ measurements. Indeed, the GNR sensor exhibits a semiconducting behavior when no gas molecule is adsorbed while, after $\mathrm{NH}_{3}$ adsorption, the current increases linearly with the applied bias evidencing a metallic behavior.

Molecular adsorption at vacancy sites in nanostructured graphene has been also investigated as a possible sensing mechanism and this system is expected to behave similarly to GNRs. Vacancies and divacancies can be introduced by ion or electron irradiation under vacuum conditions and their passivation is of crucial interest in the development of graphene nanoelectronics. Divacancies in graphene have been passivated using several possible gaseous species, such 


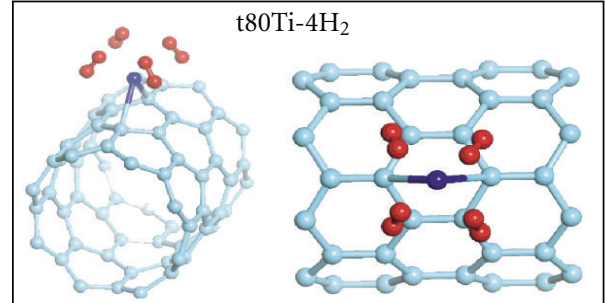

(a)

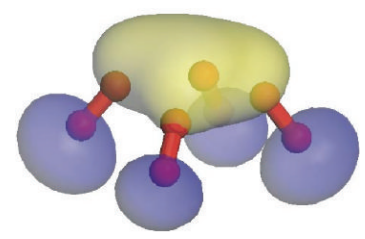

(c)

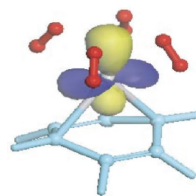

(d)

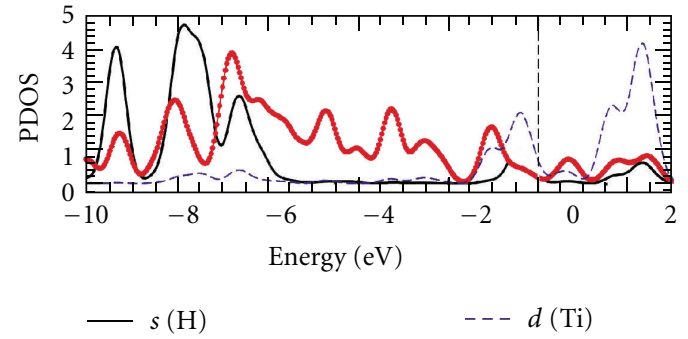

$\longrightarrow s(\mathrm{H})$

(b)

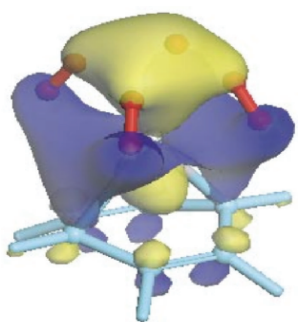

(e)

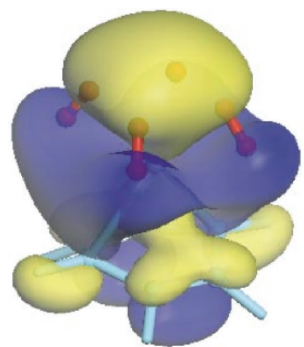

(f)

Figure 23: (a) Two different views of optimized structures of $t 80 \mathrm{Ti}-4 \mathrm{H}_{2}$; (b) PDOS at the $\Gamma$ point; (c) $\sigma^{*}$ antibonding orbital of four $\mathrm{H}_{2}$ complexes; (d)-(f) isosurface of the state just below $E_{\mathrm{F}}$ at three different values: at $\Psi=0.08 \mathrm{Ti} d$-orbital is visible; at $\Psi=0.04 \mathrm{Ti}-d$-orbital, two carbon $\pi$-orbitals, and $4 \mathrm{H}_{2} \sigma^{*}$ antibonding are hybridized; At $\Psi=0.02$ it emerges that the other four carbon atoms are also involved in the bonding. From [28]; copyright 2005 by the American Physical Society.

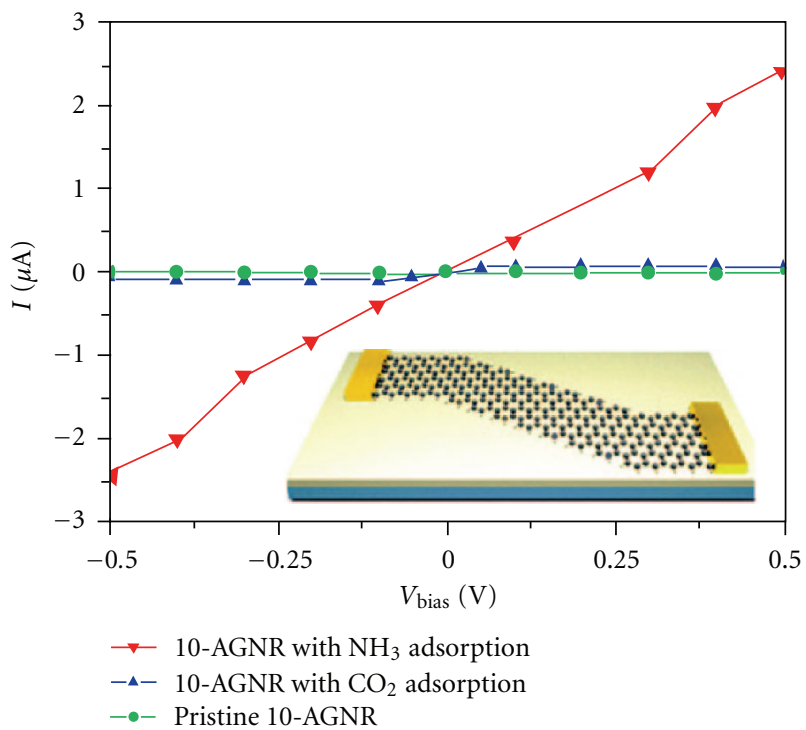

FIGURE 24: $I-V_{\text {bias }}$ curves for the GNR sensor before and after the adsorption of $\mathrm{NH}_{3}$ and $\mathrm{CO}_{2}$. The inset shows schematics of such a GNR sensor, consisting of one 10-AGNR (detection region) and two metallic 7-ZGNRs leads. From [170].

as $\mathrm{O}_{2}, \mathrm{~N}_{2}, \mathrm{~B}_{2}, \mathrm{CO}$, and $\mathrm{H}_{2} \mathrm{O}$, in the context of DFT ab-initio calculations [14]. In the particular case of $\mathrm{N}_{2}$, for instance, the molecule undergoes dissociation and subsequent chemical adsorption on the graphene layer resulting in substitutional $\mathrm{N}$ impurities that introduce extra carriers and change the charge transport properties. A summary of the most important results discussed here can be found in Table 4 where we have included also data from physisorption studies. It is quite evident that chemical adsorption at divacancies is significantly stronger than adsorption at dangling bonds.

6.2.2. Carbon Nanotubes. As steam reforming of natural gas is employed to produce hydrogen, the interest in $\mathrm{CH}_{4}$ and hydrocarbons adsorption has grown rapidly. However the chemical functionalization of a CNT with hydrocarbons is difficult due to the low reactivity of these systems. Classical molecular dynamics and ab-initio calculations have been employed to study the adsorption improvement of accelerated $\mathrm{CH}_{4}$ molecules (with energy in the range $5-100 \mathrm{eV}$ ) on CNTs [49]. As methane cracking occurs, the obtained radicals $\left(\mathrm{CH}_{3}, \mathrm{CH}_{2}\right.$ and $\left.\mathrm{CH}\right)$ are adsorbed in different ways depending on the incident energy, while no decoration is observed at low energy, $\mathrm{CH}_{4}$ dissociates into carbon (that is adsorbed and the CNT wall) and hydrogen molecules for incident energy higher than $60 \mathrm{eV}$. Collisions can also break the tube wall and form structural defects that can be healed through high temperature annealing $(2000 \mathrm{~K})$, provided the incident energy is lower than $70 \mathrm{eV}$. Among the investigated SWCNT structures, the ones with larger radius show lower reactivity. The adhesion of radicals modifies the SWCNT transport properties as evidenced by the calculated DOS where localized energy state appears in the gap for $\mathrm{CH}_{3}$ and $\mathrm{CH}$ adsorption. Weak binding between $\mathrm{CH}_{4}$ and a $(5,5)$ SWCNT is confirmed at zero temperature [48] while recent tight binding molecular dynamics calculations have evidenced that at room temperature dissociation reaction proceeds with low enthalpy change, provided the thermal energy is sufficient to get the methane and the CNT close enough [52]. 
TABLE 4: A selection of recent calculations concerning gas adsorption on graphene.

\begin{tabular}{|c|c|c|c|c|c|c|}
\hline \multicolumn{2}{|c|}{ Gas type } & \multirow{2}{*}{$\begin{array}{c}\text { Adsorption site } \\
\mathrm{T}\end{array}$} & \multirow{2}{*}{$\begin{array}{c}E_{b}(\mathrm{eV}) \\
-0.235\end{array}$} & \multirow{2}{*}{$\begin{array}{c}d_{0}(\mathrm{~nm}) \\
0.327\end{array}$} & \multirow{2}{*}{$\frac{\text { DFT model }}{\text { LDA }}$} & \multirow{2}{*}{$\begin{array}{c}\text { Reference } \\
51]\end{array}$} \\
\hline $\mathrm{C}_{6} \mathrm{H}_{6}$ & $\mathrm{P}$ & & & & & \\
\hline $\mathrm{H}_{2} \mathrm{O}$ & $\mathrm{P}$ & $\mathrm{H}$ & -0.047 & 0.350 & \multirow{5}{*}{ GGA } & \multirow{5}{*}[156]{} \\
\hline $\mathrm{NH}_{3}$ & $\mathrm{P}$ & $\mathrm{H}$ & -0.031 & 0.381 & & \\
\hline $\mathrm{CO}$ & $\mathrm{P}$ & $\mathrm{B} / \mathrm{H}$ & -0.014 & 0.374 & & \\
\hline $\mathrm{NO}_{2}$ & $\mathrm{C} / \mathrm{P}$ & B & -0.067 & 0.361 & & \\
\hline NO & $\mathrm{P}$ & $\mathrm{B}$ & -0.029 & 0.376 & & \\
\hline $\mathrm{NO}_{2}$ & $\mathrm{P}$ & \multirow{2}{*}{ - } & -0.085 & 0.340 & \multirow{2}{*}{ GGA } & \multirow{2}{*}[15]{} \\
\hline $\mathrm{N}_{2} \mathrm{O}_{4}$ & $\mathrm{P}$ & & -0.067 & 0.390 & & \\
\hline $\mathrm{CO}$ & \multirow{7}{*}{$\mathrm{C}$} & \multirow{7}{*}{ dangling bonds } & -1.34 & 0.135 & \multirow{7}{*}{ GGA } & \multirow{7}{*}[170]{} \\
\hline $\mathrm{NO}$ & & & -2.29 & 0.143 & & \\
\hline $\mathrm{NO}_{2}$ & & & -2.70 & 0.148 & & \\
\hline $\mathrm{O}_{2}$ & & & -1.88 & 0.137 & & \\
\hline $\mathrm{N}_{2}$ & & & +0.24 & - & & \\
\hline $\mathrm{CO}_{2}$ & & & -0.31 & 0.150 & & \\
\hline $\mathrm{NH}_{3}$ & & & -0.18 & 0.149 & & \\
\hline $\mathrm{O}_{2}$ & \multirow{4}{*}{$\mathrm{C}$} & \multirow{4}{*}{ divacancy } & -8.44 & & \multirow{4}{*}{ GGA } & \multirow{4}{*}[14]{} \\
\hline $\mathrm{N}_{2}$ & & & -4.53 & - & & \\
\hline $\mathrm{B}_{2}$ & & & -13.83 & - & & \\
\hline $\mathrm{CO}$ & & & -3.86 & & & \\
\hline $\mathrm{F}_{2}$ & \multirow{4}{*}{$\mathrm{P}$} & \multirow{4}{*}{ B } & -0.231 & 0.317 & \multirow{4}{*}{$\begin{array}{l}\text { vdW-DFT } \\
\text { GGA }\end{array}$} & \multirow{4}{*}[160]{} \\
\hline $\mathrm{Cl}_{2}$ & & & -0.259 & 0.323 & & \\
\hline $\mathrm{Br}_{2}$ & & & -0.296 & 0.374 & & \\
\hline $\mathrm{I}_{2}$ & & & 0.523 & 0.380 & & \\
\hline
\end{tabular}

In the "Gas type" column the adsorbed gaseous species and the adsorption phenomena encountered; C and P stand for chemisorption and physisorption, respectively. In the second column. T, H, and B stand for Top, Hollow, and Bridge site, respectively. Blank spaces in the third/fourth columns indicate the missing of the corresponding values in the original paper. Further details are referred in the cited articles.

Doping with metallic particles can enhance the homolytic dissociation of the $\mathrm{H}-\mathrm{CH}_{3}$ bond; indeed, recent DFT calculations [171] have suggested that a zigzag nanotube, decorated with an interstitial $\mathrm{C}$ and Mo, can decrease the energy barrier for $\mathrm{CH}_{4}$ dissociation thanks to the cooperation between the dipole induced in the CNT by the selfinterstitial $\mathrm{C}$ atom and the Mo- $d$-orbitals.

Also simple alkene $\left(\mathrm{C}_{2} \mathrm{H}_{4}\right)$ and alkyne $\left(\mathrm{C}_{2} \mathrm{H}_{2}\right)$ have been proposed for catalytic hydrogenation on Pt-doped armchair nanotube and studied by DFT [44]. The ethylene interaction with a CNT is relatively weak, despite the significant charge transfer in the case of doped SWCNT. In the acetylene case, instead, the interaction is stronger and is presumably related to the observed hybridization transition from $\mathrm{sp}$ to $\mathrm{sp}^{2}$. Metallic doping is not the unique way to improve the CNT reactivity at $0 \mathrm{~K}$; indeed, accurate GGA-DFT calculation performed with a localized basis set (B3LYP/6-311+G* level of theory) have evidenced that nitrogen doping of CNTs enhances the oxygen stability at the CNT sidewall; this circumstance favors the methane cracking at the oxygen impurity at $0 \mathrm{~K}$ through the orbitals overlap [172]. Then nitrogen doped CNTs can be engineered in order to obtain highly reactive catalysts, comparable to metal ones.
Besides it is only marginally pertaining the theme of gas-CNT interaction, it is worth to mention that CNTs can be functionalized to improve their solubility in water or in organic solvents that is important for, for example, nanomedicine. For instance, the functionalization of a CNT with a carboxylic group or methane-derived radicals $\left(-\mathrm{CH}_{2}-\mathrm{OH},-\mathrm{CH}_{2}-\mathrm{Cl},-\mathrm{CH}_{2}-\mathrm{SH}\right.$, and $\left.-\mathrm{CONH}-\mathrm{CH}_{3}\right)$, has been investigated with regard to the $-\mathrm{OH}$ free radical scavenging capability [173]. ab-initio calculations, in the framework of B3LYP hybrid HF-density functional and the $3-311+G(d)$ localized basis set, have shown that the CNT elicity affects the free radical scavenger capacity, armchair tubes being more effective than zig-zag ones. Moreover it is shown that functional groups with the best performance are the ones containing just carbon, hydrogen and nitrogen atoms. Moreover different vacancy defects affect differently the $\mathrm{OH}$ addition on the SWCNT while the Stone-Wales point defects show the largest site dependent effect [174].

The chemical reactivity of CNT for oxygen chemisorption has been addressed by MP2, to obtain accurate binding energy, and DFT calculations (at various levels of theory) for larger system. It is shown that singlet $\mathrm{O}_{2}$ is the most stable chemisorption configuration but it is not expected to occur 

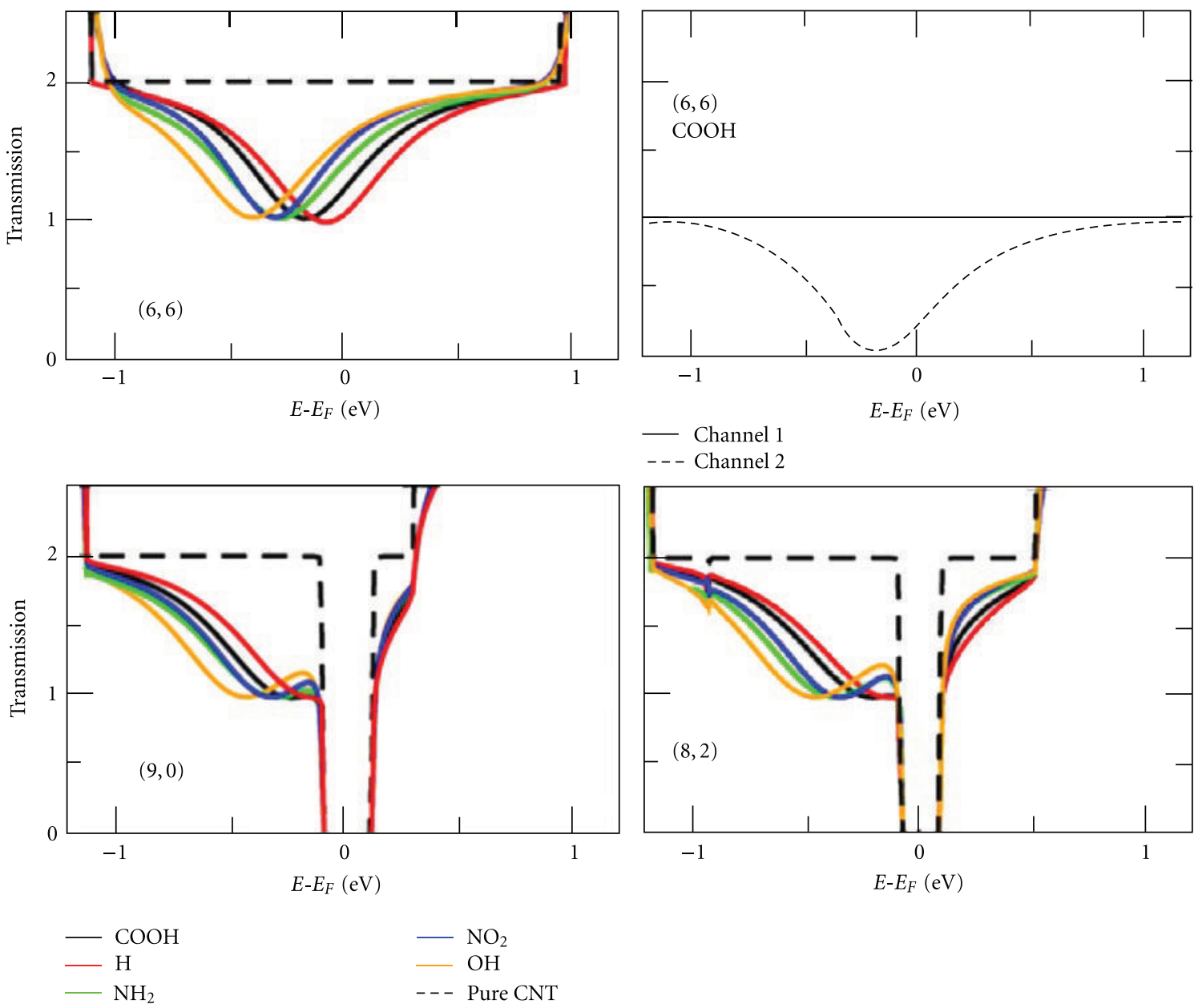

$-\mathrm{H}$

- - - Pure CNT

Figure 25: Top-left: Transmission curves for a $(6,6)$ SWNT with one chemisorbed molecule; top-right: transmission of the two channels in a $(6,6)$ CNT with one $\mathrm{COOH}$ molecule adsorbed. Same transmission curves for $(9,0)$ (bottom-left) and $(8,2)$ (bottom-right) CNTs. From [56]; copyright 2008 by the American Physical Society.

at RT due to the large activation barrier [158]. It should be emphasized that the exchange-correlation functional adopted affect much the $\mathrm{O}_{2}$ ground state properties found within DFT [157].

Using analogous theoretical schemes, $\mathrm{NH}_{3}$ on $(9,0) \mathrm{CNTs}$ has been studied evidencing no charge transfer and suggesting that no chemisorption occurs [175].

CNTs as chemical sensors of other gaseous species can take advantage from the change of the electrical conductivity induced by adsorption of functional groups.

Despite recent controversial data [176], theoretical results seem to indicate that donor or acceptor species may change the carrier density of a $p$-type semiconducting CNT. However, in the case of a metallic CNT, transport properties show a peculiar dependence on the positions of the adsorbed molecules with the possible suppression of conductivity [56]. It is known that transport in a metallic CNT occurs through two channels corresponding to the Bloch states at the $\mathrm{K}$ and $K^{\prime}$ points of the graphene first Brillouin zone. A simple "tight-binding" picture of the coupling between an impurity level $\varepsilon_{0}$ and CNT $p_{z}$-orbitals show that, in the case of an isolated impurity, one of the two channels is suppressed. Accurate DFT and nonequilibrium Green function (NEGF) transport calculations confirm this simple view as shown by the transmission curves for different adsorbates, such as $\mathrm{H}, \mathrm{COOH}, \mathrm{OH}, \mathrm{NH}_{2}$, and $\mathrm{NO}_{2}$, reported in Figure 25. If two impurities are adsorbed on the CNT sidewall, tight binding and DFT-NEGF calculations still agree showing that the transport behavior depends on the relative positions of the two impurities $\Delta \mathbf{R}=n \mathbf{a}_{1}+m \mathbf{a}_{2}$ (being $\mathbf{a}_{1}, \mathbf{a}_{2}$ the basis vectors of graphene): if $n-m=3 p$ for all $p \in Z$ transmission is the same as the one obtained with only one impurity. If the previous condition is not satisfied, the transmission is completely suppressed (see Figure 26). Some molecular species, such as CO, are not chemisorbed on semiconducting SWCNTs; however the local chemical activity can be changed by applying an uniaxial stress orthogonal to the tube axis so that, for example, the $\mathrm{CO}$ molecule can be bonded on the surface [53].

Impurity inclusion in CNTs can improve the chemical sensing of molecules that are not chemisorbed onto pure CNT side wall. ab-initio calculations of a B-doped CNT 


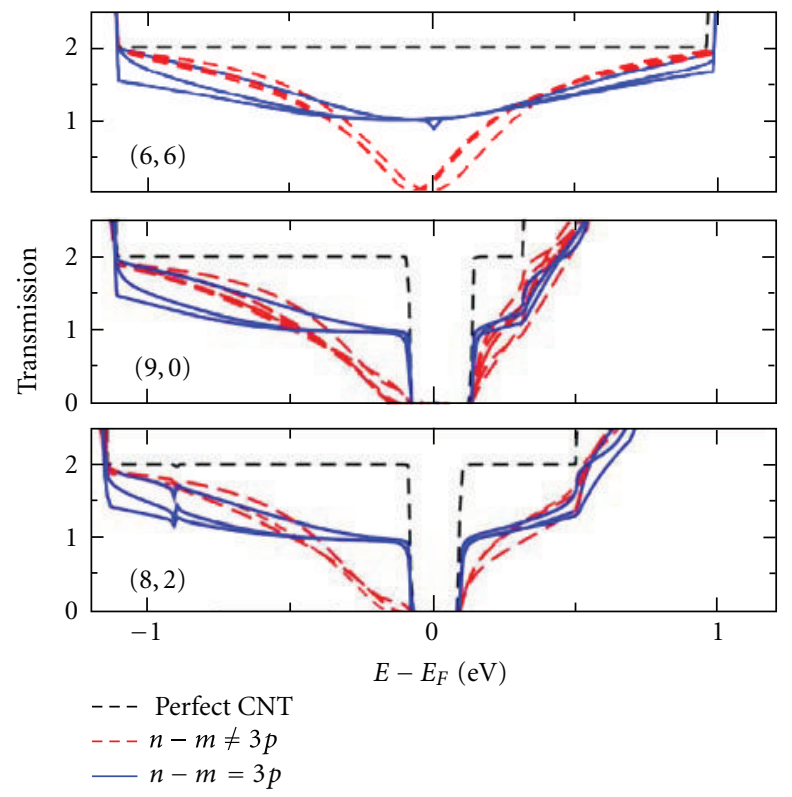

FIgURE 26: Transmission functions of various tubes with two hydrogen atoms adsorbed. The blue (red) solid lines are obtained with $n-m=$ $3 p(n-m \neq 3 p)$. The black dashed line is the transmission function of a pure CNT. From [56]; copyright 2008 by the American Physical Society.

for $\mathrm{CO}$ and $\mathrm{H}_{2} \mathrm{O}$ detection have evidenced an enhanced chemical reactivity with increased binding energy [54] accompanied by a large charge transfer from the nanotube to the molecule.

Systematic investigations on small molecules adsorbed on a Pt doped armchair SWCNT [44] have shown chemisorption and significant charge transfer from the nanotube to the adsorbate for most of the examined species resulting in a change of the CNT conductance. $\mathrm{NH}_{3}$ behaves in the opposite way due to the high LUMO energy of this molecule that, differently from the other cases, inhibits any "backdonation" from the nanotube.

TM-doped CNT structures, perhaps, are the most promising candidates for detection of small molecules under standard conditions. In recent experiments, CNT samples have been pretreated by irradiation with Ar ion beams to form vacancies where TM atoms are strongly bonded thanks to their partially occupied $d$-orbitals.

DFT total energy calculations have shown that substitutional atoms of most of the $3 d$ transition metals ( Ti, V, Cr, $\mathrm{Mn}, \mathrm{Fe}, \mathrm{Co}, \mathrm{Ni}$ ) exhibit a high binding energy in different sites of an armchair carbon nanotube (see Figure 27), with the exception of $\mathrm{Cu}$ and $\mathrm{Zn}$ that are rather unstable because of their fully occupied $d$ bands [55]. The general trend emerging is that light transition metals can bind several adsorbate molecular species $\left(\mathrm{N}_{2}, \mathrm{O}_{2}, \mathrm{H}_{2} \mathrm{O}, \mathrm{CO}, \mathrm{NH}_{3}\right.$, and $\mathrm{H}_{2} \mathrm{~S}$ ) with large binding energy values. Water molecules are weakly bound to most of the active site, suggesting that these sensors are robust against humidity. Ni-doped CNT systems seem to be the most promising candidate for $\mathrm{CO}$ detection as indicated by the conductance data reported, together with the adsorption energies, in Figure 28: indeed for this system we have an electrical resistance change per active site greater than $1 \Omega$. In the same figures, data concerning adsorption of molecular species in mono- and divacancies on CNTs are also reported. In order to give a general view on gas sensing in CNTs, we have chosen to summarize some of the most important results previously discussed in Table 5 where the main features concerning both the binding energy and the ground state configuration distances are provided together with the indication of the level of theory used. For completeness, in the same Table are also reported the data concerning physisorption in various CNT structures.

\section{Conclusions}

The wide variety of data on atomistic simulations of gas adsorption in CNSs, sometimes affected by dispersion, makes it difficult drawing a general scenario. It must be evidenced, however, that "in-silico" experiments play a fundamental role with increasing importance in the understanding of the phenomena involved in adsorption. Besides being a fundamental support to experiments, atomistic simulations and total energy calculations may reveal unexpected phenomena that could lead experimentalists. For instance, while a general agreement has been reached concerning the unsuitability of pure CNSs for hydrogen storage, many predictions on alkaly or transition metal doping indicate a new promising route where, however, problems of contaminants may represent a challenge.

Concerning methane adsorption in CNSs, instead, atomistic simulations predict storage properties close to needs for industrial applications.

$a b$-initio total energy modelling is mandatory for impurities, doping, chemisorption, and sensing due to the inherent complexity of the processes involved. In these cases, 
TABLE 5: A selection of recent calculations regarding gas adsorption on a SWCNT.

\begin{tabular}{|c|c|c|c|c|c|c|c|c|}
\hline \multicolumn{2}{|l|}{ Gas type } & \multicolumn{2}{|c|}{ CNT/dopant } & \multirow{2}{*}{$\frac{\text { Site }}{\mathrm{T}}$} & \multirow{2}{*}{$\frac{E_{b}(\mathrm{eV})}{-0.797}$} & \multirow{2}{*}{$\frac{d_{0}(\mathrm{~nm})}{0.193}$} & \multirow[t]{2}{*}{ DFT model } & \multirow[t]{2}{*}{ Reference } \\
\hline $\mathrm{NO}_{2}$ & $\mathrm{C} / \mathrm{P}$ & $(10,0)$ & - & & & & & \\
\hline $\mathrm{O}_{2}$ & $\mathrm{C} / \mathrm{P}$ & $(10,0)$ & - & $\mathrm{B}$ & -0.509 & 0.232 & \multirow{7}{*}{ LDA } & \multirow{7}{*}[48]{} \\
\hline $\mathrm{H}_{2} \mathrm{O}$ & \multirow{6}{*}{$\mathrm{P}$} & $(10,0)$ & - & $\mathrm{T}$ & -0.143 & 0.143 & & \\
\hline $\mathrm{NH}_{3}$ & & $(5,5)$ & - & $\mathrm{T}$ & -0.162 & 0.299 & & \\
\hline $\mathrm{CH}_{4}$ & & $(10,0)$ & - & $\mathrm{H}$ & -0.190 & 0.317 & & \\
\hline $\mathrm{CO}_{2}$ & & $(5,5)$ & - & $\mathrm{H}$ & -0.109 & 0.354 & & \\
\hline $\mathrm{N}_{2}$ & & $(10,0)$ & - & $\mathrm{H}$ & -0.164 & 0.323 & & \\
\hline $\mathrm{Ar}$ & & $(5,5)$ & - & $\mathrm{H}$ & -0.082 & 0.358 & & \\
\hline $\mathrm{H}_{2} \mathrm{O}$ & \multirow{4}{*}{$\mathrm{P}$} & \multirow{4}{*}{$(8,0)$} & $\mathrm{B}$ & \multirow{4}{*}{-} & -0.560 & 0.170 & \multirow{4}{*}{ LDA } & \\
\hline $\mathrm{H}_{2} \mathrm{O}$ & & & $\mathrm{N}$ & & -0.230 & 0.312 & & \\
\hline $\mathrm{CO}$ & & & B & & -0.850 & 0.152 & & [54] \\
\hline $\mathrm{CO}$ & & & $\mathrm{N}$ & & -0.220 & 0.299 & & {$[54]$} \\
\hline \multirow{2}{*}{$\mathrm{CO}$} & $\mathrm{P}$ & \multirow{2}{*}{$(8,0)$} & \multirow{2}{*}{-} & $\mathrm{H}$ & - & 0.322 & \multirow{2}{*}{ GGA } & \multirow{2}{*}[53]{} \\
\hline & $\mathrm{C}$ & & & H-deformed & -0.510 & 0.185 & & \\
\hline $\mathrm{C}_{6} \mathrm{H}_{6}$ & $\mathrm{P}$ & $(17,0)$ & - & $\mathrm{B}$ & -0.204 & 0.322 & LDA & {$[50]$} \\
\hline $\mathrm{C}_{6} \mathrm{H}_{6}$ & \multirow{4}{*}{$\mathrm{P}$} & \multirow{4}{*}{$(10,0)$} & - & \multirow{4}{*}{ B } & -0.200 & \multirow{4}{*}{0.320} & & \\
\hline $\mathrm{C}_{8} \mathrm{~N}_{2} \mathrm{O}_{2} \mathrm{Cl}_{2}$ & & & - & & -0.610 & & LDA & \\
\hline $\mathrm{C}_{10} \mathrm{H}_{8}$ & & & - & & -0.260 & & LDA & {$[51]$} \\
\hline $\mathrm{C}_{16} \mathrm{H}_{10}$ & & & - & & -0.420 & & & [J1] \\
\hline $\mathrm{C}_{6} \mathrm{H}_{6}$ & & & - & B & -0.260 & 0.312 & & \\
\hline $\mathrm{C}_{6} \mathrm{H}_{5} \mathrm{NH}_{2}$ & & & - & B & -0.302 & 0.286 & & \\
\hline $\mathrm{C}_{6} \mathrm{H}_{5} \mathrm{CH}_{3}$ & & & - & B & -0.264 & 0.277 & & \\
\hline $\mathrm{C}_{6} \mathrm{H}_{5} \mathrm{NO}_{2}$ & & & - & $\mathrm{T}$ & -0.277 & 0.300 & & \\
\hline $\mathrm{NH}_{3}$ & $\mathrm{P}$ & $(8,0)$ & - & $\mathrm{H}$ & -0.113 & 0.271 & LDA & \\
\hline $\mathrm{CH}_{4}$ & & $(0,0)$ & - & $\mathrm{H}$ & -0.095 & 0.279 & LDA & [161] \\
\hline $\mathrm{CH}_{3} \mathrm{NO}_{2}$ & & & - & $\mathrm{H}$ & -0.129 & 0.288 & & \\
\hline $\mathrm{NH}_{2}$ & & & - & $\mathrm{H}$ & -0.152 & 0.239 & & \\
\hline $\mathrm{NO}_{2}$ & & & - & $\mathrm{T}$ & -0.223 & 0.261 & & \\
\hline $\mathrm{CH}_{3}$ & $\mathrm{C}$ & & - & $\mathrm{T}$ & -1.627 & 0.154 & & \\
\hline $\mathrm{CO}$ & & & & & -1.788 & 0.197 & & \\
\hline $\mathrm{NO}$ & & & & & -2.449 & 0.191 & & \\
\hline $\mathrm{N}_{2}$ & $\mathrm{C}$ & $(5,5)$ & $\mathrm{Pt}$ & $\mathrm{H}$ & -1.123 & 0.205 & GGA & \\
\hline $\mathrm{C}_{2} \mathrm{H}_{4}$ & & $(3,5)$ & $\mathrm{Pl}$ & $\Pi$ & -1.146 & 0.231 & GGA & {$[44]$} \\
\hline $\mathrm{C}_{2} \mathrm{H}_{2}$ & & & & & -1.360 & 0.132 & & {$[\Psi x]$} \\
\hline $\mathrm{NH}_{3}$ & & & & & -1.378 & 0.225 & & \\
\hline $\mathrm{COOH}$ & $\mathrm{C}$ & & & & & 0.157 & & \\
\hline $\mathrm{NH}_{2}$ & $\mathrm{C}$ & & & & & 0.149 & & \\
\hline $\mathrm{NO}_{2}$ & $\mathrm{C}$ & - & - & $\mathrm{T}$ & - & 0.164 & GGA & \\
\hline $\mathrm{OH}$ & $\mathrm{C}$ & & & & & 0.145 & & {$[56]$} \\
\hline $\mathrm{CO}$ & $\mathrm{P}$ & & & & & 0.255 & & {$[50]$} \\
\hline $\mathrm{CO}_{2}$ & $\mathrm{P}$ & & & & & 0.252 & & \\
\hline $\mathrm{N}_{2}, \mathrm{O}_{2}, \mathrm{H}_{2} \mathrm{O}, \mathrm{CO}, \mathrm{NH}_{3}, \mathrm{H}_{2} \mathrm{~S}$ & $\mathrm{C}$ & $(6,6)$ & - & Vacancy & - & - & GGA & {$[55]$} \\
\hline
\end{tabular}

simulations give encouraging results and evidence new challenges in controlling the CNSs local chemistry for sensing that is still on the way.

Generally speaking, atomistic modelling has shown that TM doping is most probably the right way to engineer the various different CNSs in order to obtain valuable materials for sensing devices. A careful choice of the correct scheme is often mandatory to avoid artifacts; this should be evaluated case by case because even DFT-LDA can be appropriate for selected systems. 


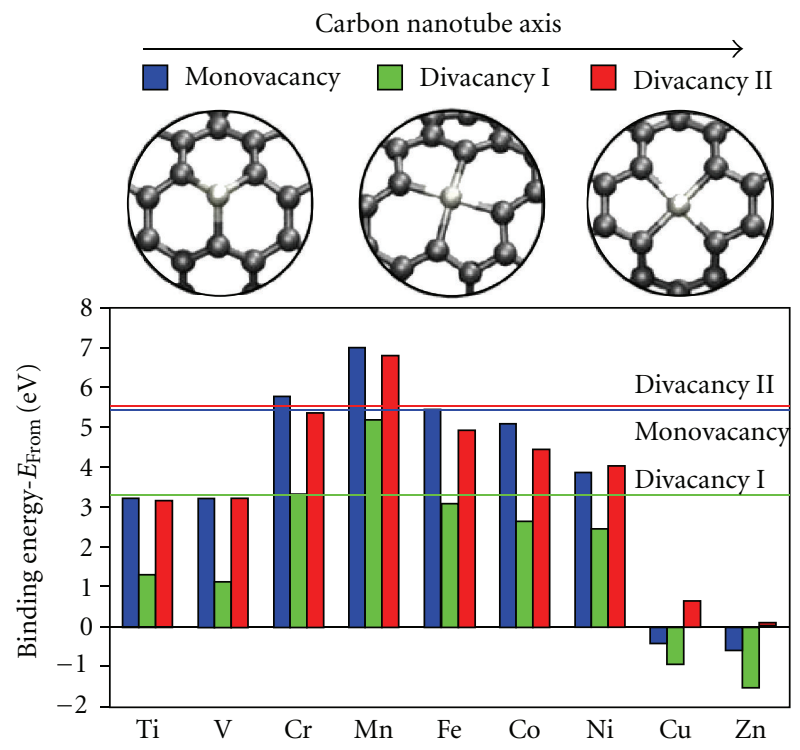

FIGURE 27: Structural schematics and binding energies for a $3 d$ transition metal occupied monovacancy (blue), divacancy I (green) or divacancy II (red) in a $(6,6)$ carbon nanotube. Binding energies of carbon atoms in the same sites are indicated as horizontal lines with the same color code. From [55]; copyright 2010 by the American Physical Society.

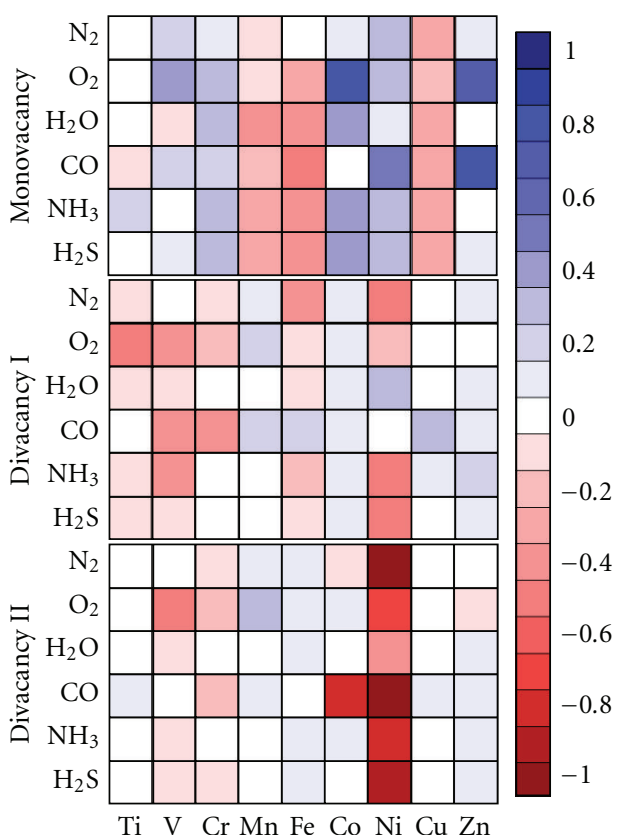

(a)

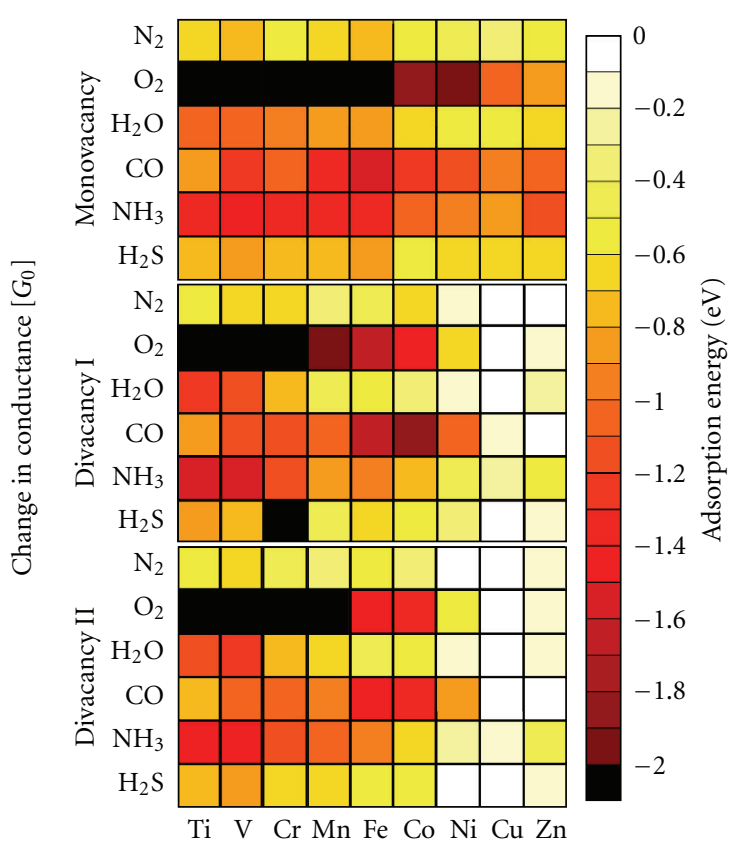

(b)

Figure 28: Calculated change in conductance (a) and adsorption energy (b) for $\mathrm{N}_{2}, \mathrm{O}_{2}, \mathrm{H}_{2} \mathrm{O}, \mathrm{CO}, \mathrm{NH}_{3}$, and $\mathrm{H}_{2} \mathrm{~S}$ on $3 d$ transition metals occupying a monovacancy (top), divacancy I (middle) and divacancy II (bottom) in a $(6,6)$ carbon nanotube. From [56]; copyright 2010 by the American Physical Society.

However it should be emphasized that the relationship between in silico and real experiments is often vitiated by the fact that the most accurate predictions available concern ground state properties; higher temperature values (f.i. even room temperature), in fact, may change dramatically the scenario because most of the carbon nanostructured materials investigated may behave quite differently due to the possible hybridization transition induced by thermal distortions. In the near future, the enormous increase of the computational resources and the improvement of the algorithms should play a key role to make RT in silico experiments, such as accurate ab-initio molecular dynamics, 
achievable also for large systems thus giving a more complete insight to the behavior of real nanostructures for gas adsorption.

\section{References}

[1] S. Satyapal, J. Petrovic, C. Read, G. Thomas, and G. Ordaz, "The U.S. Department of Energy's National Hydrogen Storage Project: progress towards meeting hydrogen-powered vehicle requirements," Catalysis Today, vol. 120, no. 3-4, pp. 246-256, 2007.

[2] E. Bekyarova, M. Davis, T. Burch et al., "Chemically functionalized single-walled carbon nanotubes as ammonia sensors," Journal of Physical Chemistry B, vol. 108, no. 51, pp. 1971719720, 2004.

[3] J. Suehiro, G. Zhou, and M. Hara, "Fabrication of a carbon nanotube-based gas sensor using dielectrophoresis and its application for ammonia detection by impedance spectroscopy," Journal of Physics D, vol. 36, no. 21, pp. L109-L114, 2003.

[4] N. S. Lawrence, R. P. Deo, and J. Wang, "Electrochemical determination of hydrogen sulfide at carbon nanotube modified electrodes," Analytica Chimica Acta, vol. 517, no. 12, pp. 131-137, 2004.

[5] G. E. Froudakis, "Hydrogen interaction with carbon nanotubes: a review of ab initio studies," Journal of Physics Condensed Matter, vol. 14, no. 17, pp. R453-R465, 2002.

[6] D. G. Narehood, J. V. Pearce, P. C. Eklund et al., "Diffusion of $\mathrm{H}_{2}$ adsorbed on single-walled carbon nanotubes," Physical Review B, vol. 67, no. 20, Article ID 205409, 5 pages, 2003.

[7] R. Saito, G. Dressalhaus, and M. S. Dressalhaus, Physical Properties of Carbon Nanotubes, Imperial College Press, London, UK, 1999.

[8] K. S. Novoselov, A. K. Geim, S. V. Morozov et al., "Electric field in atomically thin carbon films," Science, vol. 306, no. 5696, pp. 666-669, 2004.

[9] A. K. Geim and K. S. Novoselov, "The rise of graphene," Nature Materials, vol. 6, no. 3, pp. 183-191, 2007.

[10] F. Cervantes-Sodi, G. Csányi, S. Piscanec, and A. C. Ferrari, "Edge-functionalized and substitutionally doped graphene nanoribbons: electronic and spin properties," Physical Review B, vol. 77, no. 16, Article ID 165427, 13 pages, 2008.

[11] J. S. Arellano, L. M. Molina, A. Rubio, and J. A. Alonso, "Density functional study of adsorption of molecular hydrogen on graphene layers," Journal of Chemical Physics, vol. 112, no. 18, pp. 8114-8119, 2000.

[12] M. H. F. Sluiter and Y. Kawazoe, "Cluster expansion method for adsorption: application to hydrogen chemisorption on graphene," Physical Review B, vol. 68, no. 8, Article ID 085410, 7 pages, 2003.

[13] I. Cabria, M. J. López, and J. A. Alonso, "Enhancement of hydrogen physisorption on graphene and carbon nanotubes by Li doping," Journal of Chemical Physics, vol. 123, no. 20, Article ID 204721, 9 pages, 2005.

[14] B. Sanyal, O. Eriksson, U. Jansson, and H. Grennberg, "Molecular adsorption in graphene with divacancy defects," Physical Review B, vol. 79, Article ID 113409, 4 pages, 2009.

[15] T. O. Wehling, K. S. Novoselov, S. V. Morozov et al., "Molecular doping of graphene," Nano Letters, vol. 8, no. 1, pp. 173-177, 2008.

[16] A. Saffarzadeh, "Modeling of gas adsorption on graphene nanoribbons," Journal of Applied Physics, vol. 107, no. 11, Article ID 114309, 7 pages, 2010.
[17] P. K. Ang, W. Chen, A. T. S. Wee, and P. L. Kian, "Solutiongated epitaxial graphene as $\mathrm{pH}$ sensor," Journal of the American Chemical Society, vol. 130, no. 44, pp. 14392-14393, 2008.

[18] H. Wu, J. Wang, X. Kang et al., "Glucose biosensor based on immobilization of glucose oxidase in platinum nanoparticles/graphene/chitosan nanocomposite film," Talanta, vol. 80, no. 1, pp. 403-406, 2009.

[19] S. M. Gatica, H. I. Li, R. A. Trasca, M. W. Cole, and R. D. Diehl, "Xe adsorption on a $\mathrm{C}_{60}$ monolayer on Ag(111)," Physical Review B, vol. 77, no. 4, Article ID 045414, 8 pages, 2008.

[20] R. A. Trasca, M. W. Cole, T. Coffey, and J. Krim, "Gas adsorption on a $\mathrm{C}_{60}$ monolayer," Physical Review E, vol. 77, no. 4, Article ID 041603, 5 pages, 2008.

[21] R. C. Mowrey, M. M. Ross, and J. H. Callahan, "Molecular dynamics simulations and experimental studies of the formation of endohedral complexes of buckminsterfullerene," Journal of Physical Chemistry, vol. 96, no. 12, pp. 4755-4761, 1992.

[22] M. Yoon, S. Yang, and Z. Zhang, "Interaction between hydrogen molecules and metallofullerenes," Journal of Chemical Physics, vol. 131, no. 6, Article ID 064707, 5 pages, 2009.

[23] Y. H. Kim, Y. Zhao, A. Williamson, M. J. Heben, and S. B. Zhang, "Nondissociative adsorption of $\mathrm{H}_{2}$ molecules in lightelement-doped fullerenes," Physical Review Letters, vol. 96, no. 1, Article ID 016102, 4 pages, 2006.

[24] K. R. S. Chandrakumar and S. K. Ghosh, "Alkali-metalinduced enhancement of hydrogen adsorption in $\mathrm{C}_{60}$ fullerene: an ab initio study," Nano Letters, vol. 8, no. 1, pp. 13-19, 2008.

[25] Q. Sun, P. Jena, Q. Wang, and M. Marquez, "First-principles study of hydrogen storage on $\mathrm{Li}_{12} \mathrm{C}_{60}$," Journal of the American Chemical Society, vol. 128, no. 30, pp. 9741-9745, 2006.

[26] M. Yoon, S. Yang, E. Wang, and Z. Zheng, "Charged fullerenes as high-capacity hydrogen storage media," Nano Letters, vol. 7, no. 9, pp. 2578-2583, 2007.

[27] W. H. Shin, S. H. Yang, W. A. Goddard, and J. K. Kang, "Ni-dispersed fullerenes: hydrogen storage and desorption properties," Applied Physics Letters, vol. 88, no. 5, Article ID 053111, 3 pages, 2006.

[28] T. Yildirim and S. Ciraci, "Titanium-decorated carbon nanotubes as a potential high-capacity hydrogen storage medium," Physical Review Letters, vol. 94, no. 17, Article ID 175501, 4 pages, 2005.

[29] T. Yildirim, J. Íñiguez, and S. Ciraci, "Molecular and dissociative adsorption of multiple hydrogen molecules on transition metal decorated $\mathrm{C}_{60}$," Physical Review B, vol. 72, no. 15, Article ID 153403, 4 pages, 2005.

[30] Y. Zhao, Y. H. Kim, A. C. Dillon, M. J. Heben, and S. B. Zhang, "Hydrogen storage in novel organometallic buckyballs," Physical Review Letters, vol. 94, no. 15, Article ID 155504, 4 pages, 2005.

[31] N. Naghshineh and M. Hashemianzadeh, "First-principles study of hydrogen storage on $\mathrm{Si}$ atoms decorated $\mathrm{C}_{60}$," International Journal of Hydrogen Energy, vol. 34, no. 5, pp. 23192324, 2009.

[32] S. Iijima and T. Ichihashi, "Single-shell carbon nanotubes of 1-nm diameter," Nature, vol. 363, no. 6430, pp. 603-605, 1993.

[33] S. Iijima, "Helical microtubules of graphitic carbon," Nature, vol. 354 , no. 6348 , pp. 56-58, 1991.

[34] F. Darkrim and D. Levesque, "Monte Carlo simulations of hydrogen adsorption in single-walled carbon nanotubes," Journal of Chemical Physics, vol. 109, no. 12, pp. 4981-4984, 1998. 
[35] Y. Ma, Y. Xia, M. Zhao, R. Wang, and L. Mei, "Effective hydrogen storage in single-wall carbon nanotubes," Physical Review $B$, vol. 63, no. 11, Article ID 115422, 6 pages, 2001.

[36] G. Stan and M. W. Cole, "Low coverage adsorption in cylindrical pores," Surface Science, vol. 395, no. 2-3, pp. 280291, 1998.

[37] Q. Wang, J. K. Johnson, and J. Q. Broughton, "Path integral grand canonical Monte Carlo," Journal of Chemical Physics, vol. 107, no. 13, pp. 5108-5117, 1997.

[38] J. S. Arellano, L. M. Molina, A. Rubio, M. J. López, and J. A. Alonso, "Interaction of molecular and atomic hydrogen with $(5,5)$ and $(6,6)$ single-wall carbon nanotubes," Journal of Chemical Physics, vol. 117, no. 5, pp. 2281-2288, 2002.

[39] C. W. Bauschlicher and C. R. So, "High Coverages of Hydrogen on $(10,0),(9,0)$ and $(5,5)$ Carbon Nanotubes," Nano Letters, vol. 2, no. 4, pp. 337-341, 2002.

[40] S. M. Lee and Y. H. Lee, "Hydrogen storage in single-walled carbon nanotubes," Applied Physics Letters, vol. 76, no. 20, pp. 2877-2879, 2000.

[41] X. Zhang, D. Cao, and J. Chen, "Hydrogen adsorption storage on single-walled carbon nanotube arrays by a combination of classical potential and density functional theory," Journal of Physical Chemistry B, vol. 107, no. 21, pp. 4942-4950, 2003.

[42] G. Stan and M. W. Cole, "Hydrogen adsorption in nanotubes," Journal of Low Temperature Physics, vol. 110, no. 1-2, pp. 539-544, 1998.

[43] L. Chen, Y. Zhang, N. Koratkar, P. Jena, and S. K. Nayak, "First-principles study of interaction of molecular hydrogen with Li-doped carbon nanotube peapod structures," Physical Review B, vol. 77, no. 3, Article ID 033405, 4 pages, 2008.

[44] C. S. Yeung, L. V. Liu, and Y. A. Wang, "Adsorption of small gas molecules onto Pt-doped single-walled carbon nanotubes," Journal of Physical Chemistry C, vol. 112, no. 19, pp. 7401-7411, 2008.

[45] H. Xiao, S. H. Li, and J. X. Cao, "First-principles study of Pddecorated carbon nanotube for hydrogen storage," Chemical Physics Letters, vol. 483, no. 1-3, pp. 111-114, 2009.

[46] A. G. Albesa, E. A. Fertitta, and J. L. Vicente, "Comparative study of methane adsorption on single-walled carbon nanotubes," Langmuir, vol. 26, no. 2, pp. 786-795, 2010.

[47] M. M. Calbi, S. M. Gatica, M. J. Bojan, and M. W. Cole, "Phases of neon, xenon, and methane adsorbed on nanotube bundles," Journal of Chemical Physics, vol. 115, no. 21, pp. 9975-9981, 2001.

[48] J. Zhao, A. Buldum, J. Han, and J. P. Lu, "Gas molecule adsorption in carbon nanotubes and nanotube bundles," Nanotechnology, vol. 13, no. 2, pp. 195-200, 2002.

[49] F. Li, Y. Xia, M. Zhao et al., "Selectable functionalization of single-walled carbon nanotubes resulting from $\mathrm{CH}_{n}(n=1-$ 3) adsorption," Physical Review B, vol. 69, no. 16, Article ID 165415, 6 pages, 2004.

[50] F. Tournus and J. C. Charlier, "Ab initio study of benzene adsorption on carbon nanotubes," Physical Review B, vol. 71, no. 16, Article ID 165421, 8 pages, 2005.

[51] F. Tournus, S. Latil, M. I. Heggie, and J. C. Charlier, " $\pi$-stacking interaction between carbon nanotubes and organic molecules," Physical Review B, vol. 72, no. 7, Article ID 075431, 5 pages, 2005.

[52] L. Bagolini, F. Gala, and G. Zollo, "Methane cracking on single-wall carbon nanotubes studied by semi-empirical tight binding simulations," Carbon, vol. 50, no. 2, pp. 411-420, 2012.

[53] L. B. Da Silva, S. B. Fagan, and R. Mota, "Ab initio study of deformed carbon nanotube sensors for carbon monoxide molecules," Nano Letters, vol. 4, no. 1, pp. 65-67, 2004.
[54] S. Peng and K. Cho, "Ab initio study of doped carbon nanotube sensors," Nano Letters, vol. 3, no. 4, pp. 513-517, 2003.

[55] J. M. Garcìa-Lastra, D. J. Mowbray, K. S. Thygesen, A. Rubio, and K. W. Jacobsen, "Modeling nanoscale gas sensors under realistic conditions: computational screening of metal-doped carbon nanotubes," Physical Review B, vol. 81, no. 24, Article ID 245429, 10 pages, 2010.

[56] J. M. Garcìa-Lastra, K. S. Thygesen, M. Strange, and A. Rubio, "Conductance of sidewall-functionalized carbon nanotubes: universal dependence on adsorption sites," Physical Review Letters, vol. 101, no. 23, Article ID 236806, 2008.

[57] J. Jiang and S. I. Sandler, "Nitrogen adsorption on carbon nanotube bundles: role of the external surface," Physical Review B, vol. 68, no. 24, Article ID 245412, 9 pages, 2003.

[58] G. Stan, M. J. Bojan, S. Curtarolo, S. M. Gatica, and M. W. Cole, "Uptake of gases in bundles of carbon nanotubes," Physical Review B, vol. 62, no. 3, pp. 2173-2180, 2000.

[59] F. Fernandez-Alonso, F. J. Bermejo, C. Cabrillo, R. O. Loutfy, V. Leon, and M. L. Saboungi, "Nature of the bound states of molecular hydrogen in carbon nanohorns," Physical Review Letters, vol. 98, no. 21, Article ID 215503, 4 pages, 2007.

[60] H. Tanaka, H. Kanoh, M. El-Merraoui et al., "Quantum effects on hydrogen adsorption in internal nanospaces of single-wall carbon nanohorns," Journal of Physical Chemistry B, vol. 108, no. 45, pp. 17457-17465, 2004.

[61] B. Burteaux, A. Claye, B. W. Smith, M. Monthioux, D. E. Luzzi, and J. E. Fischer, "Abundance of encapsulated $\mathrm{C}_{60}$ in single-wall carbon nanotubes," Chemical Physics Letters, vol. 310, no. 1-2, pp. 21-24, 1999.

[62] A. V. Vakhrushev and M. V. Suetin, "Carbon nanocontainers for gas storage," Nanotechnologies in Russia, vol. 4, no. 11-12, pp. 806-815, 2010.

[63] C. J. Cramer, Essential of Computational Chemistry, John Wiley \& Sons, West Sussex, UK, 2004.

[64] D. Young, Computational Chemistry, John Wiley \& Sons, New York, NY, USA, 2001.

[65] R. O. Jones and O. Gunnarsson, "The density functional formalism, its applications and prospects," Reviews of Modern Physics, vol. 61, no. 3, pp. 689-746, 1989.

[66] E. Engel and R. M. Dreizler, Density Functional Theory-An Advanced Course, Springer, Heidelberg, Germany, 2011.

[67] N. Metropolis, A. W. Rosenbluth, M. N. Rosenbluth, A. H. Teller, and E. Teller, "Equation of state calculations by fast computing machines," The Journal of Chemical Physics, vol. 21, no. 6, pp. 1087-1092, 1953.

[68] D. Frenkel, Understanding Molecular Simulations, Computational Science Series, Academic Press, San Diego, Calif, USA, 2002.

[69] R. Evans, "The nature of the liquid-vapor interface and other topics in the statistical mechanics of non-uniform, classical fluids," Advances in Physics, vol. 28, no. 2, pp. 143-200, 1979.

[70] N. D. Mermin, "Thermal properties of the inhomogeneous electron gas," Physical Review, vol. 137, no. 5, pp. A1441A1443, 1965.

[71] P. Tarazona, "Free-energy density functional for hard spheres," Physical Review A, vol. 31, no. 4, pp. 2672-2679, 1985.

[72] P. Tarazona, U. Marini Bettolo Marconi, and R. Evans, "Phase equilibria of fluid interfaces and confined fluids," Molecular Physics, vol. 60, pp. 573-589, 1987.

[73] P. Hohenberg and W. Kohn, "Inhomogeneous electron gas," Physical Review, vol. 136, no. 3, pp. B864-B871, 1964.

[74] W. Kohn and L. J. Sham, "Self-consistent equations including exchange and correlation effects," Physical Review, vol. 140, no. 4, pp. A1133-A1138, 1965. 
[75] M. C. Payne, M. P. Teter, D. C. Allan, T. A. Arias, and J. D. Joannopoulos, "Iterative minimization techniques for $a b$ initio total-energy calculations: molecular dynamics and conjugate gradients," Reviews of Modern Physics, vol. 64, no. 4, pp. 1045-1097, 1992.

[76] U. Von Barth, "Basic density-functional theory-an overview," Physica Scripta T, vol. T109, pp. 9-39, 2004.

[77] J. E. Dennis and R. B. Schnabel, Numerical Methods for Unconstrained Optimization and Non-Linear Equations, Prentice Hall, Englewood Cliffs, NJ, USA, 1983.

[78] G. B. Bachelet, D. R. Hamann, and M. Schlüter, "Pseudopotentials that work: from $\mathrm{H}$ to Pu," Physical Review B, vol. 26, no. 8, pp. 4199-4228, 1982.

[79] D. R. Hamann, M. Schlüter, and C. Chiang, "Norm-conserving pseudopotentials," Physical Review Letters, vol. 43, no. 20, pp. 1494-1497, 1979.

[80] N. Troullier and J. L. Martins, "Efficient pseudopotentials for plane-wave calculations," Physical Review B, vol. 43, no. 3, pp. 1993-2006, 1991.

[81] D. Vanderbilt, "Soft self-consistent pseudopotentials in a generalized eigenvalue formalism," Physical Review B, vol. 41, no. 11, pp. 7892-7895, 1990.

[82] D. Vanderbilt, "Optimally smooth norm-conserving pseudopotentials," Physical Review B, vol. 32, no. 12, pp. 84128415, 1985.

[83] L. Kleinman and D. M. Bylander, "Efficacious form for model pseudopotentials," Physical Review Letters, vol. 48, no. 20, pp. 1425-1428, 1982.

[84] A. D. Becke, "Density-functional thermochemistry. III. The role of exact exchange," The Journal of Chemical Physics, vol. 98, no. 7, pp. 5648-5652, 1993.

[85] J. P. Perdew, K. Burke, and M. Ernzerhof, "Generalized gradient approximation made simple," Physical Review Letters, vol. 77, no. 18, pp. 3865-3868, 1996.

[86] J. P. Perdew and A. Zunger, "Self-interaction correction to density-functional approximations for many-electron systems," Physical Review B, vol. 23, no. 10, pp. 5048-5079, 1981.

[87] D. M. Ceperley and B. J. Alder, "Ground state of the electron gas by a stochastic method," Physical Review Letters, vol. 45, no. 7, pp. 566-569, 1980.

[88] Y. Okamoto and Y. Miyamoto, "Ab initio investigation of physisorption of molecular hydrogen on planar and curved graphenes," Journal of Physical Chemistry B, vol. 105, no. 17, pp. 3470-3474, 2001.

[89] N. W. Ashcroft and N. D. Mermin, Solid State Physics, Saunders College, Philadelphia, Pa, USA, 1976.

[90] F. Jensen, Introduction to Computational Chemistry, John Wiley \& Sons, New York, NY, USA, 2nd edition, 2007.

[91] M. Marder, Condensed Matter Physics, John Wiley \& Sons, New York, NY, USA, 2000.

[92] T. Helgaker, P. Jorgensen, and J. Olsen, Molecular Electronic Theory, John Wiley \& Sons, New York, NY, USA, 2002.

[93] R. J. Bartlett and M. Musiał, "Coupled-cluster theory in quantum chemistry," Reviews of Modern Physics, vol. 79, no. 1, pp. 291-352, 2007.

[94] G. Stan, V. H. Crespi, M. W. Cole, and M. Boninsegni, "Interstitial He and $\mathrm{Ne}$ in nanotube bundles," Journal of Low Temperature Physics, vol. 113, no. 3-4, pp. 447-452, 1998.

[95] D. M. Ceperley, "Path integrals in the theory of condensed helium," Reviews of Modern Physics, vol. 67, no. 2, pp. 279$355,1995$.

[96] R. P. Feynman and A. R. Hibbs, Quantum Mechanics and Path Integrals, McGraw-Hill, New York, NY, USA.
[97] J. K. Percus and G. J. Yevick, "Analysis of classical statistical mechanics by means of collective coordinates," Physical Review, vol. 110, no. 1, pp. 1-13, 1958.

[98] A. C. Dillon, K. M. Jones, T. A. Bekkedahl, C. H. Kiang, D. S. Bethune, and M. J. Heben, "Storage of hydrogen in singlewalled carbon nanotubes," Nature, vol. 386, no. 6623, pp. 377-379, 1997.

[99] V. Meregalli and M. Parrinello, "Review of theoretical calculations of hydrogen storage in carbon-based materials," Applied Physics A, vol. 72, no. 2, pp. 143-146, 2001.

[100] G. E. Ioannatos and X. E. Verykios, "H2 storage on singleand multi-walled carbon nanotubes," International Journal of Hydrogen Energy, vol. 35, no. 2, pp. 622-628, 2010.

[101] K. L. Lim, H. Kazemian, Z. Yaakob, and W. R. W. Daud, "Solid-state materials and methods for hydrogen storage: a critical review," Chemical Engineering and Technology, vol. 33, no. 2, pp. 213-226, 2010.

[102] C. Liu, Y. Chen, C. Z. Wu, S. T. Xu, and H. M. Cheng, "Hydrogen storage in carbon nanotubes revisited," Carbon, vol. 48, no. 2, pp. 452-455, 2010.

[103] K. A. Williams and P. C. Eklund, "Monte Carlo simulations of $\mathrm{H}_{2}$ physisorption in finite-diameter carbon nanotube ropes," Chemical Physics Letters, vol. 320, no. 3-4, pp. 352-358, 2000.

[104] I. F. Silvera and V. V. Goldman, "The isotropic intermolecular potential for $\mathrm{H}_{2}$ and $\mathrm{D}_{2}$ in the solid and gas phases," The Journal of Chemical Physics, vol. 69, no. 9, pp. 4209-4213, 1978.

[105] A. D. Crowell and J. S. Brown, "Laterally averaged interaction potentials for ${ }^{1} \mathrm{H}_{2}$ and ${ }^{2} \mathrm{H}_{2}$ on the (0001) graphite surface," Surface Science, vol. 123, no. 2-3, pp. 296-304, 1982.

[106] H. Dodziuk and G. Dolgonos, "Molecular modeling study of hydrogen storage in carbon nanotubes," Chemical Physics Letters, vol. 356, no. 1-2, pp. 79-83, 2002.

[107] A. Chambers, C. Park, R. T. K. Baker, and N. M. Rodriguez, "Hydrogen storage in graphite nanofibers," Journal of Physical Chemistry B, vol. 102, no. 22, pp. 4253-4256, 1998.

[108] M. Volpe and F. Cleri, "Role of surface chemistry in hydrogen adsorption in single-wall carbon nanotubes," Chemical Physics Letters, vol. 371, no. 3-4, pp. 476-482, 2003.

[109] S. K. Bhatia and A. L. Myers, "Optimum conditions for adsorptive storage," Langmuir, vol. 22, no. 4, pp. 1688-1700, 2006.

[110] J. Guan, X. Pan, X. Liu, and X. Bao, "Syngas segregation induced by confinement in carbon nanotubes: a combined first-principles and Monte Carlo study," Journal of Physical Chemistry C, vol. 113, no. 52, pp. 21687-21692, 2009.

[111] S. Dag, Y. Ozturk, S. Ciraci, and T. Yildirim, "Adsorption and dissociation of hydrogen molecules on bare and functionalized carbon nanotubes," Physical Review B, vol. 72, no. 15, Article ID 155404, 8 pages, 2005.

[112] T. A. Halgren, "Representation of van der Waals (vdW) interactions in molecular mechanics force fields: potential form, combination rules, and vdW parameters," Journal of the American Chemical Society, vol. 114, no. 20, pp. 7827-7843, 1992.

[113] B. Kuchta, L. Firlej, P. Pfeifer, and C. Wexler, "Numerical estimation of hydrogen storage limits in carbon-based nanospaces," Carbon, vol. 48, no. 1, pp. 223-231, 2010.

[114] J. Cheng, X. Yuan, X. Fang, and L. Zhang, "Computer simulation of hydrogen physisorption in a Li-doped single walled carbon nanotube array," Carbon, vol. 48, no. 2, pp. 567-570, 2010.

[115] H. Lee, J. Ihm, M. L. Cohen, and S. G. Louie, "Calcium-decorated carbon nanotubes for high-capacity hydrogen storage: 
first-principles calculations," Physical Review B, vol. 80, no. 11, Article ID 115412, 5 pages, 2009.

[116] A. Touzik and H. Hermann, "Theoretical study of hydrogen adsorption on graphitic materials," Chemical Physics Letters, vol. 416, no. 1-3, pp. 137-141, 2005.

[117] W. A. Steele and M. J. Bojan, "Simulation studies of sorption in model cylindrical micropores," Advances in Colloid and Interface Science, vol. 76-77, pp. 153-178, 1998.

[118] L. Zhan, K. Li, X. Zhu, C. Lv, and L. Ling, "Adsorption limit of supercritical hydrogen on super-activated carbon," Carbon, vol. 40, no. 3, pp. 455-457, 2002.

[119] M. Georgakis, G. Stavropoulos, and G. P. Sakellaropoulos, "Molecular dynamics study of hydrogen adsorption in carbonaceous microporous materials and the effect of oxygen functional groups," International Journal of Hydrogen Energy, vol. 32, no. 12, pp. 1999-2004, 2007.

[120] T. X. Nguyen, N. Cohaut, J. S. Bae, and S. K. Bhatia, "New method for atomistic modeling of the microstructure of activated carbons using hybrid reverse Monte Carlo simulation,” Langmuir, vol. 24, no. 15, pp. 7912-7922, 2008.

[121] G. Opletal, T. Petersen, B. O’Malley et al., "Hybrid approach for generating realistic amorphous carbon structure using metropolis and reverse Monte Carlo," Molecular Simulation, vol. 28, no. 10-11, pp. 927-938, 2002.

[122] N. Marks, "Modelling diamond-like carbon with the environment-dependent interaction potential," Journal of Physics Condensed Matter, vol. 14, no. 11, pp. 2901-2927, 2002.

[123] D. W. Brenner, O. A. Shenderova, J. A. Harrison, S. J. Stuart, B. Ni, and S. B. Sinnott, "A second-generation reactive empirical bond order (REBO) potential energy expression for hydrocarbons," Journal of Physics Condensed Matter, vol. 14, no. 4, pp. 783-802, 2002.

[124] L. M. Sesè, "Feynman-Hibbs potentials and path integrals for quantum Lennard-Jones systems: theory and Monte Carlo simulations," Molecular Physics, vol. 85, pp. 931-947, 1995.

[125] T. X. Nguyen, J. S. Bae, Y. Wang, and S. K. Bhatia, "On the strength of the hydrogen-carbon interaction as deduced from physisorption," Langmuir, vol. 25, no. 8, pp. 4314-4319, 2009.

[126] D. Levesque, A. Gicquel, F. L. Darkrim, and S. B. Kayiran, "Monte Carlo simulations of hydrogen storage in carbon nanotubes," Journal of Physics Condensed Matter, vol. 14, no. 40, pp. 9285-9293, 2002.

[127] S. J. V. Frankland and D. W. Brenner, "Hydrogen Raman shifts in carbon nanotubes from molecular dynamics simulation," Chemical Physics Letters, vol. 334, no. 1-3, pp. 18-23, 2001.

[128] S. C. Wang, L. Senbetu, and C. W. Woo, "Superlattice of parahydrogen physisorbed on graphite surface," Journal of Low Temperature Physics, vol. 41, no. 5-6, pp. 611-628, 1980.

[129] W. A. Steele, The Interaction of Gases with Solid Surfaces, Pergamon Press, New York, N Y, USA, 1974.

[130] H. Miyaoka, T. Ichikawa, and Y. Kojima, "The reaction process of hydrogen absorption and desorption on the nanocomposite of hydrogenated graphite and lithium hydride," Nanotechnology, vol. 20, no. 20, Article ID 204021, 2009.

[131] P. Guay, B. L. Stansfield, and A. Rochefort, "On the control of carbon nanostructures for hydrogen storage applications," Carbon, vol. 42, no. 11, pp. 2187-2193, 2004.

[132] D. J. Browning, M. L. Gerrard, J. B. Lakeman, I. M. Mellor, R. J. Mortimer, and M. C. Turpin, "Studies into the storage of hydrogen in carbon nanofibers: proposal of a possible reaction mechanism," Nano Letters, vol. 2, no. 3, pp. 201-205, 2002.
[133] O. N. Srivastava and B. K. Gupta, "Further studies on microstructural characterization and hydrogenation behaviour of graphitic nanofibres," International Journal of Hydrogen Energy, vol. 26, no. 8, pp. 857-862, 2001.

[134] Z. H. Zhu, G. Q. Lu, and S. C. Smith, "Comparative study of hydrogen storage in Li- and K-doped carbon materialstheoretically revisited," Carbon, vol. 42, no. 12-13, pp. 25092514, 2004.

[135] H. Lee, J. Ihm, M. L. Cohen, and S. G. Louie, "Calciumdecorated graphene-based nanostructures for hydrogen storage," Nano Letters, vol. 10, no. 3, pp. 793-798, 2010.

[136] H. An, C. S. Liu, Z. Zeng, C. Fan, and X. Ju, "Li-doped B2 C graphene as potential hydrogen storage medium," Applied Physics Letters, vol. 98, no. 17, Article ID 173101, 3 pages, 2011.

[137] M. Andersen, L. Hornekær, and B. Hammer, "Graphene on metal surfaces and its hydrogen adsorption: a meta-GGA functional study," Physical Review B, vol. 86, no. 8, Article ID 085405, 6 pages, 2012.

[138] A. Sigal, M. I. Rojas, and E. P. M. Leiva, "Is hydrogen storage possible in metal-doped graphite 2D systems in conditions found on earth?" Physical Review Letters, vol. 107, no. 15, Article ID 158701, 4 pages, 2011.

[139] G. J. Kubas, "Metal-dihydrogen and $\sigma$-bond coordination: the consummate extension of the Dewar-Chatt-Duncanson model for metal-olefin $\pi$ bonding," Journal of Organometallic Chemistry, vol. 635, no. 1-2, pp. 37-68, 2001.

[140] Q. Sun, Q. Wang, P. Jena, and Y. Kawazoe, "Clustering of Ti on a $\mathrm{C}_{60}$ surface and its effect on hydrogen storage," Journal of the American Chemical Society, vol. 127, no. 42, pp. 1458214583, 2005.

[141] M. Yoon, S. Yang, C. Hicke, E. Wang, D. Geohegan, and Z. Zhang, "Calcium as the superior coating metal in functionalization of carbon fullerenes for high-capacity hydrogen storage," Physical Review Letters, vol. 100, no. 20, Article ID 206806, 4 pages, 2008.

[142] A. J. Maeland and A. T. Skjeltrop, Inventors; Hydrogen storage in carbon material. patent 6290753. 2001.

[143] A. Gotzias, H. Heiberg-Andersen, M. Kainourgiakis, and T. Steriotis, "Grand canonical Monte Carlo simulations of hydrogen adsorption in carbon cones," Applied Surface Science, vol. 256, no. 17, pp. 5226-5231, 2010.

[144] P. B. Sorokin, H. Lee, L. Y. Antipina, A. K. Singh, and B. I. Yakobson, "Calcium-decorated carbyne networks as hydrogen storage media," Nano Letters, vol. 11, no. 7, pp. 26602665, 2011.

[145] D. Cao, X. Zhang, J. Chen, W. Wang, and J. Yun, "Optimization of single-walled carbon nanotube arrays for methane storage at room temperature," Journal of Physical Chemistry $B$, vol. 107, no. 48, pp. 13286-13292, 2003.

[146] W. A. Steele, "The physical interaction of gases with crystalline solids. I. Gas-solid energies and properties of isolated adsorbed atoms," Surface Science, vol. 36, no. 1, pp. 317-352, 1973.

[147] P. Kowalczyk, L. Solarz, D. D. Do, A. Samborski, and J. M. D. MacElroy, "Nanoscale tubular vessels for storage of methane at ambient temperatures," Langmuir, vol. 22, no. 21, pp. 9035-9040, 2006.

[148] F. J. A. L. Cruz and J. P. B. Mota, "Thermodynamics of adsorption of light alkanes and alkenes in single-walled carbon nanotube bundles," Physical Review B, vol. 79, no. 16, Article ID 165426, 14 pages, 2009.

[149] P. Kowalczyk and S. K. Bhatia, "Optimization of slitlike carbon nanopores for storage of hythane fuel at ambient 
temperatures," Journal of Physical Chemistry B, vol. 110, no. 47, pp. 23770-23776, 2006.

[150] P. Kowalczyk, L. Brualla, A. Zywociński, and S. K. Bhatia, "Single-walled carbon nanotubes: efficient nanomaterials for separation and on-board vehicle storage of hydrogen and methane mixture at room temperature?" Journal of Physical Chemistry C, vol. 111, no. 13, pp. 5250-5257, 2007.

[151] X. Peng, D. Cao, and W. Wang, "Heterogeneity characterization of ordered mesoporous carbon adsorbent CMK-1 for methane and hydrogen storage: GCMC simulation and comparison with experiment," Journal of Physical Chemistry C, vol. 112, no. 33, pp. 13024-13036, 2008.

[152] R. J. Dombrowski, D. R. Hyduke, and C. M. Lastoskie, "Pore size analysis of activated carbons from argon and nitrogen porosimetry using density functional theory," Langmuir, vol. 16, no. 11, pp. 5041-5050, 2000.

[153] J. P. Olivier, "Improving the models used for calculating the size distribution of micropore volume of activated carbons from adsorption data," Carbon, vol. 36, no. 10, pp. 14691472, 1998.

[154] P. I. Ravikovitch, A. Vishnyakov, R. Russo, and A. V. Neimark, "Unified approach to pore size characterization of microporous carbonaceous materials from $\mathrm{N}_{2}, \mathrm{Ar}$, and $\mathrm{CO}_{2}$ adsorption isotherms," Langmuir, vol. 16, no. 5, pp. 2311-2320, 2000.

[155] M. B. Sweatman, N. Quirke, W. Zhu, and F. Kapteijn, “Analysis of gas adsorption in Kureha active carbon based on the slit-pore model and Monte-Carlo simulations," Molecular Simulation, vol. 32, no. 7, pp. 513-522, 2006.

[156] O. Leenaerts, B. Partoens, and F. M. Peeters, "Adsorption of $\mathrm{H}_{2} \mathrm{O}, \mathrm{NH}_{2}, \mathrm{CO}, \mathrm{NO}_{2}$, and $\mathrm{NO}$ on graphene: a first-principles study," Physical Review B, vol. 77, no. 12, Article ID 125416, 6 pages, 2008.

[157] P. Giannozzi, R. Car, and G. Scoles, "Oxygen adsorption on graphite and nanotubes," Journal of Chemical Physics, vol. 118, no. 3, pp. 1003-1006, 2003.

[158] A. Ricca, C. W. Bauschlicher, and A. Maiti, "Comparison of the reactivity of $\mathrm{O}_{2}$ with a $(10,0)$ and a $(9,0)$ carbon nanotube," Physical Review B, vol. 68, no. 3, Article ID 035433, 7 pages, 2003.

[159] B. C. Wood, S. Y. Bhide, D. Dutta et al., "Methane and carbon dioxide adsorption on edge-functionalized graphene: a comparative DFT study," Journal of Chemical Physics, vol. 137, no. 5, Article ID 054702, 11 pages, 2012.

[160] A. N. Rudenko, F. J. Keil, M. I. Katsnelson, and A. I. Lichtenstein, "Adsorption of diatomic halogen molecules on graphene: a van der Waals density functional study," Physical Review B, vol. 82, no. 3, Article ID 035427, 7 pages, 2010.

[161] L. M. Woods, Ş. C. Bădescu, and T. L. Reinecke, "Adsorption of simple benzene derivatives on carbon nanotubes," Physical Review B, vol. 75, no. 15, Article ID 155415, 9 pages, 2007.

[162] D. W. Boukhvalov, M. I. Katsnelson, and A. I. Lichtenstein, "Hydrogen on graphene: electronic structure, total energy, structural distortions and magnetism from first-principles calculations," Physical Review B, vol. 77, no. 3, Article ID 035427, 7 pages, 2008.

[163] O. V. Yazyev and L. Helm, "Defect-induced magnetism in graphene," Physical Review B, vol. 75, no. 12, Article ID 125408, 5 pages, 2007.

[164] P. Mohn, Magnetism in the Solid State, vol. 134 of Springer Series in Solid State Sciences, Springer, Berlin, Germany, 2003.

[165] O. Maresca, R. J. M. Pellenq, F. Marinelli, and J. Conard, "A search for a strong physisorption site for $\mathrm{H}_{2}$ in Li-doped porous carbons," Journal of Chemical Physics, vol. 121, no. 24, pp. 12548-12558, 2004.
[166] Y. Miura, H. Kasai, W. Diño, H. Nakanishi, and T. Sugimoto, "First principles studies for the dissociative adsorption of $\mathrm{H}_{2}$ on graphene," Journal of Applied Physics, vol. 93, no. 6, pp. 3395-3400, 2003.

[167] S. Casolo, O. M. Løvvik, R. Martinazzo, and G. F. Tantardini, "Understanding adsorption of hydrogen atoms on graphene," Journal of Chemical Physics, vol. 130, no. 5, Article ID 054704, 10 pages, 2009.

[168] H. Lee, J. Li, G. Zhou, W. Duan, G. Kim, and J. Ihm, "Roomtemperature dissociative hydrogen chemisorption on borondoped fullerenes," Physical Review B, vol. 77, no. 23, Article ID 235101, 5 pages, 2008.

[169] E. Rangel, G. Ruiz-Chavarria, L. F. Magana, and J. S. Arellano, "Hydrogen adsorption on N-decorated single wall carbon nanotubes," Physics Letters, Section A, vol. 373, no. 30, pp. 2588-2591, 2009.

[170] B. Huang, Z. Li, Z. Liu et al., "Adsorption of gas molecules on graphene nanoribbons and its implication for nanoscale molecule sensor," Journal of Physical Chemistry C, vol. 112, no. 35, pp. 13442-13446, 2008.

[171] Z. H. Guo, X. H. Yan, and Y. Xiao, "Dissociation of methane on the surface of charged defective carbon nanotubes," Physics Letters, Section A, vol. 374, no. 13-14, pp. 1534-1538, 2010.

[172] X. Hu, Z. Zhou, Q. Lin, Y. Wu, and Z. Zhang, "High reactivity of metal-free nitrogen-doped carbon nanotube for the C-H activation," Chemical Physics Letters, vol. 503, no. 4-6, pp. 287-291, 2011.

[173] A. Martínez, M. Francisco-Marquez, and A. Galano, "Effect of different functional groups on the free radical scavenging capability of single-walled carbon nanotubes," Journal of Physical Chemistry C, vol. 114, no. 35, pp. 14734-14739, 2010.

[174] A. Galano, M. Francisco-Marquez, and A. Martínez, "Influence of point defects on the free-radical scavenging capability of single-walled carbon nanotubes," Journal of Physical Chemistry C, vol. 114, no. 18, pp. 8302-8308, 2010.

[175] C. W. Bauschlicher and A. Ricca, "Binding of $\mathrm{NH}_{3}$ to graphite and to a $(9,0)$ carbon nanotube," Physical Review B, vol. 70, no. 11, Article ID 115409, 6 pages, 2004.

[176] A. Goldoni, L. Petaccia, S. Lizzit, and R. Larciprete, "Sensing gases with carbon nanotubes: a review of the actual situation," Journal of Physics Condensed Matter, vol. 22, no. 1, Article ID 013001, 2010. 

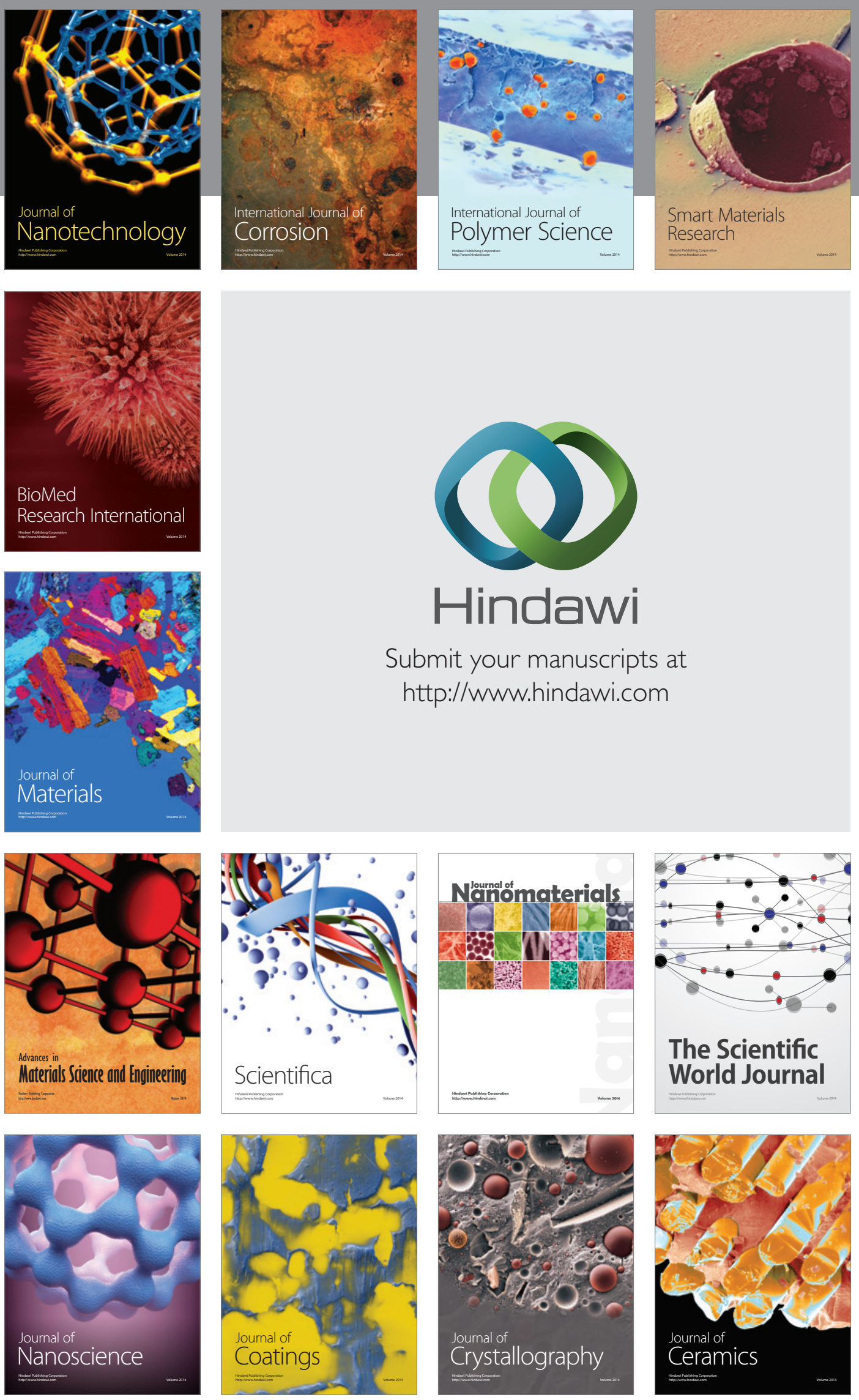

The Scientific World Journal

Submit your manuscripts at

http://www.hindawi.com

\section{World Journal}

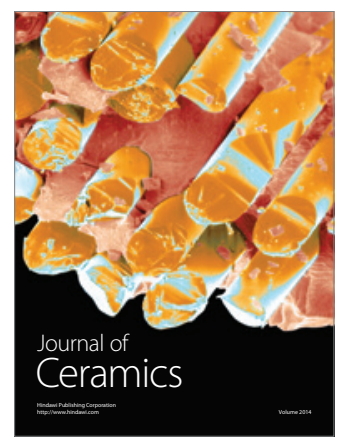

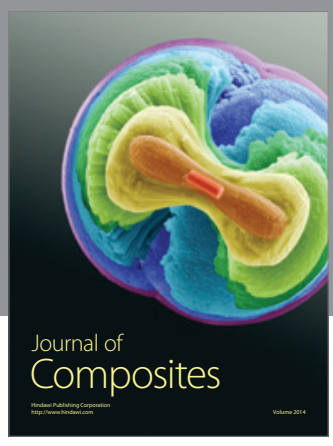
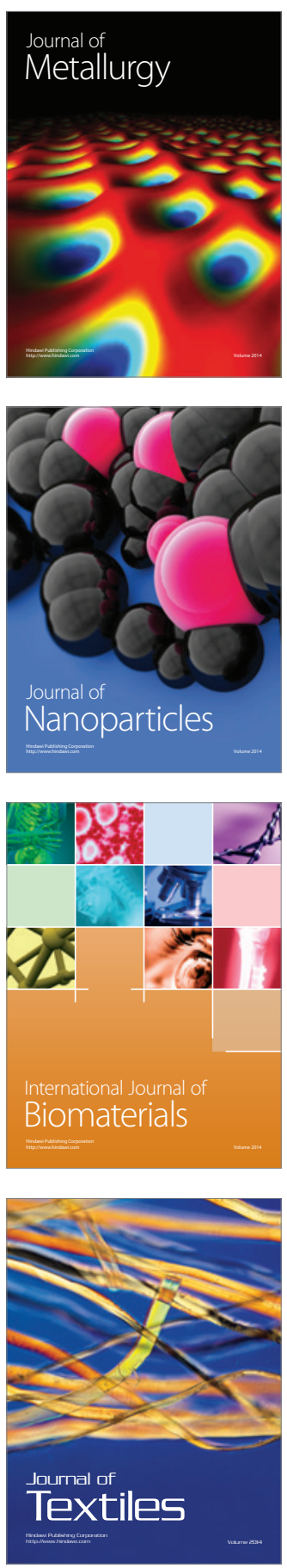